Rowan University

Rowan Digital Works

Theses and Dissertations

$4-24-2014$

\title{
Measuring the efficacy and quality of a professional development training on prejudice reduction and reflective practice: transforming the delivery of change through collective insight and informed action
}

Berdine Gordon-Littrean

Follow this and additional works at: https://rdw.rowan.edu/etd

Part of the Elementary and Middle and Secondary Education Administration Commons

\section{Recommended Citation}

Gordon-Littrean, Berdine, "Measuring the efficacy and quality of a professional development training on prejudice reduction and reflective practice: transforming the delivery of change through collective insight and informed action" (2014). Theses and Dissertations. 528.

https://rdw.rowan.edu/etd/528

This Dissertation is brought to you for free and open access by Rowan Digital Works. It has been accepted for inclusion in Theses and Dissertations by an authorized administrator of Rowan Digital Works. For more information, please contact graduateresearch@rowan.edu. 
MEASURING THE EFFICACY AND QUALITY OF

A PROFESSIONAL DEVELOPMENT TRAINING ON

PREJUDICE REDUCTION AND REFLECTIVE PRACTICE:

TRANSFORMING THE DELIVERY OF CHANGE THROUGH

COLLECTIVE INSIGHT AND INFORMED ACTION

\author{
by \\ Berdine Gordon-Littréan
}

\author{
A Dissertation \\ Submitted to the \\ Department of Educational Leadership \\ College of Education \\ In partial fulfillment of the requirement \\ For the degree of \\ Doctor of Education in Educational Leadership \\ at \\ Rowan University \\ March 13, 2014
}

Dissertation Chair: Yvonne E. González Rodríguez, Ed.D. 
(C) 2014 Berdine Gordon-Littréan 


\section{Dedication}

This manuscript is dedicated to my mother, Cora Belle Gordon, who has supported all my life's endeavors, especially this academic journey to its completion. She always lived her life through her children with the hope that one child would become a 'Doctor.' However, she did not put any specification on what discipline. My mother continuously uplifted me with love and her Godly words of wisdom, instilling in me to "Trust in the LORD with all thine heart; and lean not unto thine own understanding. In all thy ways acknowledge Him, and He shall direct thy paths" (Proverbs 3:5-6, KJV). In addition, I dedicate this manuscript to my late Aunt, Pearl L. Ross (1933-2012), whose untimely departure not only changed the bittersweet normal in my life, but in reflection reminded me to "finish what you start." Knowing my love for dance, Aunt Pearl would also remind me through song that "there may be tear drops to shed. So, while there's moonlight, and music, and love, and romance...Let's face the music and dance!" (Berlin, 1936)

Furthermore, this manuscript is dedicated to my late brother, Ralph G. Gordon (1960-1974), who paved the trail for me, academically, to follow towards my own education journey. Ralph excelled in his early academic and leadership achievements. Although his life shared with us was shorted, I dedicate this Doctor of Education degree to Ralph in my endeavor to carry out his shared dream and educational legacy to its completion. My brother was not only exemplary academically, he was as well a revered community youth leader. It was through the Camden City Public Schools' educators who acknowledged him as a true Servant Leader. My brother was a young change agent in helping to transform the school environment and the hearts of those individuals that were learning to lead by his legacy example set of scholarship, leadership, and service. "To my 
brother Ralph, thank you for watching over me. When I felt alone, especially during those known darkest hours, you were always there in spirit to keep me moving forward, and finish this dissertation to its completion. In spirit I say, "Ralph, as 'we' get conferred this Doctor of Education degree, I shall salute you in the sky above 'to the heavens from whence cometh my help.' Thank you for being my model hero, Ralph."

Finally, one quiet reflection I have in the dedication of this manuscript is that of my mentor and a renowned early childhood educator, the late Wilma Evans Robinson, M.Ed. (1931-2008) who did not complete her dissertation; however, impressed upon me the significance and magnitude of using my brain, creativity, and attaining my degrees. She lives on in my heart and always will. Mrs. Robinson impacted my life at all levels of leadership. Her hope was to witness the completion of my doctoral studies; however, GOD loved her more, and He called her home.

Reflectively, I must give recognition and hold high regarded admiration for the huge leadership influences she has placed on me to complete my doctoral studies. I have known this woman for most of my professional 20 year career in public education administration. Mrs. Robinson exemplified educational leadership as a great servant educational leader. It was no accident that our personal (and, professional later) lives connected early on in my educational leadership path. In addition, I believe that one cannot be an exceptional leader without being a great mentor. She had, through her kind generosity, through her sharing of experiences, and storytelling shaped me in profound ways to be the educational leader I have yet perfected as she so graciously had done in her professional lifetime. 
What I have learned foremost is that mentorship is about teaching, and teaching is the most essential element in leadership. Like all teaching, mentorship is the ability to transfer skills and knowledge. I have benefited greatly from the mentorship of Mrs. Robinson, whose physical and professional presence has shaped early childhood development administration as well as primary and secondary education in this generation, and specifically my own generation. More so, mentorship is the ability to encourage others and inspire them to stretch their capabilities "beyond your wildest dreams!" as Mrs. Robinson would say. Likewise, relationship management is significant where the mentor is inspirational, "guiding and motivating with a compelling vision" (Goleman, 2002, p. 39). Mrs. Robinson was primarily the one individual who observed my academic and professional astuteness as an emerging educational leader. She helped me to identify my leadership traits and management style through self assessment, feedback, and guidance while watching me grow. My EQ learned competencies were gradually discovered to find that using my "gut sense to guide decisions" as she effectively modeled.

In dedication, Mrs. Robinson, may this dissertation impart some insight on what you taught and encouraged: "Do more than belong: participate. Do more than care: help. Do more than believe: practice. Do more than be fair: be kind. Do more than forgive: forget. Do more than dream: work" (William Arthur Ward, 1921-1994). Thank you, Mrs. Robinson, for the positive professional impact you have had on my life to serve, especially the children and families of the early education community. 


\section{Acknowledgements}

My thanks are extended first and foremost to GOD for giving me the foresighted knowledge that "I can do all things through Christ which strengtheneth me" (Philippians $4: 13, \mathrm{KJV})$. I was highly blessed to have many exceptional and wonderful people in my life to help as well as guide me from the initial development stages of my dissertation work until successful completion. These exceptional educational leaders possess "the gift for institutional building [to] create a legacy that will last for a very long time" (Wren, 1995, p.187).

Secondly, I would like to thank my children. Eboné, my $1^{\text {st }}$ born, high school graduate, and future Civil Engineer, thank you for being second mommy to your baby sister while you too were studying to complete your Honors and Advance Placement STEM course studies. Éva, my baby girl, thank you for all your numerous mommy love notes. I especially appreciate hearing your telephone voicemail messages to just say "See you when you get home. I love you, Mommy!” while I was in evening classes and out late nights at the library. Special thanks to your father, Mr. Littréan, for taking the blows and his rigorous transformations to Mr. Mom for you and Eboné when mommy had to disappear for a bit.

Thirdly, I want to express my appreciation to my Doctoral Committee: Dissertation Chair, Dr. Yvonne E. González Rodríguez, as well as Committee Members, Dr. Pamela M. Moore and Dr. Gerard A. Schaller. Thank you all for your extreme patience in the face of numerous obstacles. Many thanks for your support, feedback, and quick responses to my many iterations of this effort. To my chair, Dr. Rodríguez, thank you for your valuable time, patience, understanding, and most importantly believing in my research topic. 
Dr. Moore, one of my committee members, thank you for your infectious enthusiasm and encouragement to keep up the momentum as I progressed through each hurdle faced. Dr. Schaller, thank you for also serving on my committee, and for guiding me through my School Administration internship while simultaneously pursuing the Educational Leadership doctoral program. You taught me what resilience is to an instructional leader as well understanding that "this too shall pass" in regards to the numerous obstacles faced during my School Administration internship and doctoral program. Thank you, Dr. Schaller, for believing in me even before I had complete confidence in my own abilities. Furthermore, "leadership is the ability of a superior to influence the behavior of a subordinate or group and persuade them to follow a particular course of action." I learned this concept during Dr. JoAnn B. Manning's Week 1 of Leadership Theory, my first semester course in the Fall of 2009. Likewise, Nelson Mandela (2003) once said "Education is the most powerful weapon which you can use to change the world." With that being said, the direction for the organization is initially spearheaded by its leader to define a vision. With much gratitude, special thanks to my first employer, the Black People's Unity Movement (BPUM) Impact Corporation and its early education program administration. To Ms. Cheryl A. Randolph, my former supervisor, thank you for hiring me nearly 26 years ago and keeping me under your employ as Consulting Head Teacher. Your well versed familiarity with the organizational needs and ideas of the dissertation project was most helpful during the early programming phase of this undertaking.

Lastly, I am grateful to the following individuals: Africana and Urban Studies specialist (Rutgers University), Brian G. Beckett. You really got me through some very tough times during my dissertation research process experiences. I am hugely grateful for 
your "overtime" work, staying late with me until what I articulated in my head was clear and succinct on paper. To my dissertation seminar course advisors, Dr. James Coaxum, III and Dr. JoAnn B. Manning, thanks for their feedback and initial technical assistance in the early preparation stages of this manuscript. - Thank you for setting the stage! 


\author{
Abstract \\ Berdine Gordon-Littréan \\ MEASURING THE EFFICACY AND QUALITY OF \\ A PROFESSIONAL DEVELOPMENT TRAINING ON \\ PREJUDICE REDUCTION AND REFLECTIVE PRACTICE: \\ TRANSFORMING THE DELIVERY OF CHANGE THROUGH \\ COLLECTIVE INSIGHT AND INFORMED ACTION \\ March 2014 \\ Yvonne E. González Rodríguez, Ed.D. \\ Doctor of Education in Educational Leadership
}

The overall objective of school improvement planning is an enhanced level of student achievement through an enhanced leadership framework and successfully implemented program. The improvement plan process requires focus on specific priorities to effect real change. The purpose of this action research study was to provide professional training as part of a continuum change initiative. The study analyzed the salient factors or areas of critical concern, impacting prejudice and controversial issues settings. These variables included levels of leadership, and significance of the following: diversity of identity and experiences, stereotyping, internalized oppression, building pride and capacity, becoming allies, making commitments to change, as well as appreciation of differences in others. This information obtained by the researcher provided baseline data, which laid the groundwork to develop a school improvement plan.

This study explored the observed impact of the professional development training on agency employees and disposition toward sustained change in prejudice reduction in their applied practice over a period time. Additionally, the action research methodology procedures used for contextualizing and analyzing the efficacy and impact of prejudice reduction in urban education were described. The study's structured conceptual (Brown, 2009; Clark, 2004) and theoretical frameworks (McKown, 2005; Kotter,1996) reflected a 
detailed, yet objective analysis on the social justice issue of prejudice reduction that impacts the school environment, especially impacting the agency's early education program administration and its childhood professionals' psychosocial self awareness.

Data supported the implication that the eight week diversity leadership training and workshop module activities had a positive impact on the sample group. The participants, who responded and shared their stories, affirmed that the professional training received was important to them both personally and professionally. 


\section{Table of Contents}

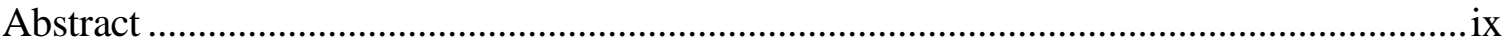

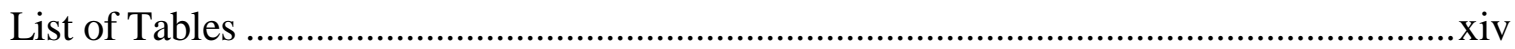

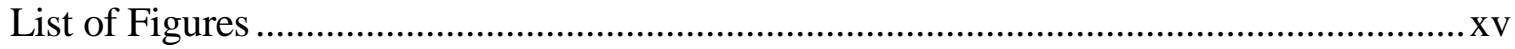

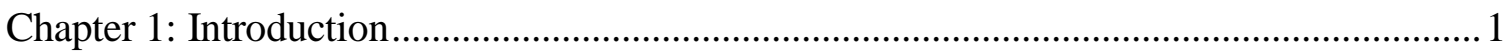

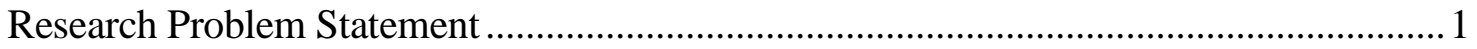

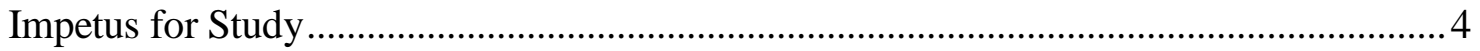

Evaluation and Inquiry Approach as Intervention ........................................................5

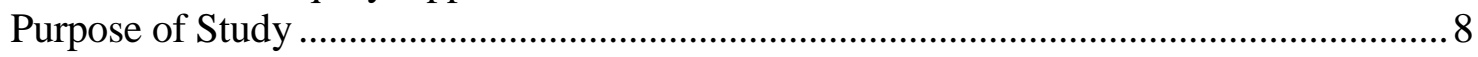

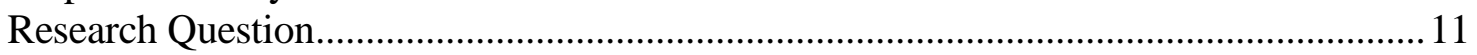

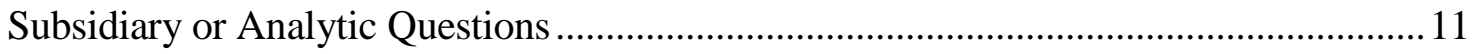

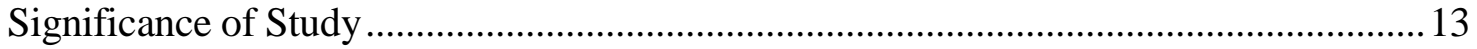

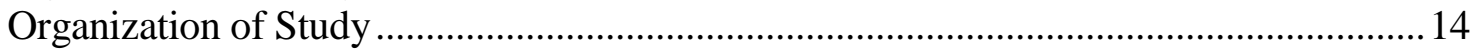

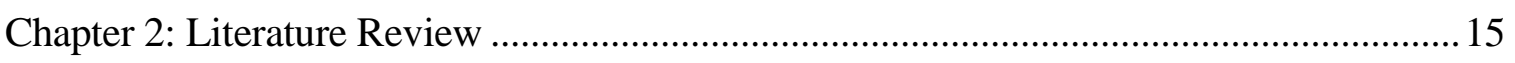

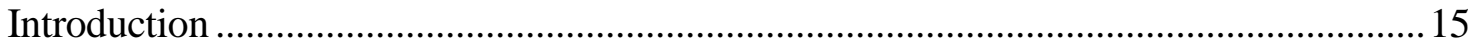

Theoretical Framework: Ecological Assessment ......................................................... 16

Kotter's Theoretical Approach: Eight Stage Process......................................................... 19

Theoretical Framework Model for Action Research .....................................................2

Overview of Action Research Cycles ........................................................................29

Action Research \& Professional Development.............................................................2 29

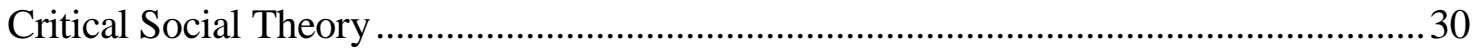

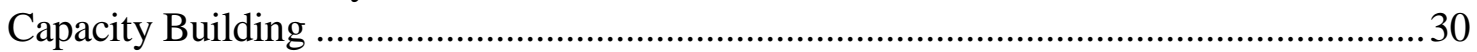

Sociology of the Problem: Models of Cognitive Interaction................................................. 31

Physiology of Problem: A Conceptual Framework ………………………………..........36

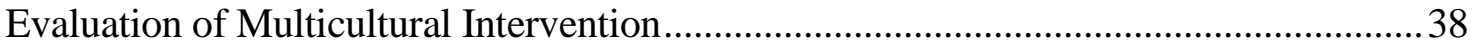

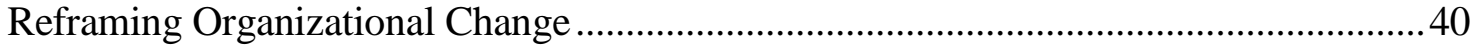

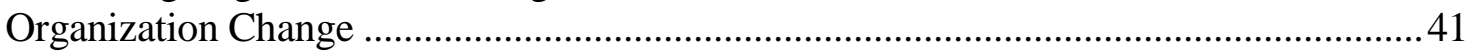

Salient Factors Impacting Prejudice ........................................................................... 41

Leadership for Diversity: A Prejudice Reduction Model ......................................................43

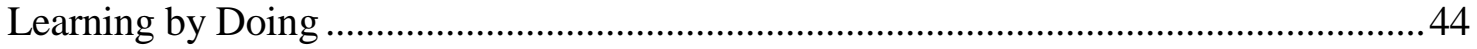

Discussion and Conclusions ……………………………........................................5

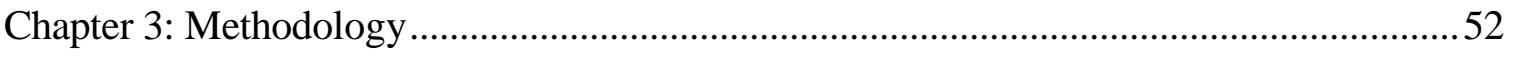

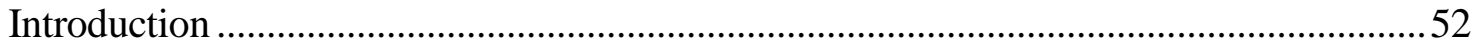

Primary Research Question ...................................................................................53

Subsidiary Analytical Questions ................................................................................53

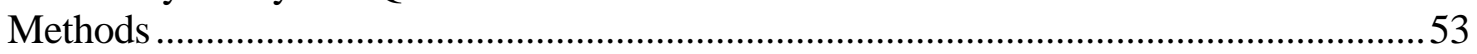

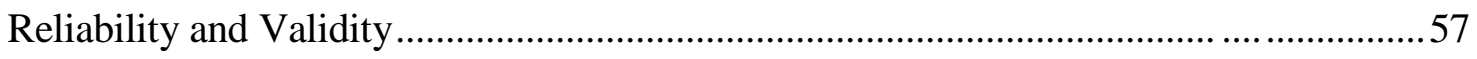

Rationale for Action Research...............................................................................5

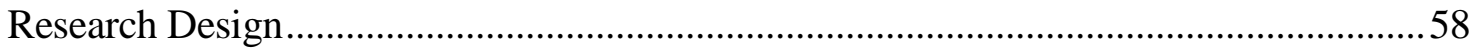




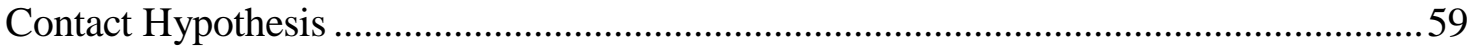

Research Preliminary Activities ............................................................................6

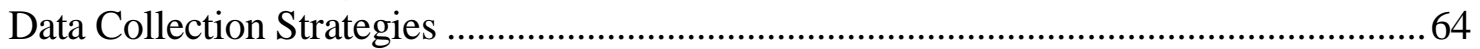

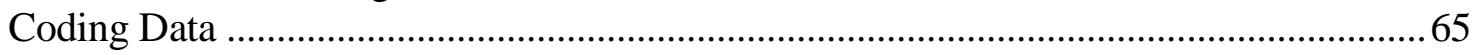

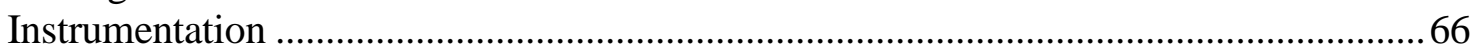

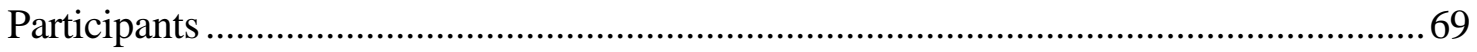

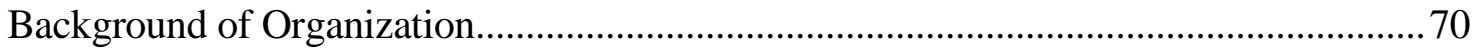

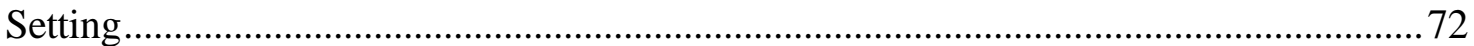

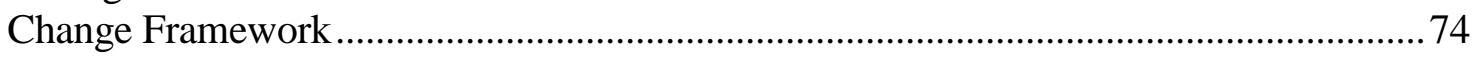

Researcher's Formulation of Personal Theory ...............................................................76

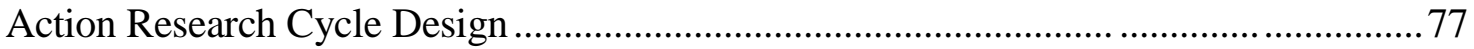

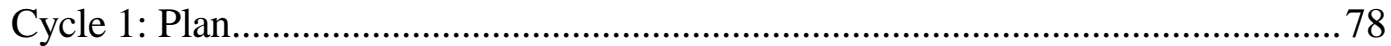

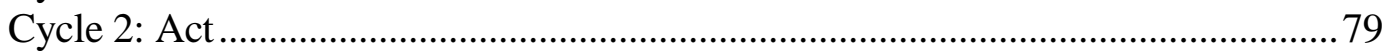

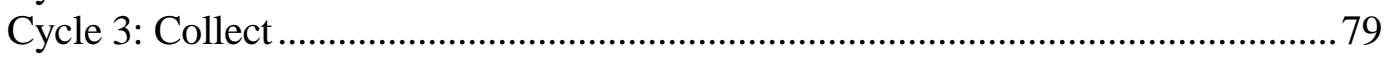

Cycle 4: Reflect, Interpretation, Informed Action ...................................................79

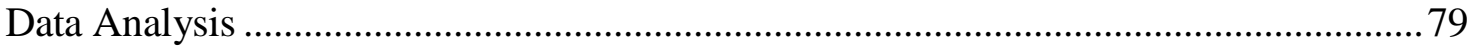

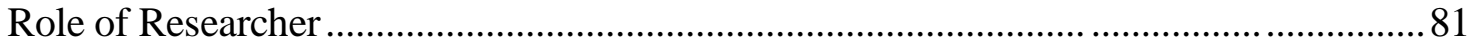

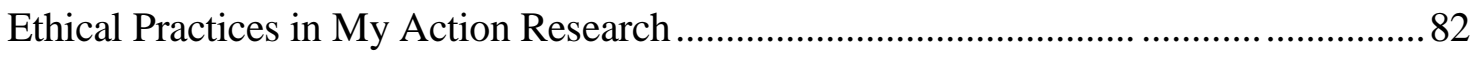

Discussion on Paradigm Design...................................................................................... 83

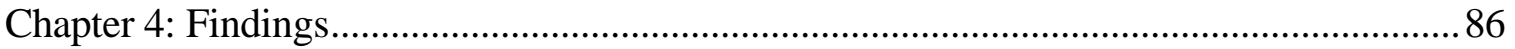

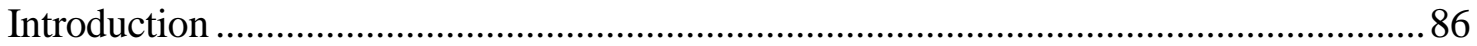

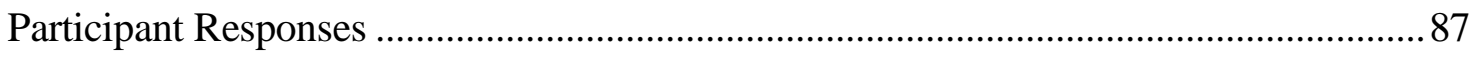

Survey Response: Subsidiary Analytical Research Question \#1. .....................................8 89

Learning Reflections \& Feedback: Subsidiary Analytical Research Question \#2 .........100

Interview Responses: Subsidiary Analytical Research Question \#3 ...............................110

Procedures for Answering Research Questions ............................................................111

Findings Supporting Primary Research Question .........................................................111

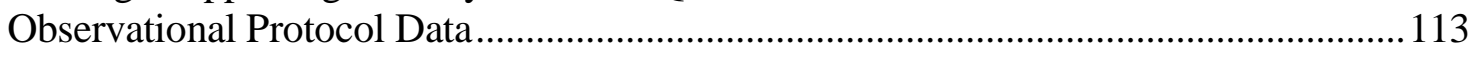

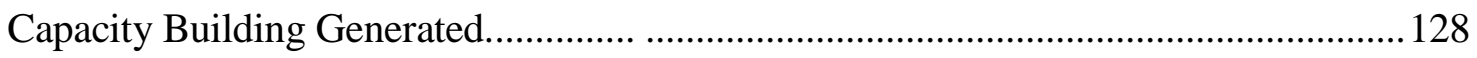

Six Month Follow Up: Reflective Practice ................................................................128

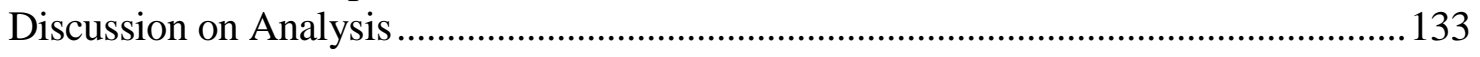

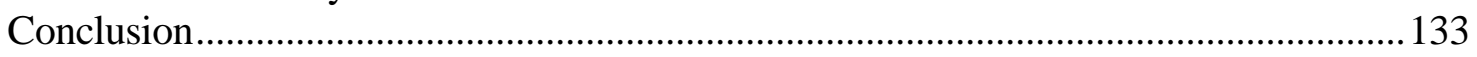

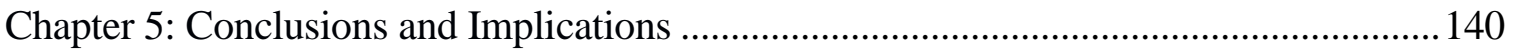

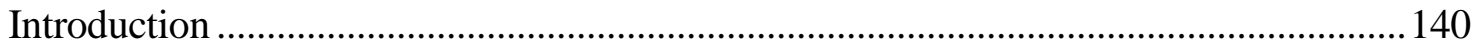

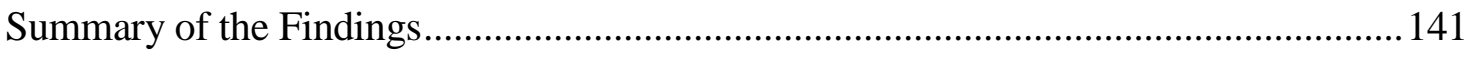

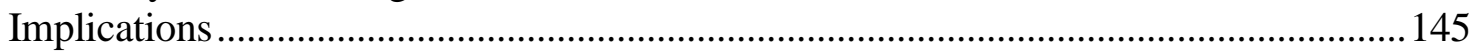

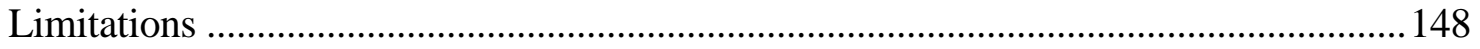

Recommendations for Informed Practice................................................................... 149

Directions for Future Research Study .......................................................................... 149

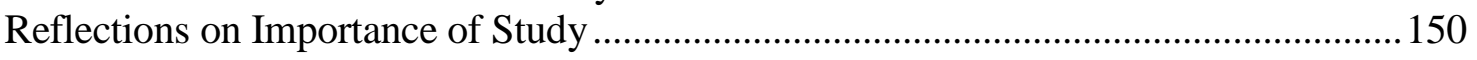

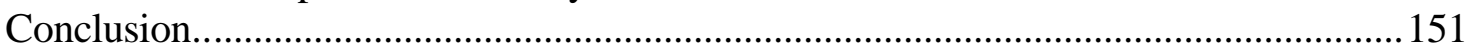

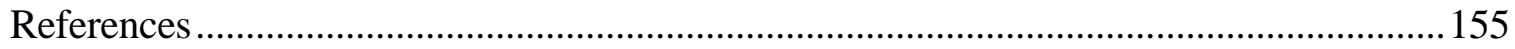


Appendix A: Letter Requesting Permission/Permission Granted...................................165

Appendix B: Consent Forms — Informed Consent \& Consent for Survey.......................167

Appendix C: IRB Application's Protocol Approval Letter...........................................170

Appendix D: Participant Pre Assessment Survey Responses Summary..........................172

Appendix E: Participant Post Assessment Survey Responses Summary.........................177

Appendix F: Participant Learning Reflections Survey .............................................182

Appendix G: Coding Document (central office administration interview protocol)........185

Appendix H: Coding Document (site administration interview protocol).......................188

Appendix I: Coding Document (participant observational protocol).............................192

Appendix J: Certificate of Completion................................................................... 197

Appendix K: Parallel Conversion Plan for Implementation Process................................199

Appendix L: Participant Six Month Follow Up Survey..............................................202 


\section{List of Tables}

Table

Page

2-1 Analysis of Variance Summary Table (Brown \& Carbonari, 1977) 35

2-2 Prejudice Reduction Workshop Model Framework 45

(Brown, 2009; NCBI, 2010 \& 2011)

4-1 Sample Coded Scheme: Interview (Central Administration Responses) 112

4-2 Sample Coded Scheme: Interview (Site Administration) 113

4-3 Sample Coded Scheme Workshop \#1: 3 Levels of Leadership 114

4-4 Sample Coded Scheme Workshop \#1: School Community as Peers 114

4-5 Sample Coded Scheme Workshop \#2: Environmental Scanning 115

4-6 Sample Coded Scheme Workshop \#2: Ups/Downs Activity 115

4-7 Sample Coded Scheme Workshop \#3: Stereotyping: $1^{\text {st }}$ Thoughts 117

4-8 Sample Coded Scheme Workshop \#3: First Thoughts Exercise 117

4-9 Sample Coded Scheme Workshop \#4: Internalized Oppression 119

4-10 Sample Coded Scheme Workshop \#4: Controversial Issues Process 119

4-11 Sample Coded Scheme Workshop \#5: Building Pride: "It's Great To Be" 121

4-12 Sample Coded Scheme Workshop \#5: Building Capacity 121

4-13 Sample Coded Scheme Workshop \#6: Becoming Allies 123

4-14 Sample Coded Scheme Workshop \#6: Caucus Groups 123

4-15 Sample Coded Scheme Workshop \#7: Making Commitments to Change 124

4-16 Sample Coded Scheme Workshop \#7: Speak Outs 125

4-17 Sample Coded Scheme Workshop \#8: Shifting Attitudes 126

4-18 Sample Coded Scheme Workshop \#8: Role Playing and Skits 126 


\section{List of Figures}

Figure Page

2-1 General Model for Prejudice Reduction Strategy, Selection, 18 Implementation and Evaluation (McKown, 2005)

2-2 Theoretical Framework Model. Basic cycle of experiential learning (Knowles \& Cole, 1996)

2-3 Theoretical Framework Model. Cyclical and spiral experiential learning 28 framework (Knowles \& Cole, 1996)

2-4 Conceptual Framework Map as adapted from Clark (2004) 37

3-1 Researcher's Proposed Change Theory Model 76

4-1 Bar Graph: Participant Pre Assessment Question $1 \quad 89$

4-2 Bar Graph: Participant Post Assessment Question $1 \quad 90$

4-3 Bar Graph: Participant Pre Assessment Question $3 \quad 91$

4-4 Bar Graph: Participant Post Assessment Question $3 \quad 92$

4-5 Bar Graph: Participant Pre Assessment Question 5

4-6 Bar Graph: Participant Post Assessment Question $5 \quad 94$

4-7 Bar Graph: Participant Pre Assessment Question $6 \quad 95$

4-8 Bar Graph: Participant Post Assessment Question $6 \quad 96$

4-9 Bar Graph: Participant Pre Assessment Question 9

4-10 Bar Graph: Participant Post Assessment Question $9 \quad 98$

4-11 Bar Graph: Six month follow up Survey Question 1 130

4-12 Bar Graph: Six month follow up Survey Question 2

4-13 Bar Graph: Six month follow up Survey Question 3 


\section{Chapter 1}

\section{Introduction}

\section{Research Problem Statement}

According to Mills (2011), action research is defined as "any systematic inquiry conducted by teacher researchers, principals, school counselors, or other stakeholders in the teaching/learning environment to gather information" (p.5). Action research is therefore an investigative means of inquiry involving informed practice. According to Gephart \& Marsick (2003), the action research approach is best in building capacity for learning and change; whereas, by using inquiry the researcher is able to find out more about the social system in which we function while attempting to resolve problems within them.

The makeup of the research problem looked at capacity building for learning and organizational change (Brown, 2009; Epstein, 2004; Gephart \& Marsick, 2003) in order to effectively learn about improving the school environment. Specifically, the conceptual framework provided insight on what factors or variables promote organizational change through effective informed practice (Willard-Holt, 2000). The researcher as participantobserver analyzed how prejudice behavior could be reduced from a Performance Improvement perspective (Clark, 2004) in the form of a diversity leadership skills training paradigm model adapted from Brown's model (2009) and continuum plan; henceforth, embedded in a school improvement plan for suggested implementation.

Historical roots of this controversial issue in regards to the prejudice reduction

process spans back over 40 years in the Civil Rights movement to include the 1972 Civil Rights Act, Title IX. This act prohibited racial discrimination against students and staff in 
public education. Likewise, this issue stems further almost 60 years when the public schools system was charged during the 1954 Supreme Court ruling in the case of Brown vs. Board of Education (Corwin, 1978 as referenced in Huff, 1998). Through the collaborative results of the Brown decision and Title $I X$, the actions taken were in hopes of reducing prejudice and discriminatory behaviors. The expectation was that the effects would afford regulatory policy, holding educational stakeholders, administrators and instructional leaders alike accountable for the evident and hidden racism practiced within the school environment.

School improvement planning is an intervention strategy used in addressing the social justice issue of prejudice reduction. The overall objective of school improvement planning is an enhanced level of student achievement through an enhanced leadership framework and successfully implemented program. School improvement plans are organized around three key areas: Curriculum Delivery, School Environment, and Parental Involvement. This study was organized around the school environment with focus on prejudice and discriminatory behavior reductions. Educators, educational administrators, and board members of educational services have internalized prejudice. Whereas, this study and its research project activities application were important by examining the presumptions within the school that create tension and conflict (Brown, 2009; NCBI, 2010 \& 2011). The improvement plan process requires focus on specific priorities to effect real change. The role of the principal or designee administrator is to collect material that will help the planning team determine a priority area of opportunity for growth in these key areas. 
Reed (1996) maintains that discrimination often occurs "when people believe themselves to be superior based on their race, gender, socio-economic status" (p.81) or some other private identity, hidden group. Reed asserts that the organizational framework of our schools in the United States as well as educational leaders' ideologies are able to bring about conceptualized precepts of change by using informed practice methods, educational policies, procedures and political frameworks (Bolman \& Deal, 2003; Reed, 1996). Reed argues that it is imperative for school leaders to strategically reduce the conscious and unconscious discussions that espouse presumptions on self importance.

The early educational agency where the study was conducted is a minority corporation whose members have all experienced the effects of prejudice; and many believe they are not prejudice. According to Purkey (1992), self concept theory is important to helping one to understand that behavior is influenced by the manner in which a person perceives himself or herself. Therefore, these perceptions function as both antecedent and consequence of human activity (Purkey, 1992). In that, the leadership skills application learned during the eight week training aimed to understand how such presumptions impacted participants' actions and attitudes. Moreover, skills application explains how we can change these presumptions within the learning environment as a person's beliefs drive their professional practice.

Prejudice reduction practices and their application for respecting diversity in educational leadership helps to create a safe and welcoming learning community for its entire member (Brown, 2009). Prejudice reduction education programs include multicultural curricula and discussion groups. The purpose of the prejudice reduction discussion groups are to learn how racism and other -isms affect people, develops an 
individual's own cultural and self awareness, evokes empathy for others, builds pride and capacity (Brown, 2009; Gephart \& Marsick, 2003; Waltzer, 1987 as referenced in Freeman, 2010). These are applicable skills needed to fight prejudice as well as address the internalization of negative stereotypes on all groups such as ethnicity and nationality of men and women, alcoholics, sexual orientation, as well as age, creed, race, and geographic backgrounds.

\section{Impetus for Study}

As an undergraduate student at Rutgers University, I completed one of the first comprehensive research papers on the history, purpose, and organizational leadership structure of the research site's parenting corporation. The foundations of that initial compilation of written research work became a contributing archived document for the agency. Consequently, the information from the original authored work has developed 20 years later into an expanded primary area of focus on prejudicial and discriminatory behavior and conduct. It is with this foundational work that I was able to use my skilled and reflective learning practices to transform this knowledge into a scholarly written dissertation on diversity in educational leadership. Other interest stemmed from my observed interactions amongst employees, children and families in addition to changes in the parenting agency's organizational framework and service population while I was transitioning into various administrative roles over a span of 20 years.

In addition, the agency's program has successfully served over 300 children and their families. Programming included comprehensive child care services for parents who are gainfully employed, in training programs, and who are seeking to further their education. This agency has assisted parents in this aspect by exceeding its initial 
objective. Whereas, founding program goals were originally to benefit the needs and concerns of a predominant racially identified population. Today, the population served is a diverse multicultural and multiracial community population of children and families. The founding agency's initial program was located in a community that is economically, linguistically, racially and ethnically diverse.

\section{Evaluation and Inquiry Approach as Intervention}

The researcher utilized pedagogical and participatory approach of inquiry through the initial assessment of participants attitudes and dispositions, using pre and post assessment instrumentation in addition to engaging participants "in identifying and naming injustices or forms of oppression present" (Freeman \& Vasconcelos, 2010, p.8). Brown (2009; NCBI, 2010 \& 2011) confers that this school application of evaluation and inquiry as exercised and modeled through training workshops in exploring ways to contradict and to interrupt prejudice behavior. Likewise, a qualitative analysis of social interaction was conducted by Henze (2001) who examined 21 case studies, where school leaders had taken action to improve social relations amongst the school's culturally diverse learning community. Henze found that conflict included segregation, racism, socialization, and inequality were the dilemmas encountered in the study. Differential treatment, based on race, was a staff issue of concern while the composition of ethnic clubs was a student concern (Henze, 2001). The case study's findings concluded that "school leaders need to decide that intergroup relations are a priority area in education, and take actions appropriate to their role" (p. 17). Henze summarizes the actions to be taken by school leaders to improve social relations. Priority consideration was to develop a plan of action on how the school will address conflict to include effective approaches designed to address 
controversial issues. Recommendations for the approach requires everyone knows what the vision is, requires that they have different structures that are known to all. Therefore, the learning requires that the individual is known and respected to ultimately promote a welcoming and safe school environment.

More and more cultural studies scholars are accepting prejudice reduction, or anti bias education, as a subject worthy of academic consideration and its findings of contributing research (Carpenter, Zárate, and Garza, 2007; McKown, 2005; Paluck and Green, 2009; Pang and Park, 2003; Singh, 1991; Spiotta, 2004; Titus, 1998). Scholarly discussions have been presented and empirical studies on multicultural education have been found useful in establishing commonalities of appreciative inquiry (Aguilar, Mantel, Maslowski, McDaniel \& Miller, 2004) into cultural and prejudice simulations (Banks \& Banks, 1995; Brown \& Carbonari, 1977; Butt \& Pahnos, 1995; Gimmestad \& De Chiara, 1982; Lynch, 1987; Pate, 1995; Short, 1993). According to McKown (2005), peer discussions regarding the process of assessment, intervention selection, implementation, and summative evaluation have been reviewed and found instrumental in evaluating ethical and pedagogical issues, attitude changes as well as behavior outcomes of education to include developmentally appropriate and best practices in teaching and learning and informed practice (Freeman \& Vasconcelos, 2010).

McKown (2005) refers to research originating in developmental and community psychology that explores the relationship between diverse frameworks and practices in the social environment as well as individual thought, feeling, and behavior. Similarly, critical social theorists (Freeman \& Vasconcelos, 2010) support the concept of evaluation and inquiry approach. This process consists of the following: (1) engaging stakeholders 
themselves in identifying and naming injustices or forms of oppression present; (2) assessing practices from a critical perspective involves learning new ways of perceiving people's roles and locations in the perpetuation and resistance of oppressive structures; (3) the development of new understanding is contingent on changes in practices and material conditions, and cannot rely on rhetoric alone (p.8).

Furthermore, Kupetz (2008) argues that the way in which one deals with and interpret what one observes as different are affected by a variety of influences. Kupetz explains further, indicating such influences may also encompass what they see and hear in their homes, at school, and in their neighborhoods; what they view in the media; and what they are told by friends, relatives, neighbors, and teachers. Kupetz further points out those instructional leaders who teach in the early education professions are instrumental in promoting diversity in the early educational classroom (Kupetz, 2008). Diversity in the classroom, according to Kupetz, is most successful when appropriately modeled and presented creatively in order that the students fully appreciate the beauty in the similarities and differences amongst themselves.

In considering this action research study was conducted in an early education setting, Wardle (2007; 1999; 2003a; 2003b) asserts that children need to feel a sense of security and familiarity with the people within their emotional environment, while also taking pleasure in discovering and exploring new experiences that are unknown and unfamiliar to the child. Lastly, Wardle (2003b) agrees with Kupetz (2008), in that, he believes one way to encourage an acceptance and the differences of others in children is to expose them to the new and diverse learning environment. Another method is for teachers 
and administrators to model enjoyment and peaked interest in discovery, exploration, and uncertainty at all levels of education.

\section{Purpose of Study}

The purpose of this study was to assess and analyze the methods used by individuals and groups (Freeman, 2010; Freeman \& Vasconcelos, 2010; McKown, 2005) to solve social justice issues involving diversity in educational leadership. The exploratory approach (Mills, 2011) was used to best investigate the research problem, and provide insight to the researcher. The researcher's exploratory paradigm usage entailed the collection of qualitative data (interviews, observational protocols, group discussions, reflective journaling, etc.) to analyze and identify themes as well as quantitative data collection (survey) to measure the impact of identified themes discovered in the research.

The researcher's endeavor was to observe how support of the Brown (2009) model was in alignment to understanding its impact in three areas: (a) the three levels of leadership; (b) school community as peers; (c) overall participation. These three areas had an ultimate impact on the success of leadership towards resolving the actions of diversity. Brown's (2009) Prejudice Reduction Workshop Model is a useful resource tool for establishing and implementing a multicultural curriculum. Whereby, this intervention assessment was carried out by measuring the impact of professional development peer training on adult faculty/staff and their dispositions toward sustained behavior change in practice over time. This information obtained by the researcher provided baseline data, which laid the groundwork to develop a school improvement plan.

Moreover, the researcher as participant observer took into consideration the salient factors impacting prejudice reduction practices and the researched controversial issues 
surrounding behavior and conduct in urban education. By utilizing a conceptual mapping approach as adapted by Clark (2004), the researcher was able to provide a visual performance typology in identifying what behaviors and actions impact prejudice reduction practices. These primary identified factors included: levels of leadership, the significance of environmental scanning, stereotyping, internalized oppression, building pride and capacity, becoming allies, making commitments to change, and shifting attitudes.

Another purpose of this study was to explain how prejudice reduction practices as they relate to leadership training skills and the framework for schools' application for respecting diversity are essential in creating a safe and welcoming professional learning community for all its members. In addition, the outcome hoped for was to look at the observed impact of the professional development training on adult faculty/staff and disposition toward sustained change in practice over time, not the impact of informed practice on children's training. The reactions and the degree of real change in the children's attitudes, beliefs, actions and or behavior were not intended to be measured in this study.

Contributions to existing literature in support to the proposed goals and objectives for prejudice reduction practices, particularly in urban education, are modeled after Haberman's (1994) five step approach to facing prejudices: (1) analyzing prejudices in order to recognize the misinformation that people have learned about various groups; (2) seeking the sources of the beliefs to identify and heal from internalized oppression while the discrimination members of an oppressed group target at themselves and each other; (3) examining the benefits of prejudice to celebrate similarities and differences as well to claim pride in group identity; (4) considering the effects of prejudices to understand the personal 
impact of discrimination through the telling of stories; (5) planning to eliminate prejudices to learn hands on tools for dealing effectively with bigoted comments and behavior.

Likewise, the researcher designed the study to assess and collect the evidence of attitude change using, pre and post training Likert type assessments provided to focus group. Surveying and observational protocol methods (Creswell, 2007) on study's participant members, using a minimal group paradigm to include peer trainings, professional development diversity workshops were used with interview protocol methods. For the central office school administrators and site supervisors, participants were interviewed as well as participated in the action research through the inclusion of a Board member. This information obtained by the researcher provided baseline data, which laid the groundwork to develop a school improvement plan. Additionally, the information obtained in this study would later be used as a resource tool used for developing the organization's short term smart goals (DuFour \& DuFour, 2010). An implemented professional development plan would include the researcher continuing to serve in the role of consultant.

The action research project helped in part the researcher as participant observer in assessing the role of educational leadership and training skills for effective diversity and inclusion (Brown, 2009; NCBI, 2010 \& 2011). The researcher as participant observer endeavored to formulate a personal theory with the intent to capture key components amenable to empirical testing. This formulated personal theory model is covered in Chapter 3.

Lastly, the researcher's intent was to analyze and examine the data of this research on discovered aspects of prejudice reduction practices and create a proposed theory model 
on prejudice reduction for leadership training in an educational setting; thus, identifying whether the variables discussed are actual contributing factors surrounding a successful program. The aim was to identify the contributing factors from collected data gathered from the assessments administered. The study's findings as presented were endeavored to create a descriptive action plan/methodology for school implementation as suggestive for future research based on conclusions discussed in Chapter 5.

\section{Research Question}

How does diversity leadership training skills on prejudice reduction result in changed beliefs of discriminatory behavior in schools? The researcher's intention was to explore this question, using a systematic inquiry means of survey, observational and interview protocol methods (Creswell, 2007), and pre/post assessments. Furthermore, the researcher's intention was to explore this research question as a participant observer and monitor successful implementation practices over an anticipated four to six month term to include coded data findings, evaluation and assessment, training through reinforced professional development sessions, leadership skills training modules, and observation

protocols. Analyze areas of opportunity for growth and development were presented for a proposed improvement plan for some future recommended post study continuum based on qualitative embedded data collection strategies used and discussed.

\section{Subsidiary or Analytic Questions}

\section{What are the effects of informed practice on organizational attitudes and} behavior outcomes of education? 
2. What factors promote changes in reduction practices of prejudice behavior in schools? What factors impede these changes in reduction practices of prejudice behavior in schools?

3. Based on the data collected from the findings, how can the diversity leadership training be strengthened for the organization?

According to Mills (2011), action research is defined as "any systematic inquiry conducted by teacher researchers, principals, school counselors, or other stakeholders in the teaching/learning environment to gather information" (p.5). Action research is therefore suggestive in this process of informed practice. According to Gephart \& Marsick (2003), the action research approach is best in building capacity for learning and change; whereas, in getting to know more about the social system in which we function while attempting to resolve problems within them. Hence, the researcher's goal of this action research design was to establish whether or not the findings addressed in answering these research questions to confirm the contact hypothesis implied on informed practice through peer training and professional development activities.

Furthermore, the researcher's hope was to propose implementation of a multicultural education curriculum as well as a professional development continuum, embedded in a proposed comprehensive school improvement plan. This study took on the understanding that prejudice is learned and "that prejudice and interpersonal function can be changed through education" (Spiotta, 2004, p.15). Lastly, theories of the controversial issues process concerning prejudice reduction actions taken as presented and referenced by the researcher sought to relate informal and formal methods of diversity skills training. 
Reed (1996) agrees with this method of building capacity (Brown, 2009; Epstein, 2004; Gephart \& Marsick, 2003; Waltzer, 1987 as referenced in Freeman, 2010) within the school environment in helping to develop awareness of one another's untapped potentials (Reed, 1996, p.81).

\section{Significance of Study}

The action research approach (Gephart \& Marsick, 2003; Mills, 2011) attempted to address the measured impact of professional development training and whether or not effective skills application result in evidence of change (Kotter, 1996) over time in school leadership (Fullan, 2001), attitudes, and behavior outcomes (Denzin \& Lincoln, 2005) of informed practice in education (Freeman \& Vasconcelos, 2010). The significance of study and its implications will be of value to the organization's levels of leadership in considering self awareness, others awareness in being an ally, and the school's awareness for taking action. By doing so these actions taken help with building capacity to create major change (Epstein, 2004; Gephart \& Marsick, 2003; Waltzer, 1987 as referenced in Freeman, 2010).

The study suggested that School Administration leaders of successful prejudice reduction programs have established a curriculum of responsiveness to the learning community's needs, like multicultural education and enhanced leadership, through caucusing for groups within the urban school environment (Brown, 2009; Willard-Holt, 2000). Lastly, the diversity leadership training skills through practiced prejudice reduction and their application for respecting diversity became essential for building a caring school environment and promoted positive self image. The study in part enhanced the quality of the education programming for optimal student learning as well as 
encouraged community awareness and involvement. Lastly, the study adds to the research literature in professional development training of prejudice reduction among school personnel.

\section{Organization of Study}

This study is organized around a descriptive report format, using an action research design and qualitative embedded data collection strategies. Chapter One introduces the background information that helped define the research problem and why the study was conducted. The purpose of the study and its intended significance are explained as well as research questions posed. Chapter Two presents the Literature Review and theory that support diversity leadership skills training for schools, conceptual and theoretical approaches, and strategic interventional prejudice reduction practices taken. Literature relevant to this study is also reviewed in Chapter Two. The methodological approach, the group paradigm, and instrumentation used in data collection strategies are in Chapter Three. Chapter Four presents the researcher's analysis and report on the collected, coded data, its findings, and interpretation of the findings. Finally, the researcher's summary of findings, implications, recommendations for a proposed plan of action, and recommendations for further research study on informed practice is presented in Chapter Five. 


\section{Chapter 2}

\section{Literature Review}

The literature review will attempt to synthesize existing selected research in prejudice reduction, specifically in urban education. It will also intend to discuss shared findings of previous studies in anti-bias educational practices, areas of scholarly disagreement, and suggestions for future research. Additionally, theories of the controversial issues process concerning prejudice reduction actions taken will be referenced as they relate to informal and formal methods of diversity skills training in educational leadership. The purpose is to capture its key components and point out relevancy as amenable to empirical testing.

Furthermore, the main aspects of the literature review will introduce and highlight key theoretical approaches used that facilitated the research project. In addition, historical and present literature on the relevancy of theoretical (McKown, 2005) and conceptual framework approaches (Brown, 2009; Clark, 2004) used are presented to help define the research problem. Likewise, literature on the physiology of the research problem, discussing capacity building for learning and organizational change (Brown, 2009; Epstein, 2004; Gephart \& Marsick, 2003), is covered in this chapter to address strategies geared toward intervention for the school improvement process as well as reframing organizational change (Bolman \& Deal, 2003).

Chapter 1 introduced the study, indicating how prejudice reduction education programs include multicultural curricula and discussion groups. Additionally, the chapter discussed purpose and significance of the study to learn how racism and other-isms affect people and assess the impact of diversity leadership skills training. Moreover, the 
understanding and current trend in multicultural education combines prejudice reduction practices and effective diversity training for schools. Therefore, this critical pedagogy approach (Freeman \& Vasconcelos, 2010) teaches a set of skills that are essential for effective conflict resolution (Carpenter, Zárate \& Garza, 2007; Klein, 1992; Lynch, 1987; McKown, 2005; National Coalition Building Institute, 2009; Pang \& Park, 2003; Pate, 1995; Spiotta, 2004; Titus, 1998) in addition to establishing a "process of assessing practices from a critical perspective involves learning new ways of perceiving people's roles and locations in the perpetuation and resistance of oppressive structures" (Freeman \& Vasconcelos, 2010, p.8). Additionally, the significance of developing pedagogical approaches maintains to "help participants unlearn harmful conceptions of self and others, while actively participating in constructing new forms of knowledge, including those of [social] justice" (Freeman, 2010, p. 2). Spiotta (2004) explains that the objective of the diversity training is to develop a continuum of activities with "the necessary enabling infrastructures that assist in the transference of knowledge gleaned from the training to concrete activity and practice" (p.15). Hence, cultural studies scholars as well as critical theorists to a greater extent are accepting prejudice reduction in urban education as a subject worthy of academic consideration.

\section{Theoretical Framework: Ecological Assessment}

In reviewing selected literature on multicultural education (Carpenter, Zárate \& Garza, 2007; McKown, 2005; Spiotta, 2004; Pang \& Park, 2003), the empirical research provides insight from a theoretical perspective (McKown, 2005). What the research suggests or emphasizes is that prejudice reduction has some relevancy in regards to selffocus and the relationship between varied structures and processes in the social 
environment and individual thought, feeling, and behavior of groups (Carpenter, Zárate \& Garza, 2007). In addition, Carpenter et al. (2007) and Sherman (2003) support the notion that highlighting differences while simultaneously focusing on the self reduces prejudice, although similarity mediates the relationship with those who are perceived as more different. Sherman (2003) further asserts that the researcher as critical theorist seeks to mediate the ideals of philosophy by notions of "freedom," "equality," "justice," and "reconciliation" [and] mediate society's prevailing practices and underlying tendencies" (p.188). Furthermore, the researcher as critical theorist seeks to "develop pedagogical approaches that help participants unlearn harmful conceptions of self and others, while actively participating in constructing new forms of knowledge, including those of [social] justice" (Freeman, 2010, p. 2).

Additionally, McKown (2005) approached his study by examining the application of ecological theory to advance the science and practice of school-based prejudice reduction interventions. Similarly, this suggested framework was used in support to the methodological approach for discussion in Chapter 3. The author acknowledges, "Several school-based racial prejudice-reduction interventions have demonstrated some benefit" (p. 1). McKown (2005) further maintains that ecological theory serves as a framework in which to understand the limits and to enhance the efficacy of prejudice reduction interventions. McKown (2005) found that using ecological theory, the three prejudice reduction approaches according to Pate (1995): social cognitive training, cooperative learning, and liberation psychology were examined in terms of their conceptualization to suggest specific strategies and enhance prejudice reduction efforts in the schools. McKown (2005) concluded that each of the intervention approaches discussed "fits a limited range of 
ecologies. Intervention combinations should be selected based on an ecological assessment, and ongoing monitoring and evaluation of program effects should inform modifications to the intervention design overtime” (2005, p. 186). According to McKown’s (2005)

approach, Figure 2-1 presents the suggested model strategy approach used for this study to enhance prejudice reduction practices (p. 186):

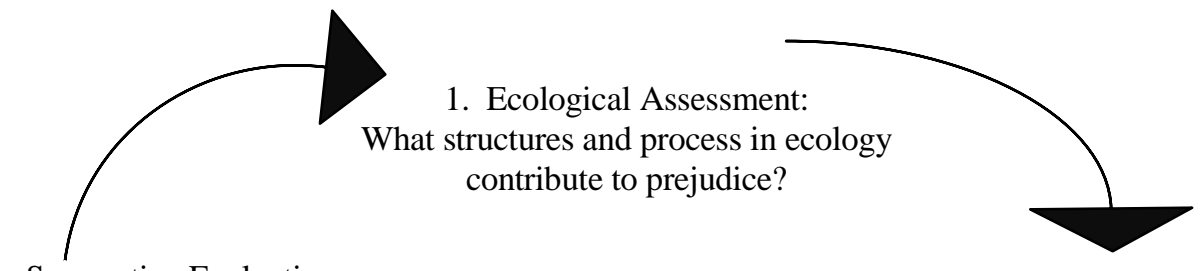

4. Summative Evaluation:

Did the intervention have the desired

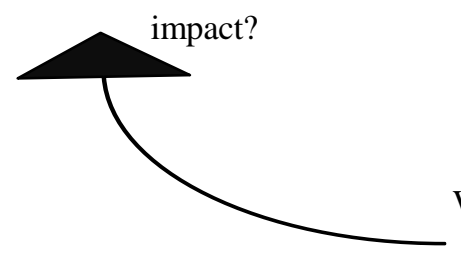

3. Implementation: What key players, resources, and strategies are needed to ensure intervention fidelity?

\section{What interventions fit needs identified in ecological assessment?}

\section{Figure 2-1 General Model for Prejudice Reduction Strategy, Selection, Implementation and Evaluation}

(McKown, 2005)

Overall, these empirical discussions on prejudice reduction strategies evaluate

ethical and pedagogical issues, attitude changes as well as behavior outcomes of education, including developmentally appropriate and best practices (Wardle, 1999; 2003a; 2003b). Prejudice reduction is a positive process whereby individuals resolve issues in an informal or formal atmosphere, or where issues are resolved as part of the ongoing interaction between individuals. Additionally, historical roots of this controversial issue in regards to prejudice reduction process spans back over 40 years in the Civil Rights movement to include the 1972 Civil Rights Act, Title IX. This act prohibited racial discrimination against 
students and staff in public education. Likewise, this issue stems further almost 60 years when the public schools system was charged with discovery of this research problem during the 1954 Supreme Court ruling in the case of Brown vs. the Board of Education (Corwin, 1978 as referenced in Huff, 1998). Through the collaborative results of the Brown decision and Title IX, the actions taken were in hopes of reducing prejudice behaviors. The expectation was that the effects would afford regulatory policy, holding educational stakeholders, administrators and instructional leaders alike accountable for the evident and hidden racism practiced within the school environment.

In considering McKown's (2005) strategic model approach and in order to accomplish my plan of action for the study, I modeled the theoretic framework of Kotter (1996) to address this awareness through his eight stage process. He explains how his theory-in-use for successful school improvement planning and implementation will assist in promoting real change. To effectively address the research problem, the overall objective of reducing prejudice behavior and conduct is to use the following eight stage process (Kotter, 1996):

\section{Kotter's Theoretical Approach (1996, p. 21): Eight Stage Process}

\section{Establishing a sense of urgency.}

\section{a. Examining the market and competitive realities.}

\section{b. Identifying and discussing crises, potential crises, or major opportunities.}

The salient factors or areas of critical concern impacting prejudice behavior and controversial issues in the school environment include: levels of leadership, the significance of environmental scanning, stereotyping, internalized oppression, building pride and capacity, becoming allies, making commitments to change, and shifting attitudes 
(Brown, 2009; Gephart \& Marsick, 2003; Haberman, 1994). The goal of a proposed school improvement plan change initiative would be to increase positive student behavior as well as awareness amongst the professional learning communities. Focus would be on prejudice behavior, including the frequency and type of inappropriate behavior (i.e., bullying, code of behavior; prejudicial jokes, comments, slurs, and other internalized oppressive behaviors) that impacts our own and different groups' self image (Purkey, 1992) and behavior.

\section{Creating the Guiding Coalition.}

\section{a. Putting together a group with enough power to lead the change. \\ b. Getting the group to work like a team.}

Through creating a powerful guiding coalition, parents, "student, faculty, and staff leaders will have the opportunity to come together to become more effective leaders and allies at your school" (Brown \& NCBI, 2009). This suggested change process "brings together people from all parts of the school community as peers" (Brown \& NCBI, 2009). According to Putnam, Gunnings-Moton \& Sharp, C. (2009), this group work setting also exercises excellent opportunity to practice effective group discussion skills.

Additionally, emotionally intelligent leaders must hold fast to their influences or passions expressed as empathy, understanding, caring, nurturance, and support. Therefore, a truly effective leader must balance "emotionally as well as intellectually" (Goleman, 2002, p. 208) his or her influence to achieve a shared vision in developing emotionally intelligent and high functioning teams. This is done through resonance with concern on the part of all employees for the sake and in favor of everyone's well being. What is most important is that the leader requires listening carefully and learning as people express what they want. In that action within itself, the leader needs to reinforce what is shared and what 
their different visions have in common. "It requires a direct connection with people's emotional centers" (Goleman, 2002, p. 208).

\section{Developing a (Shared) Vision and Strategy.}

\section{a. Creating a vision to help direct the change effort.}

\section{b. Developing strategies for achieving that vision.}

Through the application of Appreciative Inquiry (AI) approach (Aguilar, Mantel, Maslowski, McDaniel \& Miller, 2004), Emotional Intelligence-EQ (Goleman, 2002) and Reflective Practice (Schön, 1974) in supervision, are in essence a leader's "guiding and motivating [force] with a compelling vision", and these presented theories in particular are foundational in building "relationship[s] for learning" (Goleman, 2002, p. 39; Aguilar et al., 2004, p. 114). Sustaining commitment while maintaining a harmonious and cohesive (emotional) work environment keeps focus on the vision's common good. This sustained commitment is shared by followers for a solid organizational structure and furthermore inspires positive results.

Hence, "[a]n organization has desired outcomes reflected in its vision, mission and [core] values" (Aguilar, et al., 2004, p. 40). Moreover, this vision or "a mental image of a possible and desirable future state of the organization" (Bennis \& Nanus, 2003, p.82) is symbolically paramount for the attainment of a group structure's desired purpose. Lastly, building emotionally intelligent organizations involves collective input into the vision. Goleman maintains that " $[\mathrm{t}] \mathrm{o}$ create the vision...emotionally intelligent leaders need to cocreate the vision that will serve to rally and [inspire] the group as a whole" (Goleman, 2002, p. 206). 


\section{Communicating the Change Vision and Plan.}

a. Using every vehicle possible to constantly communicate the new vision and strategies.

\section{b. Having the guiding coalition role model the behavior expected of employees.}

To reiterate, everyone on the planning team should become familiar with the new vision and strategies for the school and its community. Revisions upon implementation of the training plan on the school profile will communicate the new vision and strategies within the District. Also, reviewing the school profile and discussing each area helps everyone involved in the planning process understand the school, and it sets a context of focused priority for the improvement plan. Finally, communication within the learning communities to promote modeled behavior (Willard-Holt, 2000) is expected of the leadership framework to provide practical skills that can be used in everyday situations to create a safer and welcoming school environment for all learners.

\section{Empowering Broad Based Action (Empower Others to Act on the Vision).}

\section{a. Getting rid of obstacles.}

\section{b. Changing systems or structures that undermine the change vision.}

\section{c. Encouraging risk taking and nontraditional ideas, activities and actions.}

Leadership is a process and not a position of leverage in getting rid of obstacles. Kotter (1996) asserts this statement in his argument; whereby, empowering broad based action is the grassroots approach to change management that creates ownership for the school improvement plan. This ownership is generated within the learning communities and reduces the barriers to that change. Encouraging the expression of pride in all groups and understanding the differences between authentic pride and prejudice will assist in 
taking action to build capacity as well as creating opportunities for parents, students, staff, and all professional learning communities to embrace positive change initiatives within the school environment.

Kotter (1996) further explains that we can break down those barriers and create a culture that encourages participation and risk taking through rewards, knowledge sharing, providing employees with opportunities to impact processes and the meeting of agency goals and promoting the successes of those contributions through training and recognition. Kotter \& Cohen (2002) contribute in their argument that "[i]n highly successful change efforts, when people begin to understand and act on a change vision, it is important to remove barriers in their paths, to take away the tattered sails and give them better ones" ( $\mathrm{p}$. 73).

\section{Generating Short term Wins.}

\section{a. Planning for visible improvements in performance, or "wins."}

\section{b. Creating those wins.}

\section{c. Visibly recognizing and rewarding people who made the wins possible.}

The authors (Fullen, 2001; Kotter, 1996; Kotter \& Cohen, 2002; Muhammad, 2009;

O'Brien, 2001) contend, real change does take time and it is important that all team members recognize this notion as they enter into the school improvement planning process. Incremental improvements are significant, and they should be celebrated, but they do not constitute lasting change. Kotter (1996) \& Muhammad (2009) agree that recognition is important when school leaders work with teams in building momentum for student focused school change. As a result, the rewards mechanism process requires changing to ensure that knowledge sharing is adopted by employees for the good of the organization (Kotter, 
1996). Professional development opportunities, Student/Faculty of the Month, and Teacher of the Year are such rewards mechanisms.

\section{Consolidating Gains and Producing More Change.}

a. Using increased credibility to change all systems, structures, and policies that don't fit together and don't fit the transformation vision.

\section{b. Hiring, promoting and developing people who can implement the change in vision.}

\section{c. Reinvigorating the process with new projects, themes, and change agents.}

Preliminary steps would be taken for consolidating gains and producing more change, leaving credibility charged to the represented learning communities instructional leaders and faculty in support of developing caucuses for allied group work (Wren, 1995, p. 327) determine a shared time for participant speak outs in efforts for making commitments to change (Brown, 2009), and work on role playing and skits with participants for effective shifting attitudes (Bolman \& Deal, 2003). Additionally, qualitative methodologies practiced by the researcher were used through observational and interview protocols (Creswell, 2007). The researcher used this method of inquiry to gather valid data and information (Auerbach \& Silverstein, 2003; Creswell, 2007; Rubin \& Rubin, 2005) for the study. This practice will not distract subjects since observation of pedagogy is periodic procedures conducted for quality management purposes as per educational policy administrative codes. Furthermore, suggested recommendation given are to collect observations made as kept in a journal report profile along with any previous observatory protocols (Creswell, 2007), evaluation reports and or surveys to use as final concluding 
analysis in evaluating and assessing newly implemented change initiatives and or skills training continuum applications (Stringer, 2007).

In addition, parent involvement and participation play a key role in consolidating gains and producing more change. Parents are an integral part of their child's education, at home and at school; as well school leaders recognize parents as a positive, welcoming, and necessary factor for a successful education environment. Without parent involvement, the chance of a program success is least likely to be achieved due to its contributing factor of the research problem. Participation is imperative in order to provide time for teaching staff to share information about the progress of children and to collectively work together in community support efforts for the agency's education programs as well as solving problem situations that may arise. Likewise the school's student, faculty, and staff support serves in an equal role along with parent involvement. Family and community partnerships are an information resource and a shared opportunity for obtaining assessed data feedback and input during the school improvement planning process.

\section{Anchoring New Approaches in the Culture.}

a. Creating better performance through customer and productivity oriented behavior, more and better leadership, and more effective management.

\section{b. Articulating the connections between new behaviors and organizational} success.

\section{c. Developing means to ensure leadership development and succession.}

In this final stage of the change process, additional suggested recommendations for creating better performance and monitoring system delivery (implementation and assessment) for tracking newly implemented plan may include the use of statistical 
software (i.e. SPSS, Statistical Package for the Social Sciences). This delivery system would be used to compile and review any significant output data for quantitative analysis purposes, if needed for future analyzed measureable indicators. Relevant demographic information on indicators of learners' well being in the State based on performance can be provided also for statistical purposes. Additionally, the researcher's methodology used to measure accountability was qualitative based, which included both formative and summative assessment as well as the following participant evaluation methods:

- observation protocol documentation;

- pre/post training surveys (attitudes and dispositions);

- personal reflection papers;

- interview protocols of stakeholders;

- quotes from personal reflections

\section{Theoretical Framework Model for Action Research}

Kotter's eight stage process (1996, p.21) encourages shared learning initiatives through professional development in alignment to organizational change (Muhammad, 2009). For example, Figure 2-2 and Figure 2-3 are adapted models used to illustrate this suggested theoretical framework as referenced as well by Creswell (2003). He explains the adapted framework used by the researcher as "theoretical lens[es]...to guide [our examination of] what issues are important to examine [and] how the final accounts need to be written" (Creswell, 2003, p. 131): 


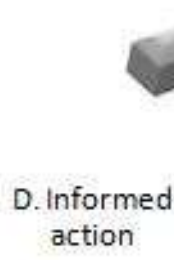

$$
\begin{aligned}
& \text { C. Reflection } \\
& \text { and analysis, } \\
& \text { formulation of } \\
& \text { personal } \\
& \text { theories }
\end{aligned}
$$
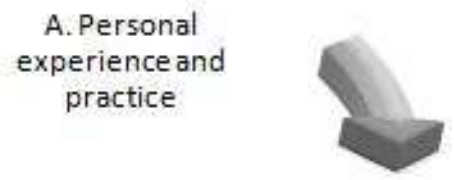

B. Information gathering and documentation

Figure 2-2 Theoretical Framework Model Basic cycle of experiential learning (Knowles \& Cole, 1996)

As illustrated, Knowles \& Cole (1996) provide the first part of the framework as shown in a basic cycle of experiential learning with four phases. Figure 2-2 shows the basic cycle, starting with the personal experience and practice of the learner. This is developed by information gathering and documentation (i.e. journals, observation and interview protocols, surveys, etc.) that assist the researcher in generating significant learning reflections and data analysis on the experience that will help them to formulate personal theories of teaching and learning as well as informed practice (Wells, 1994). These personal theories then help to inform on future practices of study in action research. As a result, the reflective learning experience is thusly enhanced (Figure 2-3): 


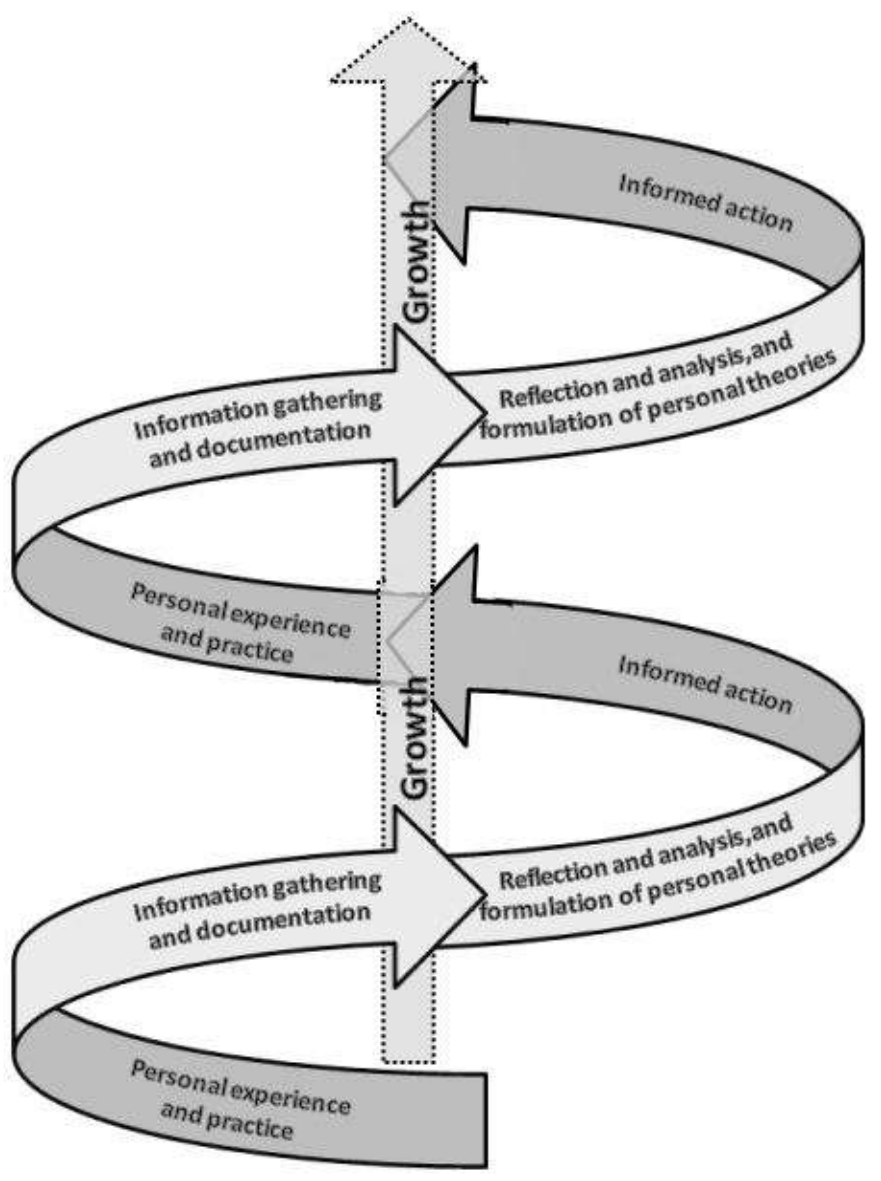

Figure 2-3 Theoretical Framework Model Cyclical and Spiral Experiential Learning Framework (Knowles \& Cole, 1996).

As shown in Figure 2-3, Compton \& Davis (2010) explain that the experiential learning framework also requires practitioners of action research to reflect and analyze their field experiences. Further, this framework suggests that practitioners compare their personal histories with new information gathered during the field experiences. The authors conclude, indicating that based on these reflections and analyses, personal theories (Figure 2-3), the researcher formulated a proposed change theory model conducive to teaching and learning as this relates to this research project with the intention of influencing future practices. 


\section{Overview of Action Research Cycles}

Moreover, action research is a form of qualitative research that explores problems in organizations by planning, implementing and reflecting on a particular process or educational phenomenon (Craig, 2008; McMillan, 2004; Mills, 2011; Stringer, 2007; Watts, 1985). Action research is participatory in design and is done by the practitioners for themselves where shared learning and ownership is practiced (Craig, 2008; Mills, 2011; Watts, 1985). Additionally, action research is cyclical, allowing the researcher the ability to change, adapt, and restructure in order to effect a fundamental organizational system change (McTaggart, 1997; 2000). Lastly, Compton \& Davis (2010) explain that as the researcher completes each cycle, the reflective learning experience increases in complexity, and this helps to promote the growth of the learner (Knowles \& Cole, 1996).

\section{Action Research \& Professional Development}

According to Brown (2009; NCBI, 2010 \& 2011), student, faculty, and staff leaders now have the opportunity through action research (Craig, 2008; Gephart \& Marsick, 2003; Stringer, 2007) and professional development training (DuFour, DuFour, Eaker \& Many, 2006; Kotter, 1996) to work together to become more effective leaders and allies while practicing the skills learned at their school. This professional development leadership program is unique in many ways. Brown, Mazza \& NCBI (1998; 2005) support the concept of leadership for diversity through action research study (Craig, 2008; Gephart \& Marsick, 2003; Mills, 2011; Stringer, 2007). Diversity leadership training brings together people from all parts of the school community as peers and provides practical skills that can be used in everyday situations while effectively building capacity to create as well as sustain a 
safer and welcoming school environment for all (Brown, 2009; Brown \& Carbonari, 1977; Henze, 2001; Klein, 1992; Lynch, 1987; Paluck \& Green, 2009).

\section{Critical Social Theory}

According to Freeman \& Vasconcelos (2010), critical social theory is significant in particular to supporting the evaluation and inquiry approach in action research. The theorists maintain that engaging the stakeholders themselves in identifying and naming injustices or forms of oppression present is essential in the papticipatory approach. In addition, the pedagogical process of assessing practices from a critical perspective involves learning new ways of perceiving people's roles and locations in the perpetuation and resistance of oppressive structures (p.8). Furthmore, the evaluation and inquiry approach is significant to critical social theory for being action oriented. Whereby, the development of new understanding is contigent on changes in practice and material conditions, and cannot rely on rhetoric alone (Freeman \& Vasconcelos, 2010, p.8). Consequently, the researcher as a critical theorist seeks to "develop pedagogical approaches that help participants unlearn harmful conceptions of self and others, while actively participating in constructing new forms of knowledge, including those of [social] justice" (Freeman, 2010, p. 2). Finally, the researcher as social scientist is connected to and cares deeply about the issue [she] seeks to change (Waltzer, 1987 as referenced in Freeman, 2010, p.3).

\section{Capacity Building}

According to DuFour, DuFour, Eaker \& Many (2006) and Kotter (1996), the authors maintain that learning by doing and short term gains are effective capacity building strategies. The capacity building intervention measures are best practiced through developed collaboration, strategic planning, coalition building, and sharing information 
(Hambright \& Diamantes, 2004; Kotter, 1996). Other capacity-building strategies from an organization development standpoint emphasizes and supports Kotter's (1996) change framework approach through incorporating intervention practices of formative and summative assessment of services and needs, training of professionals and or stakeholders, increased access to outside resources, and technical assistance in using data to improve services. Consequently, the researcher was successful with engaging participants in shared learning opportunities through the professional development activities and issued contact hours as incentive for their time. Kotter states that "Short term gains are needed to establish credibility for a change initiative over the long haul. Major change takes times. Most of us want to see some convincing evidence that all the effort is paying off. We want clear data, indicating changes are working" (p. 118-119).

In addition, capacity building according to Epstein (2004) involves stakeholders' systems thinking at all levels: at Board level, learning to solve problems and manage dilemmas; at the Superintendent level, being better political, managerial, school administrators; at school level, practicing principals who serve in the capacity of instructional leaders and curriculum supervisors; and lastly, at classroom level, as professional educators and teaching experts in knowledge and skills (p.122). Overall, the strategy for capacity building should include short-term objectives for early success and support (DuFour et al., 2006; Kotter, 1996). The next section will provide some insight into the sociology of the research problem involving cognitive interactions of stakeholders.

\section{Sociology of Problem: Models of Cognitive Interaction}

Woodard (1966) discusses his research on the theoretical viewpoint principle of belief congruence and the congruity principle as models of cognitive interaction in 
alignment to prejudice reduction practices. According to Rokeach \& Rothman (1965), belief congruence is based upon a 'theory' of prejudice, which suggests that the principle determinant of an individual's attitude toward another individual is the similarity or congruence between the two individuals' belief systems. Woodard suggests based on Rokeach \& Rothman's theory (1965) that where there is great similarity, mutual attraction is thought to ensue; dissimilarity is presumed to lead to rejection. His study had combined social-psychological theory and empirical methodology. Woodard's tested hypothesis implied "that Negro and White parents of similar socio-economic status would not differ significantly in their attitudes toward junior high schools of varying racial balance" (p.1). The study was done in Buffalo, New York. Woodard generated this theory based upon Rokeach \& Rothman's (1965) formulation that belief congruence surpasses racial disparities. Although the theorists present opposing viewpoints, the implications of each suggest that further evaluation and inquiry is needed for research.

However, the hypothesis failed to prove this assertion; whereas the empirical data received from Woodard's individual surveys of 80 Negro and 70 White randomly selected parents did not support the hypothesis (Woodard, 1966, p. 169). Woodard's comparative analysis of the mean scores concluded "that Negros and parents with a great deal of education had lower mean acceptance scores than Whites and parents with less education" (p.169). He found that both race and education impacted attitude of the parent scores.

Consequently, Woodard argued that Rokeach's original study and suggested theory "did not sufficiently concern itself with concrete, real life issues - at least not to the extent the present research did. That is, the theory does not seem to hold for this kind of school integration issue" (p.173). Woodard further concluded from his direct surveys that his 
concrete questions in this study may have been ascribed as a primary "underlying threat to self-esteem and/or status" (p. 171). Hence, this implication extends the notion to suggest the early beginnings of prejudice reduction actions taken to enhance an emerging leadership framework enhance an emerging leadership framework used to ameliorate prejudice, handle controversial issues and perceptions surrounding diversity in educational leadership.

Similarly, another study completed a decade later was based on a multicultural project designed to reduce group isolation. This study provided more positive results in attitude and behavior outcomes, specifically among school-age children. Attitude changes toward the cultural diversity and ethnic differences were measured through the use of doll play. The study group involved a selected sample of school-age children from first through sixth grade where school demography entailed a significant sample of Black, Anglo, or Mexican American children (Brown \& Carbonari, 1977), all who resided in Houston, Texas. The study was conducted at the People Place of Houston, a multicultural center that provides "racially isolated elementary school children with exposure to various world cultures" (p.1). A random sample of children from the groups coming to People Place consisted of three student ethnic groups: a Black child, an Anglo child, and a MexicanAmerican child. These children were selected from the second and fourth grade. Again, the behavior outcomes favored positive attitude changes with the children who participated in the People Place study in comparison to the control group observed. The study total sample consisted of 66 children equally divided among these three ethnic groups. However, the literature did not specify who was excluded from the study activities. 
Brown \& Carbonari (1977) further explain that the "control group proportional with respect to ethnicity and grade level attending a school function of similar length to that of People Place experience, but not involving any cross cultural or multi-cultural experience, were given the same situational doll play evaluation instrument" (p.5). Also explained was that "measures were also taken on them before and after their experience of this school function" (p.5). The control group consisted of 30 children, totaling 96 children for the sample. Notably observed was that Black children exhibited significantly more attitude change than Mexican Americans; however, the extent of change with the Anglo children "was not significantly different from either of these groups" (p.8). The authors asserted that the white children were able to differentiate their racial identity among the three dolls.

The setting of the study was situated in a simulated classroom, developed so that each child could express his or her attitude by arranging these culturally diverse dolls (Black, Anglo, and Mexican American) in a play-like environment. The dolls were six inches in height, representing each child's ethnicity. The main discriminator was the actual physical color of the doll. Each child was given individual instruction to position the three dolls in the simulated room in order and then wait for the teacher's return. Indicator marks were written on paper on the simulated play room's floor to denote the position of the three dolls as placed on the paper by each child. Also denoted on the paper was the race and grade level of each child.

When a new child entered the simulated environment, a new sheet of paper was placed on the simulated play room's floor as the scoring sheet for the next child. The indicator markings "became the raw proximity data to later be used in the analysis as a pretest measure" (p.4). The data cited in Table 2-1 reveal a quantitative summary analysis of 
variance results. As shown, the mentioned "significant differences were found as a function of the treatment and as a function of the ethnicity of the child" (p.7):

Table 2-1

Analysis of Variance Summary Table (Brown \& Carbonari, 1977).

\begin{tabular}{lcccc}
\hline Source & SS & DF & MS & F \\
\hline Ethnicity & 712.335 & 2 & 356.168 & $3.225^{*}$ \\
Grade & .766 & 1 & .766 & .007 \\
Group & 1736.40 & 1 & 1736.40 & $15.723^{*}$ \\
Ethnicity by & 416.09 & 2 & 208.04 & 1.82 \\
Grade & & & & \\
Ethnicity by & 583.69 & 2 & 291.84 & 2.56 \\
Group & & & & \\
Grade by Group & 6.50 & 1 & 6.50 & .05 \\
Ethnicity by & 185.80 & 2 & 92.89 & .81 \\
Grade by Group & & & & \\
Within & 9553.422 & 84 & 113.73 & \\
$* \mathrm{P}<.05$ & & & & \\
\hline
\end{tabular}

The quantitative data shown in Table 2-1 as interpreted by Brown \& Carbonari (1977) revealed that the children's experience in this study did change attitudes of the selected sample of children at the People Place of Houston. The use of doll play provided positive feedback results as observed to determine a proven reduction in group isolation and discrimination. Yet, the length of this attitude change to continue or degree of attributed permanence this experience provided was declared unknown by the authors of this study but more important was to have established the change short term, which was a significant positive outcome in this experience. This empirical study led the pathway to other prejudice reduction activities in the form of anti-bias education and developed multicultural education curricula (Paluck \& Green, 2009). 
Nevertheless, Gimmestad \& De Chiara (1982) state that one shortcoming of many prejudice reduction programs in schools is their assumption that by increasing students' knowledge about other groups, they will eliminate any misconceptions or false stereotypes these students may hold about those groups. Although, knowledge can contribute to prejudice reduction, the authors assert that such programs will not reduce prejudice by itself. The authors make reference to Moreland (1963, as referenced in Gimmestad \& De Chiara, 1982), where this author contends that "Facts do not speak for themselves, rather they are interpreted through the experiences and biases of those hearing them" (p. 125)

\section{Physiology of Problem: A Conceptual Framework}

Qualitative researchers, specifically in academia of the social sciences, seek to gather a thorough understanding of human behavior and the reasons that govern such behavior (Denzin \& Lincoln, 2005). Therefore, the researcher is trying to learn about improving the school environment in order to find out how prejudice behavior can be reduced. According to Gephart \& Marsick (2003), the action research approach is best in getting to know more about the social or ecological system (McKown, 2005) in which we function while attempting to resolve problems within them along with building the capacity for learning and change (Freeman \&Vasconcelos, 2010; Gephart \& Marsick, 2003).

Furthermore, this conceptualization is structured in order to help practitioners understand what factors promote organizational changes in reducing prejudice behaviors and or practice. Therefore, educational leaders then may apply the leadership training skills in building a caring school environment to the efficacy of an enhanced leadership framework. In order to effectively learn about improving the school environment, the conceptual framework as illustrated in Figure 2-4 is an adapted concept map (Clark, 2004) that will 
look at what factors promote organizational change through effective informed practice.

The research study may then analyze how prejudice behavior can be reduced from a performance improvement perspective as well adapting the intervention strategy model of McKown (2005) in the form of a proposed leadership skills training paradigm model and continuum:

\section{Conceptual Framework}

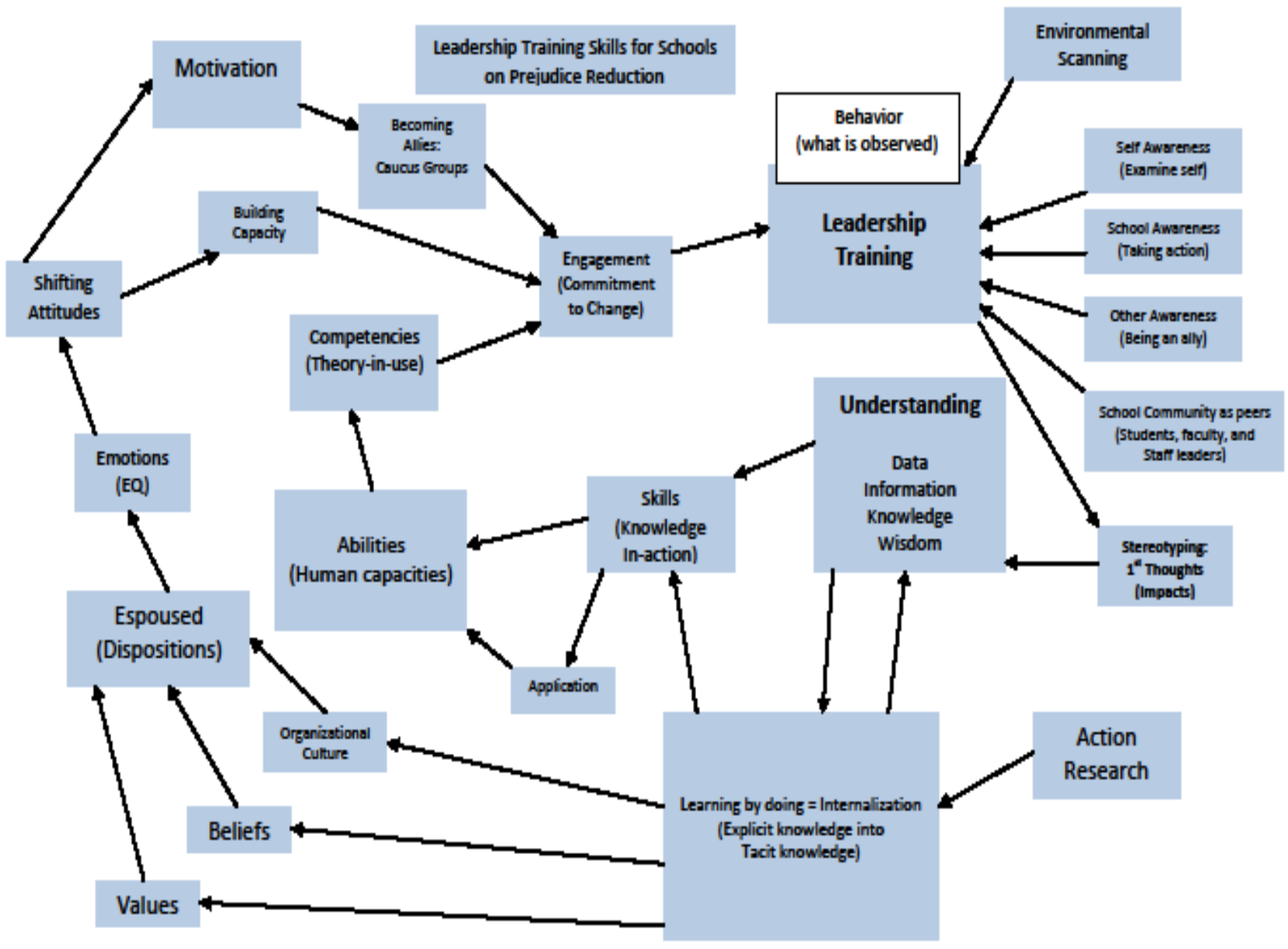

Figure 2-4 Conceptual Framework Map as adapted from Clark (2004) 
Therefore, based upon the conceptual framework of Brown (2009), and using the performance improvement mapping approach as adapted from Clark (2004), a conceptual framework map was designed by the researcher, incorporating the skills application training modules identified later in this chapter. Hence, the makeup of the researcher problem looks at capacity building for learning and organizational change (Brown, 2009; Epstein, 2004; Gephart \& Marsick, 2003) in order to effectively learn about improving the school environment. Specifically, the conceptual framework will provide insight on what factors or variables promote organizational change through effective informed practice (WillardHolt, 2000). Lastly, the researcher as participant-observer may then analyze how prejudice behavior can be reduced from a Performance Improvement perspective (Clark, 2004) in the form of a diversity leadership skills training paradigm model adapted from Brown (2009) and continuum plan; henceforth, embedded in a school improvement plan for suggested implementation.

\section{Evaluation of Multicultural Intervention}

According to Reed (1996), the author suggests that discrimination often occurs "when people believe themselves to be a superior based on their race, gender, socioeconomic status" (p.81) or some other private identity, hidden group. She asserts that the organizational framework of our schools in the United States "and the belief systems of educators can perpetuate such notions through educational programs, policies, practices, and processes" (Reed, 1996). To reiterate, it is imperative for school leaders to strategically reduce the conscious and unconscious discourses "that support prejudices and notions of superiority if all [students, teachers, and administrators] are to realize their relatively untapped potential"' (Reed, 1996, p.81). This self-concept theory, according to Purkey 
(1992) is important to helping one understand that "behavior is mediated by the ways an individual views oneself, and that these views serve as both antecedent and consequence of human activity.”

Contributions to existing literature in support to the proposed goals and objectives for prejudice reduction practices, particularly in urban education, are modeled after Haberman's (1994) five step approach to facing prejudices:

1) Analyzing prejudices: recognize the misinformation that people have learned about various groups

2) Seeking the sources of the beliefs: identify and heal from internalized oppression - the discrimination members of an oppressed group target at themselves and each other

3) Examining the benefits of prejudice: celebrate similarities and differences; claim pride in group identity

4) Considering the effects of prejudices: understand the personal impact of discrimination through the telling of stories, and

5) Planning to eliminate prejudices: learn hands-on tools for dealing effectively with bigoted comments and behavior.

Additionally, Purkey (1992) presents the five-level conflict-management process.

Reed agrees that this process adapted to prejudice serves as the five-part action plan in Haberman's fifth step as mentioned herein. According to Reed, utilizing collectively Haberman's model and Purkey's model create an invitational approach, called the Rule of the Five Cs (Purkey, 1992): (1) perceiving concern while listening to and repeating back each side's concerns; (2) conferring with oneself in order to formulate questions that elicit more information; (3) consulting with colleagues by welcoming personal stories that explain the underlying significance of each side's position; (4) confronting the crisis by means of recognizing the "emotional ring" in the issues presented; (5) combating prejudices through mapping out points of agreement as well as disagreement, and then reframing the issue in a way that allows the disputing parties to find common ground. 


\section{Reframing Organizational Change}

In order to assess the effectiveness and quality of a skills training's effectiveness on the impact of informed practice necessitates an appropriate plan of action. According to Bolman \& Deal (2003), reframing, like management and leadership, is much more art than science for igniting the order of change needed to target success in facilitating this action research project. Bolman \& Deal (2003) approach reframing organizations through the four frames as well as outline the barriers to change with essential strategies. The Structural frame's barriers to change entail an organization's loss of direction on clarity and stability that often leads to confusion and chaos. Essential reframing strategies suggest communicating, realigning, and renegotiating formal patterns and policies. Barriers to change in Human Resources frame would have anxiety as well uncertainty of the people in the organization who may feel incompetent and needy. However, essential strategies for reframing would include training to develop new skills. Engaging participation and involvement is essential to include some type of psychological support.

From a political standpoint, barriers would entail disempowerment with conflict between groups or rather conflict between the winner and losers within the organization (Bolman \& Deal, 2003). To effectively reframe political barriers, change agents would create arenas for negotiating issues and form new alliances. Finally, Bolman \& Deal maintain that symbolic frame barriers have change. These individuals within the organization are always clinging to the past. Successful strategies for reframing would suggest transition rituals or implementing a means to morn past yet celebrate future with changes to benefit the organization and stakeholders. 


\section{Organizational Change}

Kotter (1996) addresses this awareness assessment through his eight-stage process for organization change. He explains how his theory-in-use for successful school improvement planning and implementation will assist in promoting real change. To effectively address the research problem, the overall objective of school improvement planning is an enhanced level of student achievement through an enhanced leadership framework and successfully implemented program. The specifics of Kotter's change framework will be described in more detail in the methodology chapter.

\section{Salient Factors Impacting Prejudice}

The salient factors impacting prejudice can be learned through modules as applied for developing training skills and an inclusion leadership framework for schools that could be used to ameliorate the social justice issue (Brown, 2009). These analyzed areas of critical concern are presented all over the country in institute conferences on Leadership for Diversity through facilitated professional development trainings as adopted from the National Coalition Building Institute (Brown, 2009; NCBI, 2010 \& 2011). Specifically, as explained through a train-the-trainer seminar, student, faculty, and staff leaders are afforded the opportunity to come together to become more effective leaders and allies at their school. This program is unique in many ways. In that, the training modules bring together people from all parts of the school community as peers with equal status and provide practical skills that can be used in everyday situations to create a safer and welcoming school environment for all (Brown, 2009; NCBI, 2010 \& 2011). 
Moreover, Pate (1995) published an information analysis report on the evaluative and feasibility of prejudice reduction. Pate concluded in his report findings that some generalizations, which are offered, have the potential to lead towards a less prejudiced society. The National Coalition Building Institute (NCBI) Prejudice Reduction Workshop model (Brown, 2009; NCBI, 2010 \& 2011) reflects Pate's researched findings as summarized in the following categories:

1. the use of special materials;

2. cognitive approaches

3. director approaches;

4. shared-coping approaches;

5. various classroom approaches (i.e. sensitivity training, multicultural education);

6. audio-visual approaches;

7. consideration of school-wide conditions;

8. teacher training (i.e. Diversity Skills Training for Schools);

9. human relations training;

10. positive reinforcement approaches;

11. a variety of approaches; and,

12. social contact theory approaches

Pate concludes his 1995 research findings in summation, indicating that "in spite of the problems in research on prejudice reduction, there is a body of knowledge that suggests that, in working with young people, it is possible to reduce existing prejudice and even prevent its formation" by means of increasing a person's self-esteem, helping a person perceive others as unique individuals, and sharpening cognition skills are helpful in this process. This research was conducted over 15 years ago in 1995, it found that "genuine multicultural education shows great promise in prejudice reduction" (Pate, 1995). 
Likewise, Folger, Poole, \& Stutman (2001) argue that "Social confrontation episodes involve conflict over conduct and rules of conduct" (p.99). Misinterpretations and obscure behaviors complicate the relationship between various groups within the learning communities. These misunderstandings are the result of diversity in ethnic backgrounds; others are caused by widely adapted generational disparities. Brown (2009) \& Folger et al. (2001) assert that people who are out of touch with their feelings transmit many mixed messages.

Moreover, there are varied approaches to diversity skills training and prejudice reduction action taken as a result of supported leadership training skills for schools (Brown, 2009). Different situations require diverse decisions methods to satisfy the overall constituents and physical entities. This action taken in prejudice reduction and conflict management practices can be either constructive or destructive in the aspect of progress to the functioning of educational leadership in its controversial issues. Lessons learned in becoming more effective in this process would perhaps to research and analyze further how well groups in organizations use varied approaches in prejudice reduction.

\section{Leadership for Diversity: A Prejudice Reduction Model}

The NCBI Prejudice Reduction Workshop Model (Brown, 2009) presentation was introduced as a diversity initiative to the researcher in a Train-the-Trainer institute conference series. These institutes were attended to at the recommendation of school administration in order to assist in the reorganization process of the agency's early education programs. The researcher was then certified to lead and model the workshop trainings as adapted from the framework of Brown (2009). Brown states, "This model has 
been tested in thousand of settings over a 20 year period" (Brown \& NCBI, 2009, p. 50).

\section{Learning by Doing}

According to DuFour, DuFour, Eaker \& Many (2006) support the argument that learning by doing modeled practices as demonstrated through Brown's (2009) workshop models does show to believe that every group counts and every issue is important. In the conference institute proceedings, Brown stated:

"In these trainings, student, faculty, and staff leaders will have the opportunity to come together to become more effective leaders and allies at your school. This program is unique in many ways. It brings together people from all parts of the school community as peers and provides practical skills that can be used in everyday situations to create a safer and welcoming school environment for all” (Brown, 2009).

The idea is that in today's work environment, specifically within the learning community, people do not ordinarily get to interact with one another at the intersection of culture and their humanity (Brown, 2009; Craig, 2008; Watts, 1985). The modeled workshops will allow people to learn from sharing stories, relate, and reflect. Table 2-2 represents an attempt to identify diversity skills' application while building capacity. 
Table 2-2

Prejudice Reduction Workshop Model (Brown, 2009; NCBI, 2010 \& 2011)

\begin{tabular}{|c|c|c|}
\hline $\begin{array}{l}\text { Training } \\
\text { Module }\end{array}$ & Diversity Leadership Skills & School Application \\
\hline $\begin{array}{l}\text { Three Levels of } \\
\text { Leadership }\end{array}$ & $\begin{array}{l}\text { Knowing and practicing the three levels: } \\
\text { - Self Awareness - examine self } \\
\text { - Other Awareness - being an ally } \\
\text { - School Awareness - taking action }\end{array}$ & $\begin{array}{l}\text { Recognizing that in whatever roles we } \\
\text { have within the school, we can take } \\
\text { different levels of leadership to enhance } \\
\text { the school environment for ourselves } \\
\text { and others. }\end{array}$ \\
\hline $\begin{array}{l}\text { Environmental } \\
\text { Scanning: } \\
\text { Ups/Downs } \\
\text { Activity }\end{array}$ & $\begin{array}{l}\text { Respecting and welcoming diversity in all } \\
\text { forms - "Every Group Counts." } \\
\text { Asking: "Did we leave anybody out?" }\end{array}$ & $\begin{array}{l}\text { Noticing which school groups get } \\
\text { respect and which groups get excluded } \\
\text { and reaching out to them. }\end{array}$ \\
\hline $\begin{array}{l}\text { Stereotyping: } \\
\text { First Thoughts } \\
\text { Exercise }\end{array}$ & $\begin{array}{l}\text { Understanding how stereotypes impact our } \\
\text { actions and attitudes and how we can change } \\
\text { them. }\end{array}$ & $\begin{array}{l}\text { Examining the stereotypes within the } \\
\text { school that create tension and conflict. }\end{array}$ \\
\hline $\begin{array}{l}\text { Internalized } \\
\text { Oppression }\end{array}$ & $\begin{array}{l}\text { Learning the negative impact that stereotypes } \\
\text { have on our own and different groups' self } \\
\text { image (Purkey, 1992) and behavior. }\end{array}$ & $\begin{array}{l}\text { Exploring ways to "contradict" and to } \\
\text { interrupt the internalization of negative } \\
\text { stereotypes on all groups. }\end{array}$ \\
\hline Building Pride & $\begin{array}{l}\text { Encouraging the expression of pride in all } \\
\text { groups. } \\
\text { Understanding the difference between } \\
\text { authentic pride and chauvinism. }\end{array}$ & $\begin{array}{l}\text { Creating opportunities for students, staff, } \\
\text { and all groups to express pride and } \\
\text { receive recognition. }\end{array}$ \\
\hline $\begin{array}{l}\text { Becoming } \\
\text { Allies: Caucus } \\
\text { Groups }\end{array}$ & $\begin{array}{l}\text { Gaining new awareness and information on } \\
\text { how different groups experience mistreatment } \\
\text { and learning how to be better allies to them. }\end{array}$ & $\begin{array}{l}\text { Identifying school groups where there is } \\
\text { mistreatment and misinformation and } \\
\text { correcting it. }\end{array}$ \\
\hline $\begin{array}{l}\text { Making } \\
\text { Commitments to } \\
\text { Change: Speak } \\
\text { Outs }\end{array}$ & $\begin{array}{l}\text { Learning how different types of } \\
\text { discrimination affect individuals and groups. } \\
\text { Learn the power of the personal story and how } \\
\text { it can effect change. }\end{array}$ & $\begin{array}{l}\text { Determining the Speak Outs that need to } \\
\text { be told and heard in order to make the } \\
\text { school environment safe and welcoming } \\
\text { for all. }\end{array}$ \\
\hline $\begin{array}{l}\text { Shifting } \\
\text { Attitudes: Role } \\
\text { Playing and } \\
\text { Skits }\end{array}$ & $\begin{array}{l}\text { Preventing and interrupting prejudicial jokes, } \\
\text { comments, and slurs. Noticing and supporting } \\
\text { both the perpetrator and victim of prejudicial } \\
\text { jokes, comments and slurs. }\end{array}$ & $\begin{array}{l}\text { Identifying the most common situations } \\
\text { in school where individuals experience } \\
\text { teasing and put-downs and interrupt the } \\
\text { behavior and/or support the victims. }\end{array}$ \\
\hline
\end{tabular}


Additionally, Singh (1991) provides contributing agreement that prejudice may be reduced by "equal status contact between majority and minority groups in the pursuit of common goals." He further references that the effect is greatly enhanced if this contact is endorsed by institutional support (i.e. by law, custom, or local policy) and granted that it is an approach that leads to the awareness and appreciation of "common interests and common humanity between members of the two groups" (Singh, 1991).

Curriculum and instruction in a multicultural society is perceived as evidently a challenging task. According to Butt \& Pahnos (1995), teaching excellence and providing "equity in [urban] education is difficult to achieve when" members within the learning community have diverse "styles of communication, patterns of participation [in relation to learning disparities] and, views of the world" in relation to cultural beliefs and customs. Therefore, teaching and understanding the needs of every member within the learning community and taking action "to each one sensitively, fairly, and effectively" (Butt \& Pahnos, 1995) are essential in a multicultural education. The intention for curriculum and instruction should then have students emancipated from their cultural barriers by affording "a bias-free learning environment" (Butt \& Pahnos, 1995). Furthermore, it is clearly understood that the actual challenge for curriculum and instruction "is to make a difference in our students' lives; to help them understand that they are capable, unique individuals" (Butt \& Pahnos, 1995).

As educational leaders, we must place positive impressions on students learning by helping them to understand diversity in the school environment in which they learn. Through a successfully implemented prejudice reduction model program, school leaders are able to help structure how students perceive and interact within the learning community 
and society. Butt \& Pahnos (1995) conclude that a multicultural curriculum program affords diversity, because:

In its broadest sense, a curriculum influences, and is influenced by, the entire school environment. Curricula, therefore, must be viewed as all the experiences, both planned and unplanned, that learners are exposed to in school. Following this definition, a multicultural curricula, in its broad-est sense, embraces all diversity, ethnicity, race, and gender (Butt \& Pahnos, 1995).

The NCBI Prejudice Reduction Workshop Model is a useful resource tool for establishing and implementing a multicultural curriculum. Howard (1985) suggests his concept for prejudice reduction in urban education. Howard explains that multicultural learning should be addressed at three levels: head, heart, and hands. The head includes facts and information, the heart addresses attitudes and feelings, and the hands involve activities and actions. All three levels must be included in a comprehensive multicultural curriculum. All multicultural curricula should address five aspects: (1.) uniqueness entailing special qualities; (2.) empowerment in believing that you can do what you set out to do; (3.) belonging or feelings of being part of something; (4.) security in knowing rules will be enforced fairly and equitably; and (5.) purpose, whereby setting realistic goals and feeling challenged (Howard, 1985, as reference in Butt \& Pahnos, 1995).

Educational leaders can facilitate and model how students view and respond to the world by creating a welcoming learning environment that provides equity and reflects and embraces the diversity in which we live. A multicultural curriculum lays the foundation for a lifetime. In considering that this study's action research project was conducted in an early childhood education agency, best practices in early childhood education is suggestive to help students early on through exploring ways to "contradict" and to interrupt the 
internalization of negative stereotypes on all groups. Research shows that many children form racial biases as early as kindergarten (Gimmestad \& De Chiara, 1982; Tatem, 1997). The National Association for the Education of Young Children (NAEYC, 1995) supports the fact that the years from birth to age 5 are the most significant stage of a child's development. This information is supported through Tatum's (1997) extensive knowledge of developmental psychology along with citation as referenced from psychologist, William Cross.

Tatum successfully helps the reader understand the described stages of development: Pre-encounter, when young children simply absorb the messages they receive from those around them, not yet having reason to question them; Encounter, when an individual first becomes aware of racism through some "event or series of events that force the young person to acknowledge [its] personal impact" (p. 55); Immersion/emersion, when the individual works actively to learn about and affirm their own racial identity; and Internalization/commitment, when the individual has established a positive personal identity for him/herself.

On the other hand, early education scholars, particularly focusing on the area of child development theory, describe these similar stages of development comparatively to "what children do" (NAEYC, 1995) : 1 to 2 years, imitate adult actions [Pre-encounter]; 2 to $31 / 2$ years, act out familiar scenes [Encounter]; $3-1 / 2$ to 5 years, talk a lot (communicative), ask many questions; test physical skills and courage with caution; reveal feeling in dramatic play [Immersion/emersion]; 5 to 8 years, Grow curious about people and how the world works; gain more confidence in physical skills; use words to express feeling and to cope; Become more outgoing, play cooperatively; whereby, the child will 
engage in problem-solving, practice teamwork, develop sense of personal competency [Internalization/commitment]. Likewise, Tatum (1997) uniquely incorporates her understanding of developmental psychology through her own personally shared life experiences to investigate this issue of racial identity and integration. The author contends that people have a racial identity, and the first stage to prejudice reduction in society is accepting this notion.

Similarly, every child is an exceptional person with an individual personality, learning style, family background, and development of growth. However, the researcher believes as an early childhood education professional, that there are common, expected progressions of development and transition that take place within the first nine years of life. As children develop, they need diverse types of stimulation and interaction to apply their budding skills and to build on new ones. At every age, meeting basic health and nutritional needs is essential as well as the hope of understanding a sense of self or identity in a child's emotional environment (NAEYC, 1995).

Likewise, from birth and beyond, diversity exists in the cognitive processes for how an individual acknowledges and in time understands one's racial identity. As reviewed, Tatum (1997) outlines in her book the development of racial identity throughout an individual's stages of life, providing the reader further understanding that may perhaps have an internalized oppressive issue. Tatum explores for the reader in her book this racial identity process, both for Blacks and for Whites. Furthermore, if children emerge from this stage of their development with an unstable foundation, then families, schools, and society pay the ultimate price for their children: poor school performance, dropouts, and crime all 
tragically enough become much more likely. The key to saving children and breaking this terrible and consequently expensive cycle is to help them early in their lives.

\section{Discussion and Conclusions}

The literature review informed the researcher's action research design as well as identified the main aspects of inquiry. In addition, theoretical and conceptual framework approaches were discussed to include clarification on the research problem. Moreover, the literature review provided insight from previous investigative research to appropriately inform on the researcher's intent to address the research problem.

Future lessons learned are that the controversial issue process involving prejudice reduction and conflict management will tend to be situational specific in finding an integrative solution, and possible dispute outcomes will depend on the mutual interests and best alternatives met for the addressed needs of all group members. Moore states (1996) "Once a party has assessed its interests and those of other parties and reviewed potential dispute outcomes, it must select a particular approach to reach the desired end" (p104). We must then analyze the approaches to fully understand how these general procedures are applied in reaching a resolution for future empirical research on this current subject of academic consideration. Likewise, Brown's (2009) prejudice reduction workshop model is a useful training continuum tool in conjunction with McKown's (2005) strategic approach for implementation and as an intricate part of the school improvement planning process.

Once more, Brown (2009; NCBI, 2010 \& 2011) maintains that the model has been tested in thousands of settings over a 20 year period.

Lastly, it is anticipated that through this study, the learned research findings will continue to empower that dedication for the interaction of the urban school's learning 
communities and supported levels of leadership to engage them while using the Prejudice Reduction model. The researcher aims to provide validity through the collected data obtained to determine the higher the degree of participation, the higher is the chance of program success. This social justice issue research study is designed as reflected to assist in the support for implementation that urban education programs must eventually experience. 


\section{Chapter 3}

\section{Methodology}

This chapter describes the research questions and methodology used to conduct the action research study. The research questions, description of data collection method used, the survey instruments, participant description, and research paradigm are covered in this chapter. Furthermore, other integral components of this chapter include identification of the research setting, subject participants, the conceptual, theoretical, and change framework approaches, overview of action research cycles, data analysis as well as role of the researcher, and conclusions drawn on informed action. Lastly, the information obtained for this action research study, using data from compiled surveys/questionnaires, pre/post assessments, observational and interview protocols (Creswell, 2007), was compared with existing research relating to other application skills intervention strategy approaches with similar representative samples.

This research study was designed to help measure the effectiveness the diversity leadership skills training could have in enhancing capacity building strategies through individual and professional development initiatives to help reduce prejudice behavior to improve the delivery of a positive educational program. Additionally, the physiology or the makeup of the research problem explored capacity building for learning and organizational change (Brown, 2009; Epstein, 2004; Gephart \& Marsick, 2003). My direct involvement as participant observer afforded me the opportunity to see positive change among the participants during the course of their eight week diversity leadership skills training modules. 


\section{Primary Research Question}

This study sought to answer the following research question along with possible subsidiary questions:

How does diversity leadership training skills on prejudice reduction result in changed beliefs of discriminatory behavior in schools?

\section{Subsidiary Analytic Questions.}

1. What are the effects of informed practices on organizational attitudes and behavior outcomes of education?

2. What factors promote changes in reduction practice of prejudice behavior in schools? What factors impede these changes in reduction practice of prejudice behavior issue schools?

3. Based on the data collected from the findings, how can the diversity leadership training be strengthened for the organization?

\section{Methods}

Human subjects were recruited to participate in the action research study, and the researcher obtained consent for survey. In addition, the researcher reviewed the required informed consent with all participants (Morgan, 1997) in part as an orientation and introduction to study. Observational protocol methods (Creswell, 2007) were used on participants during the eight week module activities in considering the use of a minimal group paradigm approach (Rubin, Hewstone \& Voci, 2001). Each participant was issued a certificate of completion at the conclusion of eight week training (See Appendix J).

Use of the Rubin, Hewstone \& Voci approach (2001) in action research by randomly assigning study's subjects into diverse groups, afforded participants' shared 
learning opportunities during the module exercises. As a result, research participants were engaged in the study's facilitated workshop activities as well as were able to observe and reflect on their group experiences.

Consequently, in order to best facilitate my research with the aim to answer research questions, I implemented a variety of triangulated instrumentation approaches in the process of my research design, data collection, and action research cyclic process: A conceptual framework approach, using Clark’s (2004) performance typology mapping technique provided insight on discovering what factors or variables promoted organizational change through effective informed practice (Freeman, 2010; Freeman \& Vasconcelos, 2010; Willard-Holt, 2000). The theoretical framework of McKown (2005) was adapted from his strategic planning model based on ecological theory, and it was used in conjunction with the adapted concepts from the prejudice reduction workshop model of Brown (2009).

McKown's model suggests that alterations at one or many levels of the ecology can lead to changes in individual behavior. Therefore, using his strategic planning model from the author's perspective has many consequences for intervention. Additionally, I found that McKown's approach paralleled Brown's workshop model in which I successfully modeled the prejudice reduction strategies of Brown (2009). Activities observed included (a) participants establishing plans to develop caucuses for allied group work; (b) shared time for speak outs in efforts for making commitments to change; (c) shared storytelling and reflections; (d) creative works with role playing and skits with participants for effective shifting attitudes. In addition, the participant learning reflections were valid uses of data to collect as part of the study's feedback process. This method according to DuFour, DuFour, 
Eaker, \& Many (2006) demonstrates the ideology of learning by doing. Therefore, action research served as the conduit for the researcher to facilitate the organization's professional development training.

In addition, DuFour et al. (2006) maintain that capacity building initiatives simultaneously incorporate intervention activities (McKown, 2005) such as: (a) ecological assessment (pre assessments, interviews, observations) to determine what structures and process in ecology or in the analysis of interactions among individuals contribute to prejudice; (b) selecting what interventions fit needs identified in ecological assessment; (c) using stakeholders, resources, and strategies used from Brown's adapted workshop modules to ensure intervention fidelity; (d) summative evaluation through learning reflections, and post assessments formatively to measure whether the intervention have the desired impact. The combined approaches were all incorporated and demonstrated in alignment to Kotter's (1996) change framework example.

This participatory action research (Freeman \& Vasconcelos, 2010) was carried out within the context of the action researcher's environment (Watts, 1985; Craig, 2008). In that, the action research study conducted was implemented in the learning environment by means of evaluation and inquiry (Freeman \& Vasconcelos, 2010) whether being participatory, engaging stakeholders themselves in identifying and naming injustices or forms of oppression present, and pedagogy by means of assessing practices as well as action oriented, as in the researcher's action research study, in order to establish the development of new understandings and behaviors. Craig (2008) defines action research as a process in which participants examine their own educational practice systematically and carefully, using the techniques of research study. Therefore, the evaluation and inquiry 
approach is contingent on changes in practices and material conditions that, according to Freeman \& Vasconcelos (2010) cannot rely on rhetoric alone.

The researcher facilitated the professional development trainings' module activities and diversity workshop models. My primary goal was to make an attempt in assessing the program participants' continued success for the efficacy of its diversity awareness training and to model change. Inasmuch, the researcher's goal was to encourage continued support within the organization's learning communities (DuFour \& DuFour, 2010; Kotter, 1996).

As a change agent, this role afforded me the opportunity to serve in the role of researcher as well as educational consultant to the educational agency, leading the study and the eight week diversity leadership training skills workshop modules. Furthermore, all information that can identify the school or representative(s) was coded and kept securely stored and locked in a cabinet. Only the researcher had access. Likewise, the participants' confidentiality was secured by using the online tool, Survey Monkey, and pseudonyms for focus group members at the conclusion of the study's findings' written analysis. Lastly, at the end of the study all information that could identify the center or representative(s) was destroyed. Only demographic information on the agency is matched with results.

Moreover, my intention in using the action research cyclic process was to present and explain all developed concepts included in the skills application model along with all aspects of the compiled baseline data from pre assessments and comprised analysis of the supporting triangulated information. Once the final coded data and research were compiled for analysis, a report of the findings was submitted to the central office administration as a consulting reference document. This progress report would later be used for seeking professional development funding resources as part of the agency training plan and 
collective inquiry format (DuFour et al., 2006) for the agency's proposed school improvement plan.

According to DuFour et al. (2006), the focus of collective inquiry is both a search for best practice for helping all students learn at high levels and an honest assessment of the current [educational agency] reality regarding teaching practices and student learning. The dialogue generated is intended to result in the academic focus, collective commitments, and productive professional relationships based on the researcher's capacity building module activities that enhance learning for teachers and students alike. The identification of further study in regards to this proposed recommendation and on informed practice is discussed in Chapter 5.

\section{Reliability and Validity}

Reliability and validity in this research design was enhanced by eliminating any subjectivity or potential biasing factors through the use of triangulation (Golafshani, 2003; Mills, 2003 \& 2011). This effort was practiced during surveying (pre and post assessments), observations and interviewing, assuring that the information obtained was dependent on controlled factors without any negating distracters. Therefore, the combined methods, working together, produced a complete action research study. Consequently, the study was defensible of its concluding findings as presented in Chapter 4 to include recommendations for further research on this topic discussed in Chapter 5 in considering proposed implementation for a school improvement plan.

\section{Rationale for Action Research}

According to Gephart \& Marsick (2003), the action research approach is best in getting to know more about the social system in which we function while attempting to 
resolve problems within that system. Moreover, qualitative researchers, specifically in the academia of the social sciences, aim to gather a thorough understanding of human behavior and the reasons that govern such behavior (Denzin \& Lincoln, 2005). Thus, I am trying to learn about improving the school environment of prejudice behavior, because I want to find out how prejudice behavior can be reduced. Action research always tries to solve problems.

In addition, the rationale for this type of research was appropriate in its process. Watts (1985) \& Craig (2008) define action research as a process in which participants examine their own educational practices systematically and carefully with others as change agents, using the techniques and strategic approaches of the research study. Watts (1985) further points out action research's appropriateness based on the belief that teachers and administrators work best on problems they have identified for themselves, and they become more effective when encouraged to examine and assess their own work, then consider ways of working differently. Likewise, this process of action research provides teachers and administrators the opportunity to help each other by working collaboratively (Mills, 2011). Whereby, "working with colleagues helps teachers and [administrators] in their professional development" (Watts, 1985, p. 118). To reiterate, the researcher's primary goal and purpose was to make an attempt in assessing this project's impact as well hope for its success in the continuum for the efficacy of its diversity awareness training and to model and encourage continued support within the organizational framework.

\section{Research Design}

The researcher used the paradigm perspective (Creswell \& Tashakkori, 2007) in the form of action research. Hence, this study was carried out within the context of the researcher work environment (Watts, 1985; Craig, 2008). As the participant observer, I 
used a process of systematic inquiry that was cyclical and conducted the study within the organization, rather than having the organization outsource an outside expert as consult for professional development training and education resource services.

As the consulting head teacher of the early childhood education program, my professional role served to conduct staff development, observe the center's program, and ensure the development and implementation of the center's early childhood development and activities program for children below six years of age. Overall, responsibility was to ensure the appropriateness of program activities according to both the age and developmental level of the child, as specified in N.J.A.C. 10:122-6.1(a) as well as provide administrative support to site administrators. As a result, facilitating the study as participant observer was an afforded opportunity for the researcher as well as the organization. Again, I was looked upon as a trusted insider who understood the agency's actual reorganization realities and vision as well as shared its private knowledge of organizational behaviors. For the purposes of doing this action research, the role as participant observer serves as a dual role within the organization and research study in which I conducted a research study while using a variety of strategies to collect data over the length of the study (Mills, 2011;

Wolcott, 1988).

\section{Contact Hypothesis}

Mills (2011) explains that the objective of traditional educational research is "to explain, predict, and/or control educational phenomena" (Gay et al., 2009, p.6 as quoted in Mills, 2011, p. 3). The hypothesis or the researcher's educated guess is a statement made by the researcher that explains what the outcome of the study will be. Whereas, the researcher's contact hypothesis in the case of this action research study aims to explore 
whether intergroup contact along with capacity building strategies reduces prejudice, discriminatory behaviors and attitude dispositions (Pettigrew \& Tropp, $2000 \& 2006$ ).

Moreover, participants were able to do shared learning exercised through the paired and grouped professional development training activities. The intent of the prejudice reduction strategies modeled was to demonstrate and then assess a measurable impact on skilled learning and application over time by the end of the action research study. These variables or skills included, but are not limited to, learning and recognizing the three levels of leadership, learning the significance of environmental scanning, discover how stereotypes impact actions and attitudes and how to change them, explore first thoughts through generated exercises in regard to particular groups, examine intragroup prejudice (internalized oppression), building pride and capacity, caucus reporting, learning the power of the personal story and how it can effect change by the use of speak out activities, and shifting attitudes through the use of role playing and skits (Brown, 2009; Gephart \& Marsick, 2003; Haberman,1994).

The ultimate outcome and expectation of this study's change initiative was to increase positive behavior as well as organizational awareness (DuFour, DuFour, Eaker, \& Many, 2006; Kotter, 1996). My expectation of the participants was to acquire a better understanding of prejudice reduction strategies as demonstrated through observational protocols (Creswell, 2007) and to ascertain that understanding from shared reflective learning assignments (Compton \& Davis, 2010) along with the post assessment training surveys. The use of multiple sources of collected data had enriched the study (Golafshani, 2003; Mills, 2003 \& 2011). In addition, Mills (2011) supports the triangulation principle with the use of multiple sources. He explains that "the strength of educational research lies 
in its triangulation, collecting information in many ways, rather than relying solely on one" (Wolcott, 1988 as referenced in Mills, 2011, p. 92).

\section{Research Preliminary Activities}

As the consulting head teacher for the early childhood education program, the action research project was deemed appropriate in accomplishing professional development goals for educational personnel and administrators during the summer work schedule. Training activities were mapped out, beginning with planning meetings in June to discuss the scope of the researcher's study that entailed an eight week summer training module series.

Prior to commencing the study, a letter to the HR Administrator to request initial permission through the Executive Director's office was sent, and a response was received for Board approved authorization (See Appendix A). After which, a written letter was sent to central administration explaining the purpose of study and requesting permission to solicit participation from each site location's center director. This letter of permission is a requirement of the Institutional Review Board (IRB) for Human Subjects Research at Rowan University. Choi (2010) explains that "[d]uring the data collection process, the ethics of research using human subjects [are] considered (p.77). I considered the two main issues that Choi addressed: informed consent and the protection of informants. Bogdan \& Biklen (2007) assert that "these issues attempt to insure that (1) informants enter research project voluntarily, and (2) informants are not exposed to risks greater than the gains they might derive" (p.77). Included in the initial communication to the central administration, the letter I sent explained the purpose of study and request for permission to solicit 14 
human subjects, representing participation from within the educational agency: The Board (1), Central Office (2), Site Supervisors (4), and Instructional staff (7).

After receiving the central administration's letter of permission, the researcher then followed up with the supervising representatives of each site through the technical support provided by the HR Administrator, corresponding with them via electronic email. At that time, the researcher detailed all procedures with the participating centers' directors with them prior to the survey and recruitment for the volunteer focus group interview and training session(s). With permission granted from the Board Chairman, the researcher commenced recruitment of volunteer participants through the assistance of a central office administrator. In order to obtain informed consent and consent for survey (See Appendix B), an initial information session meeting was held in collaboration with Central Office administration (June 2012). This meeting served as an orientation and introductory session for those participants recruited for introduction to study.

The orientation facilitated by the researcher was the opportunity to obtain the participants' informed consent that explained (a) an explanation of the purposes of action research; (b) procedures to be followed during the study; (c) identification of those procedures; (d) amount of time required for participation; (e) benefits of eight professional development hours issued to the participants for their voluntary time and cooperation in the study; (f) confidentiality of records and participants' responses maintained by the researcher; (g) a description of any reasonably foreseeable risks or discomforts to participant while engaged in the eight week training module activities; (h) no penalty or loss of benefits to participants should they discontinue participation at any time for clock hours not completed; (i) the researcher's contact information to the participants for 
questions related to the research; (j) review of participant's rights in accordance to Rowan University Institutional Review Board for the Protection of Human Subjects.

Lastly, the consent for survey (See Appendix B) was an information statement issued to all participants, explaining that surveys can be accessed online via Survey Monkey. This alternate consent statement explained (a) researcher as the principal investigator and what researcher was investigating; (b) procedures to be followed for completing surveys during the study; (c) by using this commercially available Survey Monkey tool responses will be kept anonymous and confidential via the Internet; (d) all participation was voluntary; (e) participants need not respond to all questions with no penalty to them; (f) the researcher's contact information to the participants for questions related to the research; (g) consent of all participants to conduct the study was authorized and approved by the Rowan University Institutional Review Board (IRB) for Human Subjects Research. IRB application's protocol was approved on September 28, 2011 (See Appendix C).

In addition, recruited participants were asked at once receiving consent for survey if they would kindly participate in a focus group study to span over an eight week period to which 14 subjects agreed to participate. At the commencement of the study, participants were administered the individual attitude pre assessment online survey questions (See Appendix D). The post assessment survey was the same instrument as the pre assessment survey. The participants electronically submitted online reflections and evaluations, including the six month follow up survey (See Appendix L) were completed within and at the conclusion of the study respectively. 
During the interview process and observational protocols (Creswell, 2007), each informant's willingness to participate in this study was witnessed using a consent form (Choi, 2010). The researcher stressed that participation was completely voluntary. In addition, eight (8) clock hours of professional development were offered as an incentive to focus group participants. According to Kotter (1996), he maintains that by generating short term wins as such in planning for visible improvements in performance, professional development as well as shared learning opportunities are essential for organizational change. Thusly, the eight (8) clock hours produced even more interest and motivation for participation in the study.

\section{Data Collection Strategies}

Action research comes from both qualitative and quantitative paradigms, but my research study was drawn mostly from qualitative embedded methods to include interviewing and then drawn upon some quantitative data to measure frequency and quality through the eight week professional development training workshops, observations, evaluation and assessment (asynchronous and synchronous). Statistical significance, however, was not applied as a measure for this study. Additionally, the use of interview protocol methods for the central office school administrators and site supervisors were helpful in the data collection process.

Specifically, observational protocol instrumentation was used during the module activities with the intent to monitor behaviors on impact of training application as well as validate other collected data (Creswell, 2007; 2003). The observation protocol was beneficial in measuring quality and effectiveness of workshop model training activities. Furthermore, the data obtained was coded to categorize participants' comments about 
diversity leadership skills and school application. These categories were based on feedback examples collected by the researcher. Hence, triangulation effectively facilitated validity in using the multiple sources of data from surveys, interviews, observation protocols, Likert attitude scales, and reflective journaling. As a result, trustworthiness and credibility were established.

The goal of this action research design, using qualitative data collection strategies for study, was to establish whether or not the findings address answering the overarching research question: "How does diversity leadership training skills on prejudice reduction result in changed beliefs of discriminatory behavior in schools?" Secondly, the goal of this action research design was to confirm the contact hypothesis that is implied on informed practice through peer training and professional development activities. The objective is to develop a continuum of activities with "the necessary enabling infrastructures that assist in the transference of knowledge gleaned from the training to concrete activity and practice" (Spiotta, 2004, p. 15). This study takes on the understanding that prejudice is learned and "that prejudice and interpersonal function can be changed through education" (p. 15).

\section{Coding Data}

The data collected were compiled and coded, the observational and research interview protocol data were collected, and the information was then reviewed, creating a coding scheme (Auerbach \& Silverstein, 2003; Creswell, 2007). These particular data coding strategies were successfully facilitated during professional development activities by the researcher as well transcribed from interviews to categorize participants' comments about diversity and the observed impact on participants from workshop trainings (See Appendix G, H, I). 


\section{Instrumentation}

On the basis of the instrumentation used by the researcher, organization and coding data (See Appendix G, Appendix H, Appendix I) were expected to allow identified themes to emerge from the measured data collected. To answer the research question on how does diversity leadership training skills on prejudice reduction result in changed beliefs of discriminatory behavior in schools, Instrument \#1, participant pre assessment (See Appendix D), and Instrument \#2, participant post assessment (See Appendix E) were developed and created online by the researcher, using Survey Monkey along with uniform methodology as modeled by Brown (2009) \& National Coalition Building Institute (NCBI, 2009). The online pre and post assessments each contained ten (10) Likert scale based attitude questions in measuring frequency: 5=Almost Always, 4=frequently, 3=Sometimes, 2=rarely, and 1=Never. Additionally, Instruments \#1 \& 2 were helpful as well in answering Analytical Research Question 1, "What are the effects of informed practice on organizational attitudes and behavior outcomes of education?

These two assessments were administered to participants through online link access during facilitated workshops (pre assessments) and then individuals were given two weeks to respond online, providing post training feedback (post assessments) based on submitted shared learning reflections via Survey Monkey, Instrument \#3 (See Appendix F). Instrument \#3 was constructed by the researcher along with reflective adaptations from NCBI (2009). The online participant learning reflection survey contained nine (9) open ended questions that allowed individuals an actual written response as an opportunity to provide their shared thoughts, experiences and feedback on the impact of diversity leadership skills training received. 
In addition, the learning reflection survey contained one (1) Likert scale attitude question, rating overall study significance and importance for diversity training: 5Essential, 4-Very Important, 3-Somewhat Important, 2-Neither Important or Not Important, 1-Not Important. Furthermore, Instrument \#3 in addition to the data collected from Instrument \#6, observational protocol (See Appendix I), were designed and specifically constructed for this study in use with Brown's (2009) prejudice reduction model together with Clark's (2004) adapted performance improvement conceptual mapping approach to answer Analytical Research Question 2, "What factors promote changes in reduction practice of prejudice behavior in schools? What factors impede changes in reduction practice of prejudice behavior in schools?

Instrument \#4, Central office administration interview protocol (See Appendix G), and Instrument \# 5, Site administration interview protocol (See Appendix H) were designed and constructed by the researcher, specifically for this study to: (a) to reflect the beliefs and attitudes on the mission and vision of diversity from the perspective of central office administration and on site school administration; (b) collect data from coding scheme to agency interview protocol transcripts in answering the third subsidiary question as well provide support to the primary research question, "How does diversity leadership training skills on prejudice reduction result in changed beliefs of discriminatory behavior in schools?"

Finally, a six month follow up online survey, Instrument \#7 (See Appendix L) was done (February 2013) to ascertain what module skills participants found useful in their work as well as which areas of skills application had they shared with colleagues since training. Confidentiality was always maintained by using this commercially available 
Survey Monkey tool that allowed the participants surveyed to anonymously reply via the Internet. Again, participants were asked to respond online. Three follow up survey question were designed and constructed by the researcher: Two of the questions, participants were given the option to select multiple choices from the survey's multiple answers. These survey questions were, "Which module(s) skills have you found useful in your work since you came back from training?" and "Which areas of skills application have you shared with colleagues?" The third survey question used by the researcher was a Likert type scale to measure likelihood with the following choices: 5=To a Great Extent, 4=To a Considerable Degree, $3=$ Somewhat, $2=$ Very Little, and 1=Not at All. This question asked, "Do you feel as though the training content helped you improve your ability to make decisions about the appropriate course of action involving prejudice behavior?"

The data collected was voluntary completed by all participants included in the study, and responses were stored confidentially and securely online by Survey Monkey. The researcher used unique identification codes to link respondents' answers to the information collected during the time of the eight week training as well as assured all respondents that this code system would not be used to identify them by name or obtain other information about the respondent. Conclusions and implications in regards to measuring post training impact as well as reflective practice are discussed in Chapter 5 .

The Survey Monkey tool in addition to data collected from the interview protocols were administered as the most appropriate valid method to gather collected data for two reasons: accuracy and efficiency. This chosen data collection strategy allowed the researcher to obtain immediate feedback; whereas, Instruments \#1 and \#2 allowed respondents to provide immediate written feedback. Likewise, the assessment survey 
instruments were designed to take less than five to ten minutes to respond. The survey method was tested to determine response times and ease of administration. These preliminary activities took place during the initial information session meeting held in collaboration with Central Office administration (June 2012). This meeting also served as an orientation for 14 people who volunteered to be participants for the study.

\section{Participants}

In response to the summer professional development needs communicated from my former supervisor, the Human Resources Administrator, I started the planning process of participant recruitment in June 2012. Interviews were conducted with program and site administration members. Sample participants were comprised of site supervisors, teachers, paraprofessionals, a Board Member, and central office administration. Additionally, the identities of all interviewed education administration program members, to include a Board member, who agreed to participate in an interview were known only to the researcher, thus kept confidential. A model presentation of the overall purpose and scope of study for the research project was conducted with a founding Board member and the Executive Director present.

Recruitment of subjects was solicited with buy in from those who volunteered to be issued eight professional development contact hours at the conclusion of study's training activities. This process of recruitment captured participants from representatives of Board members, central administration members, educational site administration, teachers, and teacher assistants was best to insure that all viewpoints were adequately represented from the purposeful sample obtained. The participants recruited and obtained by the researcher resulted in having respondents at both ends of a spectrum to include novice educators as 
well as some in the middle of whom had the best knowledge and experience. Action research project activities commenced July 9, 2012 each week consecutively on site at an inner city facility location. The scope of project's summer activities ran for eight consecutive weeks to include six month follow up survey (See Appendix L) administered in February 2013.

\section{Background of Organization}

A mission statement or an organization's formal business statement of purpose is a formal document that states the program goals of a company or organization. Starling (2002) states that a "mission statement brings the agency into focus. [The mission statement] explains why the agency exists, tells what it does, and describes how it does it" (p. 208). The 1969 mission statement read "to promote positive self-image and quality Child Care for children, as well as, encourage Parental and Community Involvement" (BPUM Parent Handbook, 2003, p.1).

Today, the mission statement is "to provide quality Early Child Care and Education to infants, toddlers, and preschool children in the City and County. The prime purpose of this program is to: 1) develop and support students to assure high levels of achievement, 2) promote an efficient and comprehensive curriculum reflective of the political, social and cultural aspects of society, 3) promote a positive self-image in our students, and 4) increase

and expand programs that encourage greater parental and community involvement" (Board Member interview, 2012).

With this clear understanding of mission and the agency's desired outcomes of the strategic management process, throughout its history, the "program has established a tradition of responsiveness to the needs of the community (our customers), excellence in 
programming (implementation) initiatives, and most importantly in educational and organizational leadership for advocating of children and families" (Board Member interview, 2012).

\section{Services locations to the community at large.}

Through its four (4) day care centers, two in the City, and two in the County, the two City centers are NJ approved Abbott School District satellite centers that operated a school-age program in conjunction with the City's public schools. The agency provided educational enrichment and maintained Board of Education classrooms for 3- and 4-year olds. Through the BOE program, the agency is able to provide Health and Social Work services by the employment of a School Nurse and a Family Services/Social Worker. The follow provides brief background histories on each center location:

West

In 1975 the demand for child care services increased rapidly. Therefore, the agency initial program was expanded to the West location in the City. The program offers comprehensive child care services to pre-school children whose parents are employed, in training, and those who are seeking to further their education in college. In order to maximize on the full use of facilities, the founding agency's initial program facility was merged into the site at this location. This Center includes the Board of Education program.

\section{County ( $1^{\text {st }}$ location $)$}

In 1979, the agency became aware of the evident need for child care services in the County area. At that time, the agency opened its third set of doors. This location is licensed to serve 60 children and their families. 


\section{South}

In 1982, the agency merged with the City's Children's Center. This merger allowed program expansion of services to infant and toddler care. Through the merger in 1982, the number of employees grew to 47 and the number of children served is 244 . This Center included the City Board of Education program.

\section{County College location}

As of August 2006, the agency was the successful vendor to provide child care at the local community college. The county location provided on-site services in the priority of children of college students, children of faculty/staff and children of community members. Subsidized child care assistance is available to those eligible parents. Also, this location accommodated the Work First New Jersey (W.F.N.J.), New Jersey Cares For Kids (N.J.C.K.) and other state voucher related programs.

\section{Setting}

In order to assess participant dispositions and the efficacy of diversity leadership training, this research project targeted an early education agency whose founding program goals in March 1969, were to benefit the needs and concern African American population to now a diverse multicultural and multiracial community population of children and families.

For over 20 years, this educational, social, and communal agency's founding mission and vision were primarily focused on the Black community. At the time of this agency's incorporation, the early education program services were meant for the African American community and everyone within and outside the agency understood this fact. There were few dedicated educational services for the Black community at that time, and 
these services were truly needed in the City of Camden. However, the site locations and service population in the community had changed over the years, as well as communal and social attitudes concerning race. Another reason for greater racial and ethnic enrollment was the provision of better services, outreach to the community, self sufficiency, and financial assistance.

The child development program was formed in order to address the educational and comprehensive childcare needs in Camden City. The early education program's parenting entity, the Black People's Unity Movement Impact Corporation, was located in a community that is economically, linguistically, racially and ethnically diverse. The founder of the agency's economic development corporation, Charles R. "Poppy” Sharp (19691999), an activist for the Black community in The City of Camden along with his colleague, an attorney, who was the Executive Director at that time. These two individuals were the driving forces behind the development of the day care program.

Moreover, the parenting entity of the child care agency was once named one of the largest county employers (Davis, 1988). In addition, in 1988, the organization was honored by the local County vocational and technical high school (later renamed as a county technical school) in recognition and appreciation for services as a Co-operating Training Station for students, namely the researcher at that time, enrolled in the local County Vocational and Technical Schools' Co-operative On-The-Job Training Program.

The researcher's involvement with the program started November 30, 1987. The progression of my professional involvement with the program developed over the next 25 years. Past roles I have served were: (a) Receptionist/Clerk Typist for Central Administration; (b) Personnel Office Assistant; (c) Child Day Care Center Administrative 
Assistant; (d) Enrollment Specialist; (e) Head Teacher; (f) Professional Development Specialist; (g) Interim Administrative Assistant Director; (h) Consulting Head Teacher. As a result of this professional relationship, the researcher had access to and the confidence of the agency's administrators, Board Members, as well as teachers and staff. I was looked upon as a trusted insider who understood the agency's actual mission reorganization facts and shared its private knowledge of organizational behaviors.

\section{Change Framework}

In order to assess the quality of the study's training effectiveness on informed practice, the study necessitated an appropriate plan of action for change. The change framework aimed in its attempt to primarily focus on enhancing the learning environment, the school leadership framework, building capacity and effective coalitions; whereby, ultimately improving the level of student achievement. It is with this intention that the researcher attempted to demonstrate understanding of the paradigm frameworks on organizational change. A description on specific actions taken as designed in a proposed plan for delivery of change would allow and support recommendations about a training plan of action and serve as professional development for the agency's employees and Board members is described in Chapter 5. The priority area of focus for this plan was to target success at reducing prejudice behavior and conduct in the school environment.

According to Bolman \& Deal (2003), the authors explain that reframing, like management and leadership, is much more art than science. The authors maintain that reframing for initiating the order of change is needed to target success in facilitating this action research project. Additionally, the authors assert that organizations are focus oriented. The essential strategies for reframing organizational change entail 
communicating, realigning, and renegotiating formal patterns and policies within the internal and external structures. Additionally, the Human Resource framework aspects for these essential strategies include the researcher's training of the participants to develop new skills.

Accordingly, Fullen (2001) supports the concept of tacit knowledge in which these new skills are applied through participation and involvement in reframing organizational change. In addition, tacit knowledge entails the skills, beliefs, and understanding that are below the level of awareness. As a result, the sharing of tacit knowledge among multiple individuals with different backgrounds, perspectives, and motivations becomes the critical step for organizational knowledge creation to take place (Fullen, 2001). Moreover, Fullen further supports this framework in which establishing knowledge sharing practices is as much a route to creating collaborative culture as the organization must frame the giving and receiving of knowledge as a responsibility. The organization must reinforce such sharing through incentives and opportunities to engage in it. Therefore, the logic of this sharing practice is that individuals will not engage in sharing unless they find it motivating to do so. This implication is based upon whether because participants feel valued and are valued, because they are getting something in return, or because they want to contribute to a bigger vision (Fullen, 2001).

Lastly, Bolman \& Deal (2003) explain that the Human Resource frame provides the psychological support where training, participation and support can increase understanding of why change is needed, as well as skills and confidence needed in the school implementation process. Thus, the participation in the diversity leadership training and the practiced skills application take aim in targeting towards the reduction of prejudice 
behaviors and conduct. As a result, the researcher suggests that an enhanced leadership framework would be in alignment with the agency's mission statement to promote positive self image and quality education for students as well as encourage community awareness and involvement at all levels.

\section{Researcher's Formulation of Personal Theory}

Consequently, to effectively communicate the change vision (Kotter, 1996), the researcher's proposed change theory model diagram presented in Figure 3-1 for practicing prejudice reduction skills, illustrates how the researcher's personal theory (Compton \& Davis, 2010; Knowles \& Cole, 1996; Mills, 2011) to include the identified dependent variables: Three Levels of Leadership, School Community as peers and caucusing (becoming allies) are all suggestive when considering targeted success in the amelioration of prejudice behaviors while successfully enhancing leadership framework in respecting diversity.

\section{$\underline{\text { Arrow Diagram and Model }}$}

Brown's Prejudice Reduction

Model (2009)

Three Levels of Leadership 1. Self Awareness - examine self

2. Other Awareness - being an ally

3. School Awareness - taking action
Model: A simplified version of a theory that captures its key components and is amenable to empirical testing.

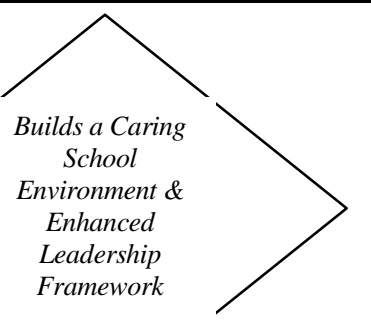

(Including student, faculty, and staff leaders)
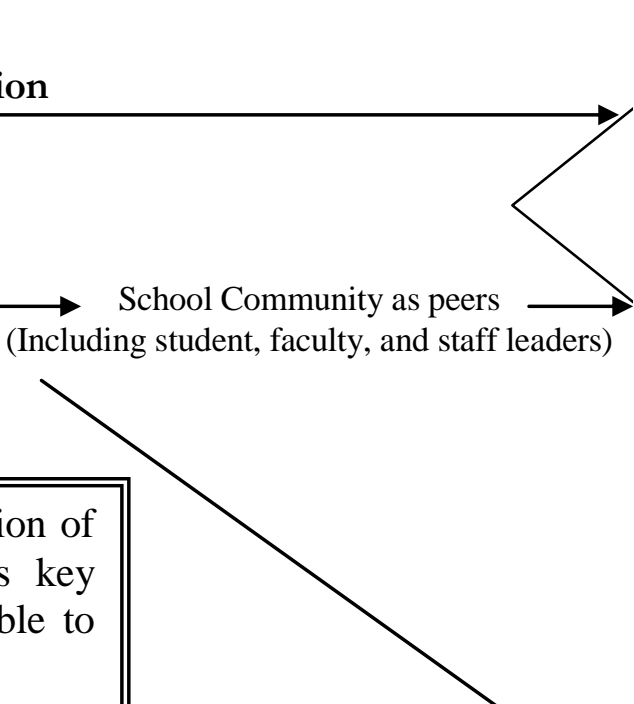

Framework

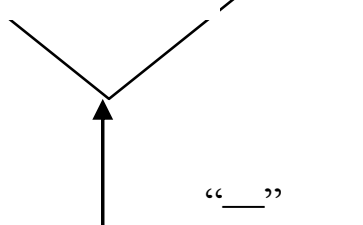

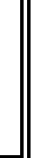

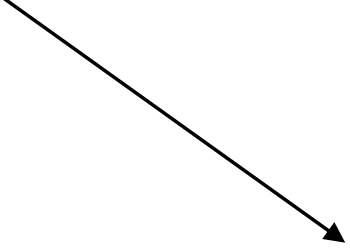

Non-Participation

Figure 3-1 Researcher's Proposed Change Theory Model 
Figure 3-1 designed and created by the researcher for this study proposed a change theory model conducive to teaching and learning as this relates to this research project with the intention of influencing future practices:

1. The greater application uses of the model, the greater the chance of a safer and welcoming school environment for all through an enhanced leadership framework and successfully implemented program.

2. The greater application uses within the Three Levels of Leadership, the greater the chance of a safer and welcoming school environment for all through an enhanced leadership framework and successfully implemented program.

3. The greater the application uses within School Community, the greater the chance of a safer and welcoming school environment for all through an enhanced leadership framework and successfully implemented program.

4. The higher the degree of non-participation, the less is the chance of program success to ameliorate prejudice behaviors, handle controversial issues and negating situations surrounding diversity in educational leadership.

\section{Action Research Cycle Design}

The researcher's recursive process was used along with the cyclical and spiral experiential learning approach during the action research (Calhoun, 1994; Kemmis, 1988;

Mills, 2011; Stringer, 2007; Wells, 1994) Brown (2009) recognizes the modeled continuum approach through the course of four stages. This action research study incorporated the four stages of Brown's (2009) model into the researcher's cyclical design. These stages are: 
1. Developed leadership team of participants (Bolman \& Deal, 2003; Brown, 2009; DuFour \& DuFour, 2010; DuFour et al., 2006; Kotter, 1996; Muhammad, 2009; Putnam et al., 2009)

2. Evaluation and inquiry (Freeman \& Vasconcelos, 2010; DuFour et al., 2006)

3. Encourage reflective practice (Aguilar, et al., 2004; Compton \& Davis, 2010; Freeman \& Vasconcelos, 2010; Knowles \& Cole, 1996; Schön, 1974).

4. Follow up (Kotter, 1996; McKown, 2005).

In conjunction with the cyclical process used, Brown's model was most helpful to determine the possible benefits of diversity leadership skills training as well as assessed the research problem that needed to be addressed. Participants were able to explore through the modeled exercises as well as discovered ways to help address the research problem with the intent to inform practice through the following action research cycles:

\section{Cycle 1 (May - June 2012)}

Plan. I selected problem of organization's collective inquiry of interest (DuFour et al., 2006). Informed Consent and Consent for Survey were necessary procedures in compliance with IRB standards. Interview protocols and participant pre assessments were administered. Most importantly, I was able to conduct initial information session for introduction to study with developed leadership team of participants (Stage 1). The planned length of module activities was agreed for issuance of eight (8) training clock hours over 8 consecutive weeks during the summer of 2012. 


\section{Cycle 2 \& Cycle 3 (July - September 2012)}

Act \& Collect. Next action taken was to then assess personal experience and observe practice through observational protocols (Creswell, 2007). The commencement of the adapted workshop training model (Brown, 2009) took place. The skills application modules consisted of training modules that spanned over eight consecutive throughout the summer of 2012. Data was collected from the participants and organized for coding scheme (Auerbach \& Silverstein, 2003;

Creswell, 2007). Graph figures were generated to provide an effective visual report from the pre and post assessments (Stage 2).

\section{Cycle 4 (October 2012 - February 2013)}

Reflect, Interpretation, Informed Action. Delivery of change through collective insight and feedback was based upon formative and summative assessment. The objective of the training activities was to maintain an ongoing support group to encourage reflective practice measures (Stage 3) and follow up (Stage 4).

\section{Data Analysis}

Once the surveys, observational and research interview protocol data (Creswell, 2007) were collected, the information was reviewed for a coding (Auerbach \& Silverstein, 2003) to categorize educator comments about diversity and the observed impact on adult faculty/staff from diversity leadership skills training(s). As a result, the compiled coded data was then analyzed in order to make an analysis report (Stringer, 2007) in considering the researcher's recommendation for future research on informed practice. It was further understood by the researcher that this descriptive report did not by any means give any indication on the final results about this subject but rather an analysis on informed practice 
as well as segue for a plan of action towards school implementation. The researcher's recommendations are discussed in Chapter 5, regarding possible action plan strategies in considering the organization's performance improvement efforts and mission revision.

As a result, the compiled coded data was then analyzed in order to make a preliminary report (Stringer, 2007) to later use with the researcher's recommendations on informed practice and future study. Confidentiality was maintained by using Survey Monkey, a commercially available online tool that allowed persons surveyed to anonymously respond via the Internet. No individual results were identifiable unless that person was an educator within the agency who agreed to participate in an interview. That person's identity is known only to the researcher.

Once the surveying was completed, the data collected was compiled together with data collected from those individuals who were interviewed to assess participants' beliefs and impact from skills training received. Assessing the measured impact of diversity leadership training would help in analysis and observation of the applied capacity building skills developed. The initial data received was compiled from the program participants' Likert scale instrumentation (online pre assessments). Again, the methodology used to measure accountability was qualitative based, using the formative/summative assessments and participant evaluation methods: (a) training session observation protocols; (b) pre/post online surveys, measuring participant attitudes and dispositions via Survey Monkey; (c) personal reflection feedback; (d) brief 1:1 stakeholder interviews; (e) quotes from participant learning reflections collected online. Furthermore, the overview of theoretical framework and methodology procedures used for evaluating the efficacy and impact of this 
action research study with focus on the leadership skills application model framework are described further in Chapter 4.

\section{Role of Researcher}

My role as participant observer was to provide the prejudice reduction training as professional development, collect data, analyze, and examine all aspects of the study's assessed data with the intent on identifying whether the variables presented for discussion the study's findings are actual contributing factors surrounding the program's success. Therefore, the role of the researcher is that of recorder, interpreter, and analyzer of the story as told by the interviewees (Marshall \& Rossman, 1999 as referenced in Choi, 2010). The researcher handled leadership and change in this dissertation through the distinct role of participant observer (Creswell, 2007). However, this researcher understood that the role as an action researcher was, according to O'Brien (2001), “to implement the action research method in such a manner as to produce a mutually agreeable outcome for all participants with the process being maintained by them afterwards" (O’Brien, 2001 as referenced in Huntjens, Termeer, Eshuis \& Van Buuren, 2011, p.31).

Moreover, my role of researcher as a change agent was one who had the capability to facilitate and coordinate a change effort or initiative (Wells, 1994; O’Brien, 2001; Huntjens et al., 2011); whereby, according to O'Brien (2001), adopting various roles during the cyclic process to include: catalyzer, teacher, listener, synthesizer, designer, observer and reporter. With this being said, Carnall (2008), Dawson (2010), Stephen (2010) \& Tidd (2010), all define change agents as in my particular role, likewise as playing three distinct roles: consultant, trainer, and researcher. The school leader functions in these capacities as well. 
Lastly, the primary role of the researcher was to support school leaders to the point where they can take responsibility for the change process (Fullen, 2001; Kotter, 1996; Muhammad, 2009; O’Brien, 2001) into implementation. O'Brien (2001) explains further, concluding that this phase is reached when school leaders understand the methods and are able to carry on when the initiating researcher leaves. Chapter 5 will include discussion on the study's summary of findings and recommendations for further directed research as well as describe possible next steps towards a school plan for implementation.

\section{Ethical Practices in My Action Research}

Overall, the analytical methods were mostly descriptive in design (Rubin \& Rubin, 2005). The study's volunteer participants had come from a diverse cross section of the agency's locations, including teachers, central administration, site administrators, and a volunteer Board member. The participants' identities were secured through the use of coding (Auerbach \& Silverstein, 2003). Additionally, reliability and validity in this research design was improved by eliminating any subjectivity or potential biasing factors. The researcher conducted an information session meeting held in collaboration with Central Office administration (June 2012). At this time, this session meeting also served as an orientation for those participants recruited for introduction to study. Recruited participants were even more motivated to receive the eight (8) contact hours of professional development as an incentive for their volunteer time as well as for timely response of online surveying and follow up. Additionally, this effort was intended for practice during the researcher's interviews and observations, assuring that the data collected were dependent on controlled factors without any negating distracters. 
Lastly, all information that could have identified the school or representative(s) was also coded and kept securely stored and locked in a cabinet. Only the researcher had access. Similarly, the participants' confidentiality was secured by using online survey tool and pseudonyms for participants at the conclusion of the study's findings' written analysis. At the end of the study, all information that identified the school or representative(s) was shredded in accordance to IRB regulations. Only demographic information on the school was matched with results.

\section{Discussion on Paradigm Design}

Kotter (1996) \& Fullan (2001) discuss creating major change through effective coalition building of relationships. In coalition building one finds the common elements of agreement among diverse parties in order to accomplish together what each party cannot accomplish alone (Brown, 2009). Additionally, coalition builders attempt to understand the issues of all parties concerned about an issue and to create, where it is feasible, a climate of cooperative cohesiveness. The formation of school improvement planning teams, who are responsible for establishing priorities to effect real change, is significant in the strategic planning process of this study in establishing priorities for improvement through a series of activities. Kotter (1996) identifies these series of activities as "associated with a multistep process that creates power and motivations sufficient to overwhelm all the sources of inertia" and that "this process is never employed effectively unless it is driven by high quality leadership, not just excellent management” (p.19). By modeling Brown’s (2009) workshop modules, the researcher was able to incite shared learning and coalition building (Kotter, 1996). Therefore, it is vital that the participants were all representative of the community. It is important that all team players understand that change is a gradual 
progression of time as they enter into the school improvement planning process. The participants were told this and understood they were embarking in a change process that would take time to accomplish. All the participants were invited to become part of the planning team, and therefore became familiar with the new vision and strategies for the school and its community.

Furthermore, the role of the researcher's methodological focus was to establish evidence of change (Kotter, 1996) for targeted success at reducing prejudice behavior and conduct in the child care agency. When evaluation in conjunction with triangulation methods are used to substantiate such evidence of change, priority, implementation, and integration must be considered (Creswell \& Plano-Clark, 2011; Golafshani, 2003; McKown, 2005; Mills, 2003 \& 2011). Hence, priority is exercised in dedicating a broader emphasis on the study, while implementation is used to determine if the information gathered comes in sequence or chronological stages, and integration presents a phase where merging or connecting the data occurs (Creswell \& Plano-Clark, 2011). Creswell \& PlanoClark (2011) point out that evaluating the conditions and data used in the study provide an understanding of the literature and a measure of frequency in relationship to various variables used in the research and analysis process.

Reflectively, this research study objective and intention was to help participants understand what factors promote changes in reducing prejudice behaviors and or practice (Bolman \& Deal, 2003; Brown, 2009; Clark, 2004; DuFour et al., 2006; Freeman \& Vasconcelos, 2010; Haberman, 1994; Kotter, 1996; McKown, 2005; Purkey, 1992). Again, the compiled information was used in analysis as suggestive data in determining its findings for future research on informed practice so that school practitioners may apply the 
leadership training skills in building a caring school environment and an enhanced leadership framework.

What the researcher did to get the participants commitment and engaged participation was through modeling a new way of training unlike past professional development workshops. By the end of the eight weeks, participants were able to identify and address their own internalized thoughts and behaviors from the module activities and exercises demonstrated. In addition, eight clock hours of professional development training was an added incentive for the participants.

Fundamentally, what the researcher intended to theoretically propose or suggest through inform practice was that the higher the degree of participation; the higher was the chance of program success. These personal theories would perhaps then help to inform on future practices. Therefore, creating major change through effective coalition building of relationships helped to ameliorate prejudice behaviors, handle controversial issues and negating situations surrounding diversity in educational leadership. Finally, the researcher believed there is a window of opportunity based on the receptiveness to what the school learning community can offer through such practiced prejudice reduction models as the ones used in this study. This social justice issue research study was designed as reflected to assist in the support for implementation that urban education programs must eventually experience in the school improvement planning process. 


\section{Chapter 4}

\section{Findings}

The purpose of this action research study was to provide professional development training as part of a continuum change initiative, which included addressing prejudice reduction within the professional development initiatives. Data was also analyzed as part of the change initiative to assess and examine the methods used by individuals and groups (Freeman, 2010; Freeman \& Vasconcelos, 2010; McKown, 2005) to attend to social justice issues involving diversity in educational leadership. In addition, the researcher's primary goal and purpose was to make an attempt in assessing this project's impact as well hope for its success in the continuum for the efficacy of its diversity awareness training and to model and encourage continued support within the organizational framework. Most importantly, the data collected served to inform action on participant's diversity leadership training effectiveness, collective insight, and inform action concerning prejudice behavior amongst educators, and professional development for the purpose of prejudice reduction.

This chapter contains the study findings, interpretation of the findings, analysis and concludes with a discussion on data analysis of the implementation of professional

development. The researcher used the paradigm perspective (Creswell \& Tashakkori, 2007) in the form of action research. As the principal investigator, I used action research as a model form of collective inquiry, not as a method (Creswell \& Tashakkori, 2007). This modeled perspective or viewpoint comes from both qualitative and quantitative methods (Mills, 2011); whereby, the researcher used a selected methodological approach and means of inquiry for data collection. Therefore, my research study was drawn mostly from qualitative embedded methods to include interviewing, reflective journaling, observation 
protocol data, and then drawn upon some quantitative data through survey to measure the efficacy and quality of a prejudice reduction professional development curriculum. Results generated from the collected and coded data were analyzed, which entailed reviewing the documentation gathered from observation and interview protocols as well as retrieving participant survey responses and reflections. The extent to which the results meet the goals and purpose of study will be discovered by the conclusion of this chapter.

The researcher acknowledges that the overall action research approach to organization and coding data is expected to allow themes to emerge from the data and documentation obtained; thus, transforming the delivery of change through collective insight and informed action (Freeman, 2010; Freeman \& Vasconcelos, 2010). The dispositions and attitudes articulated are not necessarily representative of the entire population from those employed by the educational agency. Due to this limitation, the conclusions drawn from this cyclic process should be considered as indicators of successes and needs for improvement based upon findings reported herein. Statistical significance was not applied as a measure for this study. A pre and post comparison analysis was used. Results found from data obtained are reported in terms of their actual frequencies.

\section{Participant Responses}

The study's first subsidiary question sought to measure the impact of diversity leadership training skills on its participants during the eight week module series training. Mills (2011) explains that Likert attitude scales are useful tools for the action researcher to find out "what an individual believes, perceives, or feels" (Gay, Mills, \& Airasian, 2009 as quoted in Mills, 2011, p.91). Research question 1 stated: 


\section{Analytical research question 1.}

What are the effects of informed practice on organizational attitudes and behavior outcomes of education?

This question was posed to determine the degree of change to which the participants might attribute their first thoughts and impact on dispositions about prejudice behavior. More specifically, the focus was connected largely to categorizing educator comments about their perceptions, opinions, beliefs and attitudes and measured impact on participants from newly informed practice in regards to the efficacy and quality of the professional development training to reduce prejudice during professional practice. The responses from five particular survey questions from the participants' pre assessment (See Appendix D) and the five survey questions from the participants' post assessment (See Appendix E) provided useful information to support this question related to the measured impact of diversity leadership training. Likewise, the shared written responses from the participant learning reflections (See Appendix F) also provided meaningful qualitative data about the study's training impact. In addition to examples collected from observation protocol data (See Appendix I) used with the Prejudice Reduction Workshop Model as well as the coded responses from a Board member, Central Office administrators, and site program administrator interview protocol transcripts (See Appendix G \& Appendix H), the collected data provided qualitative information regarding the impact and significance of diversity leadership and professional development. Survey responses are described in the following sections. 


\section{Survey Response}

Quantitatively, question numbers 1,3, 5,6 and 9 on the participant pre and post assessment provided relevant feedback to address the initial and post inquiry of the professional training's impact. The responses were collected, using a Likert type scale with the following choices: 5=Almost Always, 4=Frequently, 3=Sometimes, 2=Rarely, and $1=$ Never. The purposeful sample of training participants was asked to respond online to the following:

Survey Question \#1. I am at ease with people unlike myself.

Survey Question \#3. I am uncomfortable when I hear a prejudicial slur or joke.

Survey Question \#5. I am inclined to let people know that it bothers me when I hear prejudicial statements.

Survey Question \#6. I recognize my own prejudices.

Survey Question \#9. I trust people will fully accept me as ME!

Illustrated in Figure 4-1, the learning group's pre training assessment responses for Survey Question \#1, "I am at ease with people unlike myself," are shown in Figure 4-1.

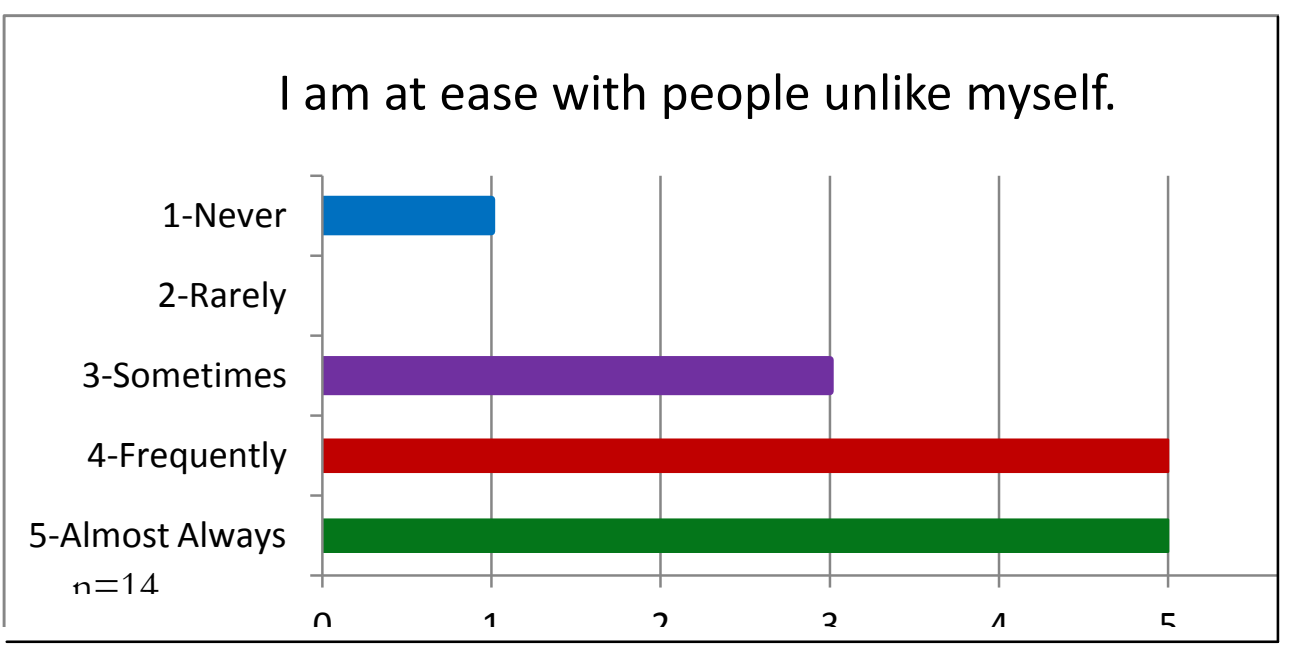

Figure 4-1 Participant Pre Assessment Question 1 
There were a total of 14 participants who completed the survey, and all responded to the survey. Most of their feelings or thinking at that time before training began found that 5 participants (35.7\%) rated almost always, and 5 (35.7\%) rated frequently that they were at ease with people unlike themselves. All other responses included $3(21.4 \%)$ of the staff indicated that they were sometimes at ease with people unlike themselves, and 1 (7.7\%) respondent indicated they never are at ease with people unlike him/herself. The implication was that participants were closed-minded in their tolerance of people unlike them. Further discussion will be included with the post assessment to Question 1.

Illustrated in Figure 4-2, the learning group's post training assessment responses for Survey Question \#1, "I am at ease with people unlike myself," are reflected as shown in Figure 4-2.

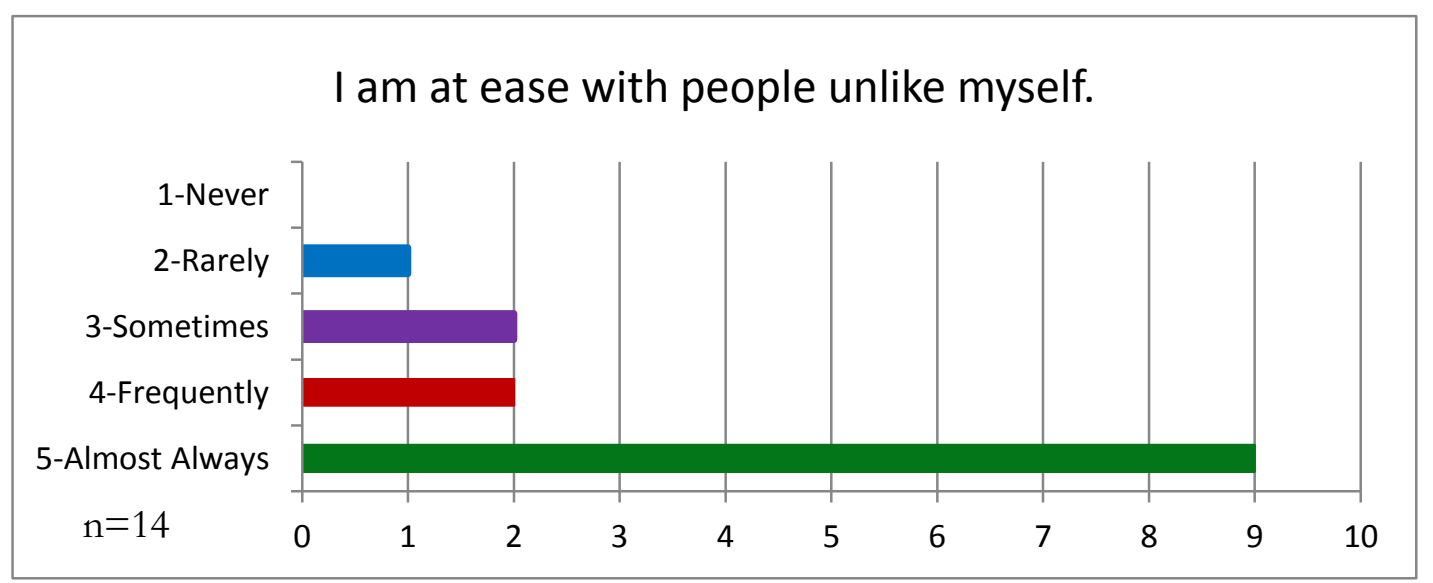

Figure 4-2 Participant Post Assessment Question 1

There were a total of 14 participants who completed the survey, and all responded to the survey. One (7.7\%) respondent showed a disposition change from never to rarely "be at ease with people unlike him/herself." Most of participants' feelings or thinking after 
training found an increase to 9 participants $(64.3 \%)$ that rated almost always. Based on the aforementioned pre assessment taken, there was a $28.6 \%$ increase in change on attitude dispositions concerning ease with people unlike myself. This show more openness towards people unlike them. The increase was significant at $28.6 \%$. All other post assessment responses showed 2 participants $(14.3 \%)$, rating frequently in addition to $2(14.3 \%)$ of the participants indicating that they were sometimes at ease with people unlike themselves.

The first thoughts module exercises were used in which participants discovered and explored in their understanding how stereotypes (records) impact actions and attitudes and how to change individual internalize records (Brown, 2009). From the diversity skills attained, participants were now willing to be aware of and re-examine their dispositions about different groups, individuals, and attitudes that create tension and conflicts in the community.

Illustrated in Figure 4-3, the pre training assessment responses to Survey Question \#3, "I am uncomfortable when I hear a prejudicial slur or joke," are shown below.

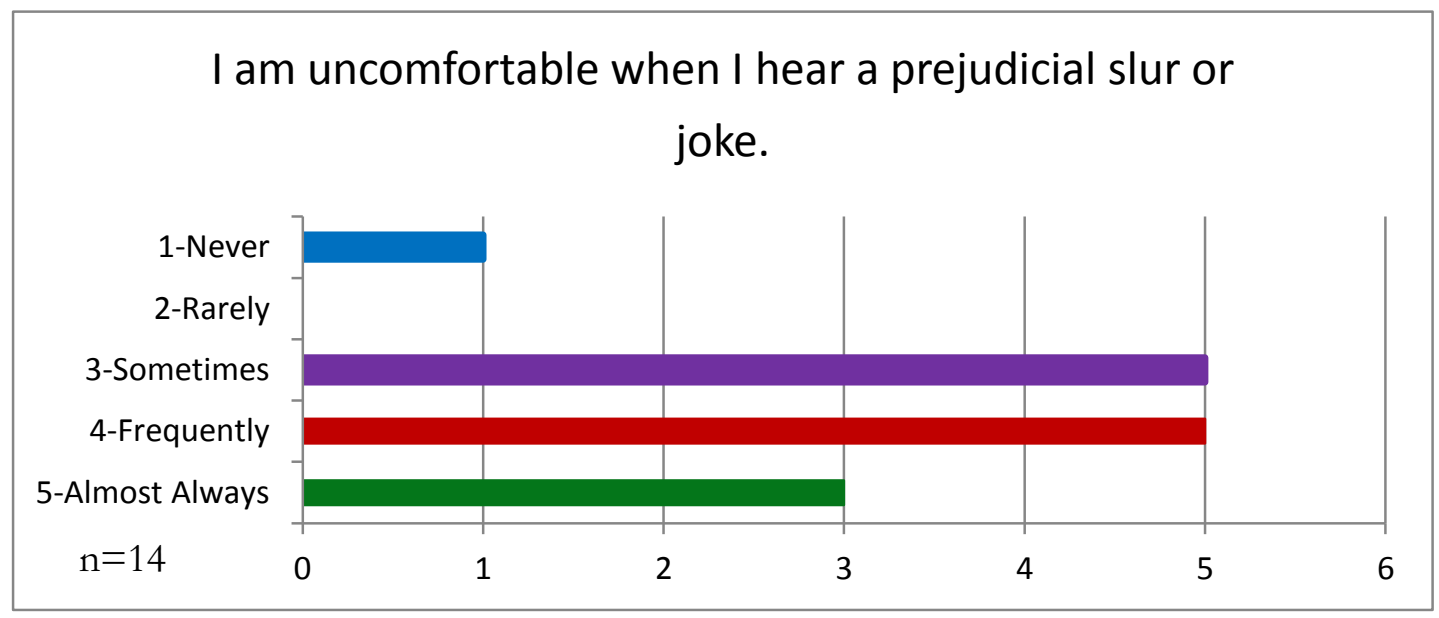

Figure 4-3 Participant Pre Assessment Question 3 
There were a total of 14 out of 14 participants who completed the survey. Most of their feelings or thinking at that time before training began found that 3 participants $(21.4 \%)$ rated almost always, and $5(35.7 \%)$ rated frequently. Also, $3(35.7 \%)$ responded that they were sometimes uncomfortable when they heard a prejudicial slur or joke, and the other $1(7.7 \%)$ respondent indicated they never are uncomfortable when he/she hears a prejudicial slur or joke.

Comparatively as illustrated in Figure 4-4, the learning group's post training assessment responses for Survey Question \#3 “I am uncomfortable when I hear a prejudicial slur or joke," are reflected as shown below.

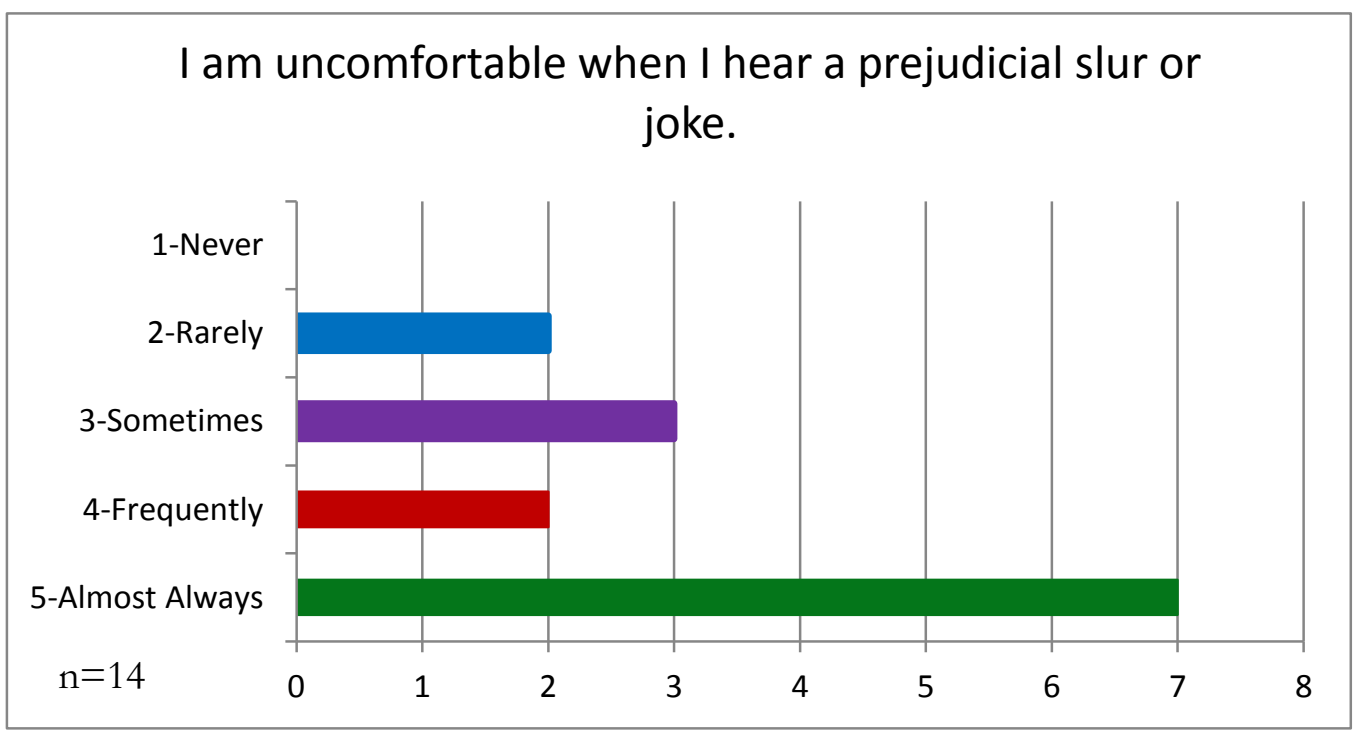

Figure 4-4 Participant Post Assessment Question 3

There were a total of 14 participants who completed the survey, and all responded to the survey. Most of their feelings or thinking after training found an increase to 7 participants (50\%) that rated almost always. Based on the aforementioned pre assessment taken, there was a $28.6 \%$ increase in change on attitude dispositions. All other post 
assessment responses showed $2(14.3 \%)$, rating frequently in addition to $3(21.4 \%)$ of the participants indicating that they were sometimes uncomfortable when they heard a prejudicial slur or joke. Finally, $2(14.3 \%)$ respondents indicated that they are rarely uncomfortable when they heard a prejudicial slur or joke.

Through the training module's caucus group exercises, participants learned how groups have experienced mistreatment and pride. From the diversity skills attained, participants were engaged in learning how other groups experienced prejudice in the community through shared story. In addition, role play skits were observed engaging in modeling how they gained new insight on awareness and information on how different groups experience mistreatment. Participants learned how to be better allies to groups outside of their own.

Moreover, as illustrated in Figure 4-5, the pre training assessment responses to Survey Question \#5, "I am inclined to let people know that it bothers me when I hear prejudicial statements," are shown in Figure 4-5.

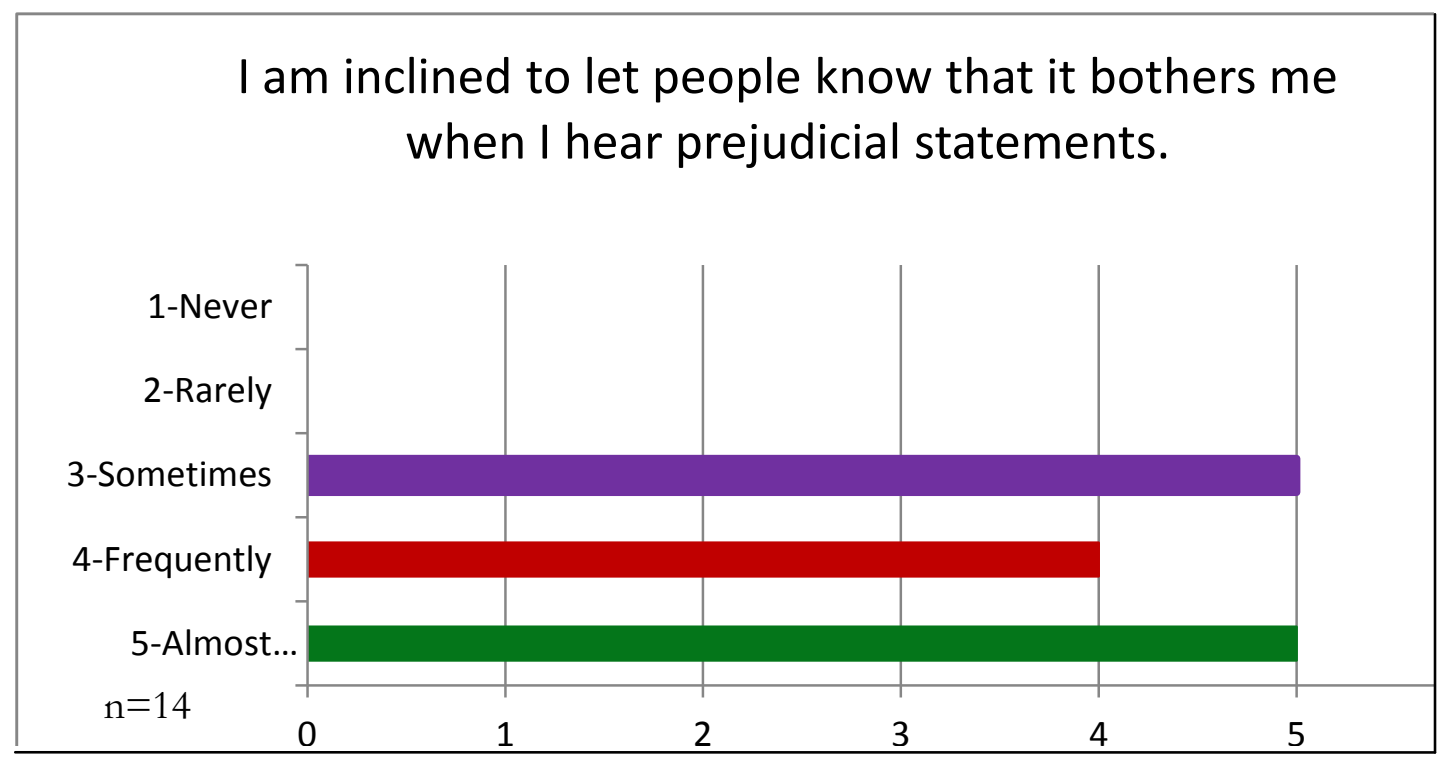

Figure 4-5 Participant Pre Assessment Question 5 
There were a total of 14 participants who completed the survey. All 14 participants responded to the survey. Most of their feelings or thinking at that time before training began found that 5 participants (35.7\%) rated almost always, and 4 (28.6\%) rated frequently. Also, the other $5(35.7 \%)$ responded that they were sometimes inclined to let people know that it bothers them when they heard prejudicial statements.

In contrast as illustrated in Figure 4-6, the learning group's post training assessment responses for Survey Question \#5 "I am inclined to let people know that it bothers me when I hear prejudicial statements," are reflected as shown in Figure 4-6.

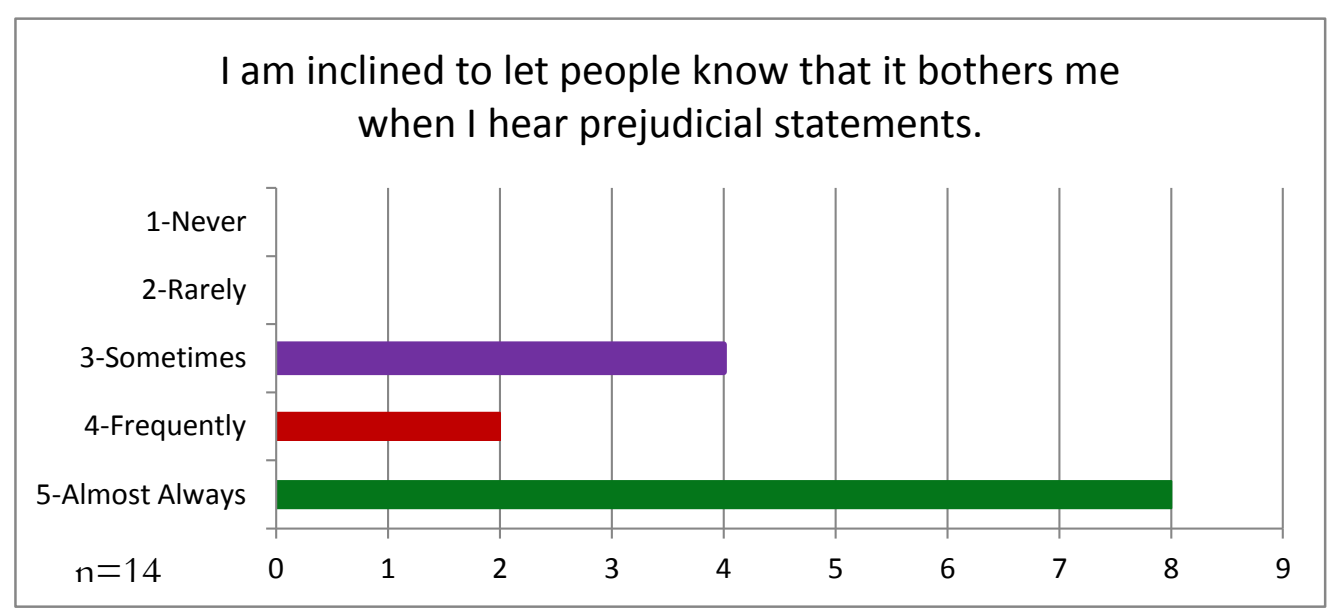

Figure 4-6 Participant Post Assessment Question 5

There were a total of 14 participants who completed the survey, and all responded to the survey. Most of their feelings or thinking after training found an increase to 8 participants (57.1\%) that rated almost always. Based on the aforementioned pre assessment taken, there was a $21.4 \%$ increase in change on attitude dispositions. The implication was that participants were now able to know how to think through conflict and approach situations carefully, especially when hearing prejudicial statements. All other post 
assessment responses showed 2 participants (14.3\%), rating frequently in addition to 4 $(28.6 \%)$ of the participants indicating that they were sometimes inclined to let people know that it bothers them when they heard prejudicial statements.

The speak outs module activity helped participants gain understanding how different types of discrimination effect individuals. This activity was demonstrated and modeled in their learning the power of the personal story and how it can effect change in discriminatory behaviors. In relation to school application, educators were encouraged to remember the painful effect of the stories of discrimination and increase one's commitment to fighting the many "isms" that exist in the community (Brown, 2009). Module workshop activities afforded individuals the opportunity to speak out about their personal experiences involving discrimination and prejudice.

Illustrated in Figure 4-7, pre training assessment responses to Survey Question \#6, "I recognize my own prejudices," are shown in Figure 4-7.

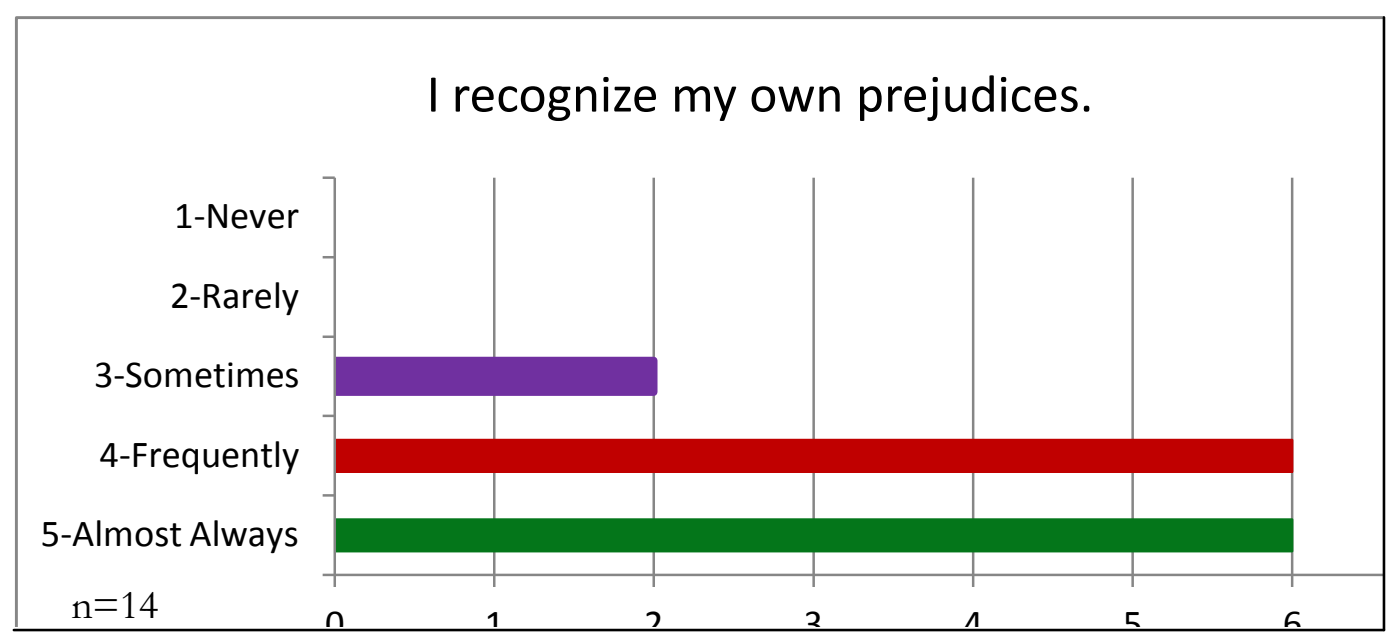

Figure 4-7 Participant Pre Assessment Question 6 
There were a total of 14 participants who completed the survey, and all responded to the survey. Most of their feelings or thinking at that time before training began found that 6 participants (42.9\%) rated almost always, as well as $6(42.9 \%)$ who rated frequently. The other $2(14.3 \%)$ responded that they sometimes recognized their own prejudices.

Significantly as illustrated in Figure 4-8, post training assessment responses for Survey Question \#6 “I recognize my own prejudices," are reflected as shown in Figure 4-8.

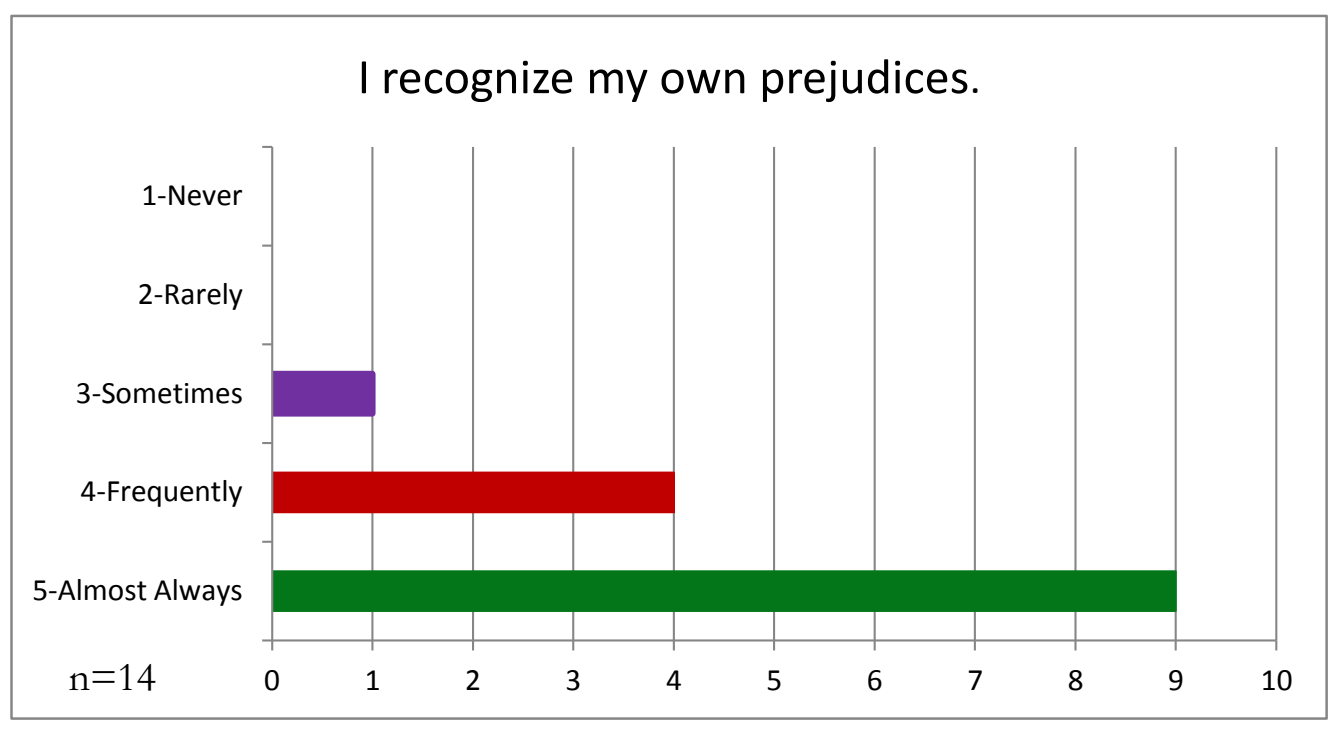

Figure 4-8 Participant Post Assessment Question 6

There were a total of 14 participants who completed the survey, and all responded to the survey. Most of their feelings or thinking after training found an increase to 9 participants (64.3\%) that rated almost always. Based on the aforementioned pre assessment taken, there was a $21.4 \%$ increase in change on attitude dispositions in recognizing one's own prejudices. All other post assessment responses showed 4 participants (28.6\%), rating frequently. The other 1 (28.6\%) respondent indicated that he/she sometimes recognized one's own prejudices. 
Through the learning reflections and volunteered shared stories, participants were able to discover and explore via self examining techniques to find out their own internal records (stereotypes) that have impacted their own behavior. Role Playing and Skits modeled exercises engaged participants to reflect on possible ways to show their commitment to understanding and supporting others' celebrated differences within the learning environment as well as in the communities they resided. For example, the Caucus Groups activity exercises prompted group thinking and strategic thought in considering what possible issues and/or activities could they become involved with at work that would help create a more caring pre-school environment. A participant's learning reflection statement indicated that the "exercises taught me that behaviors can be changed provided that you use the right approach." Another participant's learning reflection statement provided feedback saying that "[t]he most useful part of this workshop was the communication skills needed as well as learning how to express concern.” Furthermore, another participant's learning reflection stated, "I learned what bothers others about their caucus groups.”

Finally, as illustrated in Figure 4-9, the pre training assessment responses from Survey Question \#9, “I trust people will fully accept me as ME!" are shown: 


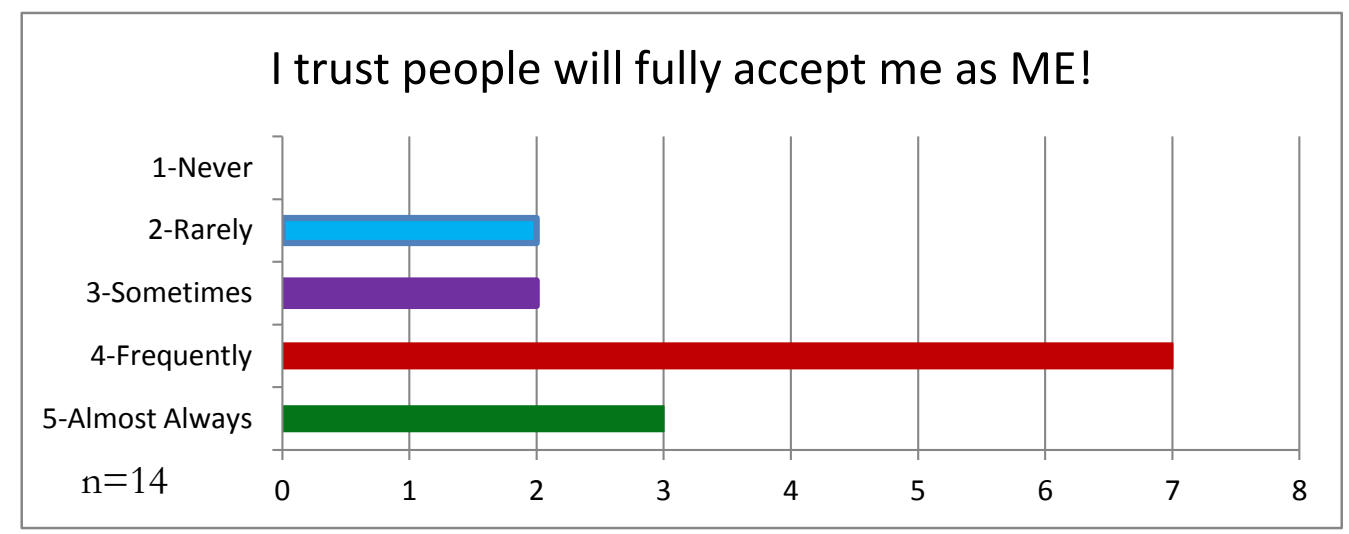

Figure 4-9 Participant Pre Assessment Question 9

There were a total of 14 participants who completed the survey, and all responded to the survey. Most of their feelings or thinking at that time before training began found that only 3 participants (21.4\%) rated almost always; however, 7 (50\%) rated frequently. All other responses showed $2(14.3 \%)$ indicating that they sometimes trust people will fully accept me as ME! Along with 2 (14.3\%) responded rarely.

Comparatively as illustrated in Figure 4-10, post training assessment responses for Survey Question \#9 “I trust people will fully accept me as ME!" are reflected as shown in Figure 4-10.

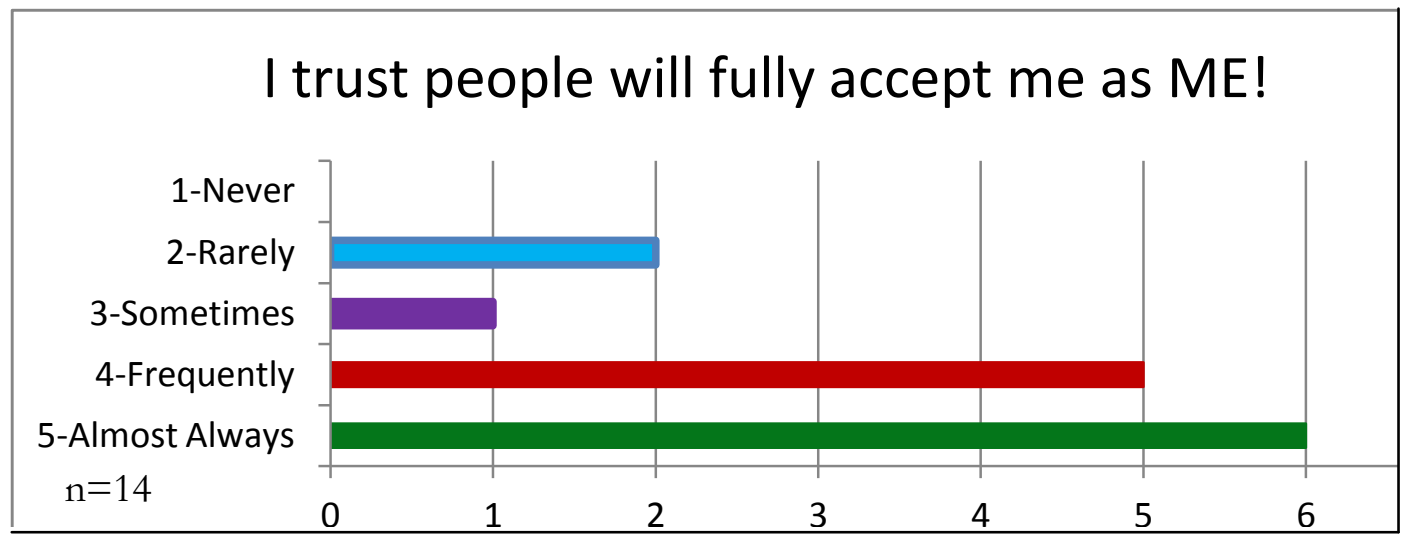

Figure 4-10 Participant Post Assessment Question 9 
There were a total of 14 participants who completed the survey, and all responded to the survey. Most of their feelings or thinking after training found an increase to 6 participants (42.9\%) that rated almost always. Based on the aforementioned pre assessment taken, there was a $21.5 \%$ increase in change on attitude dispositions surrounding self and other acceptance of "me as ME." All other post assessment responses showed 5 participants (35.7\%), rating frequently. The other $1(7.1 \%)$ respondent indicated that he/she sometimes trust people will fully accept me as ME! While 2 (14.3\%) responded rarely.

The pride and capacity building module activities further engaged participants in the delivery of change to express pride in the groups they belong to while supporting others in and out of their group to do the same. The collective insight obtained was observed through group participant discussions in their understanding differences between pride and chauvinism. A participant's learning reflection read, "I learned that if you talk with people, and listen to them, you could perhaps understand more about them and their lifestyles." Another participants reflective feedback read, "Listen more to people, and to appreciate the differences.”

The five pre/post assessment survey questions that were just reported (question numbers 1, 3, 5, 6 and 9) were the most likely to yield results related to the impact of diversity leadership professional development training skills on participants' awareness, beliefs, dispositions and attitudes from newly informed practice. The fact that most of the responses for the five survey questions fell into either the "almost always" or "frequently" categories comparatively suggested that the diversity leadership training workshops did have a significant paradigm shift of thinking and influence on the participants and prejudice reduction and open mindedness to self and others did take place. Feedback from a 
participant's reflection read, "Be aware of others cultural backgrounds." Another

participant reflective feedback read, "Create respectful standards for my classroom environment."

\section{Participant Learning Reflections \& Feedback}

The study's second subsidiary question sought to find out whether or not effective skills application resulted in evidence of change in attitude and behavior outcomes through a pedagogical means by “learning new ways of perceiving people's roles and locations in the perpetuation and resistance of oppressive structures" (Freeman \& Vasconcelos, 2010, p. 8). Research question 2 stated:

\section{Analytical research question 2.}

What factors promote changes in reduction practice of prejudice behavior in schools? What factors impede changes in reduction practice of prejudice behavior in schools?

In order to support the results of the quantitative data from survey responses, qualitative data was also collected from actual shared learning reflections submitted voluntarily by participants (See Appendix F). Additionally, participants were asked to rate their training experience for overall significance and importance of this diversity leadership skills training, using Likert type scale with the following choices: 5=Essential, 4=Very Important, 3=Somewhat Important, 2=Neither Important or Not Important, 1=Not Important. The participants were asked to reflect on the following: "What I learned is...The most useful part of this skills training experience was...”

The participant's reflections presented were taken from a secured data source created online by the researcher, using Survey Monkey, to demonstrate evidence of 
participant views, change of dispositions, and the professional development training evaluation. Learning reflections presented are the actual written responses collected without regard to participants' grammar and or syntax.

\section{Learning Reflection \#1}

"That someone is finally seeking the data. The most useful part of this skills training experience was the information. The delivery of training, specifically on informed practice was engaging with less reading. I have and always will be aware of others cultural backgrounds [and] will continue to practice diversity skills."

My rating for overall significance and importance of this diversity leadership skills training (5-Essential, 4-Very Important, 3-Somewhat Important, 2-Neither Important or Not Important, 1-Not Important): $\underline{\mathbf{5}}$

\section{Learning Reflection \#2}

"To respect the differences in people. The most useful part of this skills training experience was a reminder of professionalism. The delivery of training was on target —no suggestions for improvement. What I learned about myself is, I need to be aware of confidentiality. What I learned about others is that some people are prejudice without even realizing it for themselves. Also, I learned how to better think through situations carefully through the role playing and skits. People are all different and we need to respect those differences. Therefore, I will respect all and do things in a professional manner. I will create respectful standards for my classroom environment." 
My rating for overall significance and importance of this diversity leadership skills training (5-Essential, 4-Very Important, 3-Somewhat Important, 2-Neither Important or Not Important, 1-Not Important): $\underline{\mathbf{5}}$

\section{Learning Reflection \#3}

"Someone actually cares enough to put forth a meaningful study to address the lack of diversity as it relates to educators. The most useful part of this skills training experience was raising awareness for the need for diversity when educating our children. Students need to be exposed to competent educators from all walks of life. Delivery of training was on pointe. I have no suggestions at this time. I still have hope for change. In reality, there are a number of administrators who won't acknowledge the need for diversity training. The exercises taught me that behaviors can be changed provided that you use the right approach. The provided trainer's resource information was helpful. In particular, the statistical analysis was helpful in justifying the need for diversity training. I work with students with disabilities; I will continue to advise them of the importance of not teasing or bulling other students who appear to be social outcast. For the most part the study validates my views on the need for diversity training. I appreciate the opportunity as a participant."

My rating for overall significance and importance of this diversity leadership skills training (5-Essential, 4-Very Important, 3-Somewhat Important, 2-Neither Important nor Not Important, 1-Not Important): $\underline{\mathbf{5}}$ 


\section{Learning Reflection \#4}

"I learned to respect everybody, and Diversity is important to make a contribution to the world. The most useful part of this skills training experience was learning that everybody is unique, and that there is beauty in diversity. Every voice should be heard. More people should receive the training in hospitals, businesses, all kinds of companies, and all grade levels in schools (students). Training was insightful. I learned that I was prejudice. I was closed-minded, and needed to be more tolerant of people that are not like me. Also, other people were prejudice too. Some people were not as open to accept people who did not look like them, think like them, or act like them. I learned that if you talk with people, and listen to them, you could perhaps understand more about them and their lifestyles. Lastly, the trainer's listening skills were meaningful to me. The use of her probing skills did not offend people. Overall, her openness to receive our comments and feedback made the training very engaging. Moving forward, I will learn to listen more to people, and to appreciate the differences. I will be more inviting of different people in my positive efforts to create a welcoming and safe environment for all groups in my school program."

My rating for overall significance and importance of this diversity leadership skills training (5-Essential, 4-Very Important, 3-Somewhat Important, 2-Neither Important nor Not Important, 1-Not Important): $\underline{\mathbf{5}}$ 


\section{Learning Reflection \#5}

"Better understanding after workshop was valuing diversity and the importance of differences and not leaving any group out. Expressing concern was most useful. As far as new insights, I learned about awareness and understanding of other people differences and how people can come together for a main goal. The training leader had us working in groups and expressing ourselves with people different from each other. I would use activity in classroom with children behavior"

My rating for overall significance and importance of this diversity leadership skills training (5-Essential, 4-Very Important, 3-Somewhat Important, 2-Neither Important or Not Important, 1-Not Important): $\underline{4}$

\section{Learning Reflection \#6}

"The importance of learning about different age groups. I learned a new word: ageism! The workshop activities were Excellent. I learned that I am more than just a teacher assistant. I am an Early Childhood Educational leader. I also learned how others feel about different age groups, how to handle conflict and what can I do about it as a teacher. The speak out activity was helpful in learning how to be outspoken. The experience was powerful. I will use skills to do a more better job than ever to be the best teacher ever."

My rating for overall significance and importance of this diversity leadership skills training (5-Essential, 4-Very Important, 3-Somewhat Important, 2-Neither Important or Not Important, 1-Not Important): $\underline{4}$ 


\section{Learning Reflection \#7}

"What I learned is how to deal with diverse groups more effectively. I will be more open minded. The most useful part of the workshop activities was the discussions and advise. Role play and skits activities were helpful in learning that I belong to various caucus groups and what bothers others about their caucus groups. I learned how to be a better leader and how to deal with diversity issues. I feel more confident in being a leader personally and at work."

My rating for overall significance and importance of this diversity leadership skills training (5-Essential, 4-Very Important, 3-Somewhat Important, 2-Neither Important or Not Important, 1-Not Important): $\underline{4}$

\section{Learning Reflection \#8}

"Examining stereotype groups and the importance of being more attentive to all groups is important in respecting diversity. The most useful part of the workshop exercises was learning how I can be an ally to all groups. I learned that I must be acceptable to different diversities. Others have same $1^{\text {st }}$ thought similarities like I did. The trainer was helpful in helping us to understand that we must work together as a teachers for the parents and children. I will use what I have learned with my children and in school at work."

My rating for overall significance and importance of this diversity leadership skills training (5-Essential, 4-Very Important, 3-Somewhat Important, 2-Neither Important or Not Important, 1-Not Important): $\underline{\mathbf{4}}$ 


\section{Learning Reflection \#9}

"I have a better understanding on how I can change the stereotypes (records) I have of others and the impacts of internalized oppression on myself and other groups. I will treat children and adults as both individuals and in group (as needed). I enjoyed doing the pride activities and learning different backgrounds of other cultures of my coworkers; very interesting. I learned the difference between chauvinism and pride. The information I received from this workshop training was helpful. I will be more aware of children's culture and background."

My rating for overall significance and importance of this diversity leadership skills training (5-Essential, 4-Very Important, 3-Somewhat Important, 2-Neither Important or Not Important, 1-Not Important): $\underline{4}$

\section{Learning Reflection \#10}

"The workshop training activities and training presentation were excellent. The speak out exercises were insightful in learning more about myself and about others prejudices. The training presenter explained more about how the different types of discrimination affect people, stereotypes, what internalized oppression is, and the difference between chauvinism and pride. I learned that others do their best not to be prejudice without realizing they really are."

My rating for overall significance and importance of this diversity leadership skills training (5-Essential, 4-Very Important, 3-Somewhat Important, 2-Neither Important or Not Important, 1-Not Important): $\underline{4}$ 


\section{Learning Reflection \#11}

"I learned about the various group to which people belong and are involved in that affect their behavior. I will be considerate and aware of children's behaviors in from more points of view. I will be more understanding according to the groups they belong in order to understand and meet their needs better. The training information given was very useful and knowledgeable. What I learned about myself is my behavior is due to the various groups I belong, and others behave due to the various groups they belong. I have learned to be objective and accept people for who they are. The trainer helped us in exposing the things that affect or influence behaviors. I will forgive and forget, not just be fair but move on from being fair to be a great."

My rating for overall significance and importance of this diversity leadership skills training (5-Essential, 4-Very Important, 3-Somewhat Important, 2-Neither Important or Not Important, 1-Not Important): $\underline{4}$

\section{Learning Reflection \#12}

“What I learned is the art of listening to others' stories of discrimination and how they were affected. Learning how to just listen more carefully to other people and what they do differently was helpful in my understanding of the training. The ups and downs activity was useful in understanding different types of ways people may discriminate. The training was helpful to learn how the children look up to you and you must model appropriate behavior working as a teacher. The workshop group activities were effective in the training." 
My rating for overall significance and importance of this diversity leadership skills training (5-Essential, 4-Very Important, 3-Somewhat Important, 2-Neither Important or Not Important, 1-Not Important): $\underline{4}$

\section{Learning Reflection \#13}

"What I learned is how to be proactive, and how I have to learn how to listen more and be aware of others' differences. Listening to others shared stories is important in order to be effective allies to other groups in the workplace. The closing circles at the end of each workshop training were memorable. With everyone standing and holding hands, we were asked by the trainer to say a highlight from the day. This was our time to individually share, and say something that touched us, or share something that we learned. In general, the trainer was inspiring, ending the workshops with something positive and thought provoking with a quote or a songShe actually had everyone SINGING!!! Diversity matters, and the training taught me how to go about to prevent incidents of inappropriate behavior." My rating for overall significance and importance of this diversity leadership skills training (5-Essential, 4-Very Important, 3-Somewhat Important, 2-Neither Important or Not Important, 1-Not Important): $\underline{4}$

\section{Learning Reflection \#14}

"I learned other things about people of other groups, which was helpful to me in learning about other stereotypes. I learned how I can be an ally to other groups whether at work or in my community. Everything was very helpful to me." 
My rating for overall significance and importance of this diversity leadership

skills training (5-Essential, 4-Very Important, 3-Somewhat Important, 2-Neither

Important or Not Important, 1-Not Important): $\underline{4}$

The shared participant learning reflections, in addition to examples collected from observation protocol data (See Appendix I), used with the Prejudice Reduction Workshop Model supported the researcher's formative evaluation results of the pre and post assessment survey questions. Their learning experience was enhanced by the eight week diversity leadership training skills workshops and module activities. Most of the written reflections shared online and those observed shared amongst workshop participants during trainings supported the researcher's contact hypothesis implied on informed practice through peer training and professional development activities. Qualitatively, these measures enhanced the quantitative from the pre and post assessment responses collected. This study took on the understanding that prejudice is learned and "that prejudice and interpersonal function can be changed through education" (Spiotta, 2004, p.15).

Formatively, the researcher was able to affirm the contact hypothesis based on the participant post assessment data findings, individual participant's feedback after each training workshop as well as summative found evident in review of the individual participant reflective submissions. Specifically, a closing circle (Brown, 2009; NCBI, 2010 \& 2011) with participants observed standing and holding hands was part of the capacity building activities to end each day's workshop. The researcher as training facilitator asked each participant, starting with the person on the left, to share their personal goal intended to apply new workshop skill and their highlight from the day. The highlight was defined as 
something that touched them or something that they learned. In addition, participants were asked to say one thing they appreciated (Aguilar, et al., 2004) about the trainer's leadership.

Thusly, the study's purpose for the efficacy and impact of the prejudice reduction professional development training, manifested over the course of the eight weeks. In addition, the study successfully addressed the primary research question: How does diversity leadership training skills on prejudice reduction result in changed beliefs of discriminatory behavior in schools? The individual training workshops' observational protocol coding document is included in this chapter. The actual coding scheme to categorize educator comments about diversity leadership skills and school application will be shown as illustrated in matrix tables at the latter section of this chapter under Observational Protocol Data.

\section{Interview Responses}

This study attempted to survey a valid representation of a purposeful sample of total participants in the program to include a Board member, Central Office administrators and site lead administration respectively. Members of the study group were densely interconnected; whereby, snowball or chain referral sampling techniques was deemed best appropriate for the sample to minimize bias while maintaining privacy and confidentiality (Marshall, 2003). In addition, the beliefs and attitudes expressed are not necessarily representative of the entire population from those employed by the educational agency. Due to this limitation, the conclusions drawn from this cyclic process should be considered as indicators of successes and needs for improvement based on findings.

Each interview subject received communication from the researcher for an informal interview. Neither gender nor race had any measure in this action research study. 
Qualitatively driven data collection and some measure of quantitative analyses of the survey and interview results were performed respectively.

\section{Procedures for Answering Research Questions}

The pre and post assessment surveys were designed to answer Analytical Research Question 1. The participant learning reflections in addition to examples collected from observation protocol data were designed to answer Analytical Research Question 2. The interview protocols were designed to collect data from coding scheme to agency interview protocol transcripts to answer the third subsidiary question. Research question 3 stated:

\section{Analytical research question 3.}

Based on the data collected from the findings, how can the diversity leadership training be strengthened for the organization?

In addition to the triangulated data taken from assessment surveys, observation protocol data, reflective journaling, and participant evaluated training feedback, additional qualitative data came from the organization's program and site administration leadership. The interviews (See Appendix G \& Appendix H) were conducted in the initial cycle of the researcher's action research. Once informed consent and consent for survey were obtained, interviews were completed along with the participant sample's pre assessment surveys. The plan for the eight week training was mapped out in collaboration with Central Office administration during an information session (June 2012). This meeting also served as an orientation for those participants recruited for introduction to study.

\section{Findings Supporting Primary Research Question}

One of the interview questions clearly supported the primary research question, "How does diversity leadership training skills on prejudice reduction result in changed 
beliefs of discriminatory behavior in schools?" The matrix tables (Table 4-1 and Table 4-2) are excerpts from the actual coding scheme to Interview Question 9, "What professional development activities and support will equip staff with the needed knowledge and skills to perform effectively and achieve desired results? Who is responsible for the various actions?" The following excerpts are coded scheme responses from a Board member (B), two administrators $\left(\mathrm{A}^{1}\right.$ and $\left.\mathrm{A}^{2}\right)$, and site supervisor/center director participants $\left(\mathrm{C}^{1}, \mathrm{C}^{2}, \mathrm{C}^{3}\right.$, and $\mathrm{C} 4)$ :

Table 4-1

Diversity. Central Administration

\begin{tabular}{l|l}
\hline $\begin{array}{l}\text { Coding } \\
\text { Category/Label }\end{array}$ & Example from Data Collection/Criteria (Interview Protocol) \\
\hline $\mathrm{Q}_{9}$ & $\begin{array}{l}\text { B -Workshops and hands on training are essential in understanding } \\
\text { the reality of the diverse world we lived in today. The need for } \\
\text { \& Accountability } \\
\text { social skills, which emphasizes equality of results when interacting } \\
\text { with a diversity of people. } \\
\mathrm{A}^{1}-\text { Administrators, teachers, student, parents and non- } \\
\text { instructional need to be provided with the proper professional } \\
\text { development programs and be instructed by experts in the field. } \\
\mathrm{A}^{2}-\text { Allocating funds to attend conferences, and return to share } \\
\text { learned experiences in our in-services. }\end{array}$ \\
\hline
\end{tabular}


Table 4-2

Diversity. Site Administration

\begin{tabular}{l|l}
\hline $\begin{array}{l}\text { Coding } \\
\text { Category/Label }\end{array}$ & Example from Data Collection/Criteria (Interview Protocol) \\
\hline Q $_{9}$ & $\mathrm{C}^{1}-$ More access to current reading materials \\
$\begin{array}{l}\text { Professional Development } \\
\text { (Support) \& }\end{array}$ & $\mathrm{C}^{2}-$ Monthly meetings \\
Accountability & $\mathrm{C}^{3}$-Participation in more training with mentoring support such as \\
& $\begin{array}{l}\text { the Thomas Edison State College English Language Learners } \\
\text { Summer Institute. } \\
\end{array}$ \\
& $\begin{array}{l}4 \\
\text { should facilitate developmental activities. }\end{array}$ \\
\hline
\end{tabular}

\section{Observational Protocol Data}

Based upon the conceptual framework of Brown (2009) and Clark's (2004) adapted performance improvement conceptual mapping approach (Chapter 2, Figure 2-4), I incorporated the workshop model's skills application training in considering my constructed observational protocol (Creswell, 2007). As a result, the following matrix tables were created by the researcher to contain the actual coding scheme and categorized educator comments about diversity leadership skills and school application from the eight week professional development training. The categories were based on examples collected from observation protocol data used with the Prejudice Reduction Workshop Model's eight week module activities. Some of the coding references for each workshop are expressed in a word or short phrase while other coding references are expressed in one or more phrases: 
Table 4-3

Diversity Leadership Skills.

\begin{tabular}{l|l}
\hline Coding Category/Label & $\begin{array}{l}\text { Example from Data Collection/Criteria (Observation } \\
\text { Protocol) }\end{array}$ \\
\hline Workshop \#1: & $\begin{array}{r}\text { Refers to knowing and practicing: Code Awareness } \\
\text { - Levels of Leadership }\end{array}$ \\
& $\begin{array}{l}\text { - Self Awareness - examine self } \\
\text { - Sther Awareness - being an ally to others }\end{array}$ \\
\hline
\end{tabular}

Table 4-4

School Application.

\begin{tabular}{l|l}
\hline Coding Category/Label & $\begin{array}{l}\text { Example from Data Collection/Criteria (Observation } \\
\text { Protocol) }\end{array}$ \\
\hline Workshop \#1: & $\begin{array}{l}\text { Refers to recognizing that in whatever roles we have within } \\
\text { the school, we can take different levels of leadership to } \\
\text { enhance the school environment for ourselves and others. } \\
\text { Code Organizational Culture and EQ (Emotional } \\
\text { Intelligence) }\end{array}$ \\
$\begin{array}{l}\text { Peers (including student, } \\
\text { faculty, and staff leaders) }\end{array}$ & \\
\hline
\end{tabular}

Workshop \#1. Three levels of leadership/school community as peers (including student, faculty, and staff leaders).

This is what I asked (NCBI Trainer's Notes as referenced in Brown, 2009, p.5):

Each person stood and introduced themselves (name and site location). Share... "something they may want others to know about them that they might otherwise not know" or "the work that has their heart.” 
This is why I asked it: to get everyone to acknowledge everyone in their roles at the various levels of the leadership within the organization.

This is what I observed and heard: everyone stood for their introduction, speaking loud enough for all to hear their name and site location. The theme around the room was centered on "Children First" as childhood educators and administrators for the betterment of their professional development.

This is the impact: celebrated appreciation and recognition of each other's roles.

Table 4-5

Diversity Leadership Skills.

\begin{tabular}{l|l}
\hline $\begin{array}{l}\text { Coding } \\
\text { Category/Label }\end{array}$ & Example from Data Collection/Criteria (Observation Protocol) \\
\hline Workshop \#2: & $\begin{array}{l}\text { Refers to respecting and welcoming diversity in all forms - "Every } \\
\text { Group Counts." } \\
\text { Environmental } \\
\text { Scanning }\end{array}$ \\
$\begin{array}{l}\text { Code Asking: "Did we leave anybody out?"; Code Behavior and } \\
\text { Conduct }\end{array}$ \\
\hline
\end{tabular}

Table 4-6

School Application.

\begin{tabular}{l|l}
\hline $\begin{array}{l}\text { Coding } \\
\text { Category/Label }\end{array}$ & Example from Data Collection/Criteria (Observation Protocol) \\
\hline Workshop \#2: & $\begin{array}{l}\text { Educators learn how to notice which school groups get respect and } \\
\text { which groups are excluded and reaching out to them. } \\
\text { Code Abilities (Human Capacities) }\end{array}$ \\
\hline
\end{tabular}




\section{Workshop \#2. Environmental scanning: Ups/Downs activity.}

This is what I asked (NCBI Trainer's Notes as referenced in Brown, 2009, p.7): When you are a member of a group (i.e., family order, place of birth, ethnic/cultural groups, national heritage, religion, class/economic background growing up, age, gender, visible or invisible disability, vegetarians, friends/relatives who identify as gay, lesbian, bisexual, transgender, etc.), please stand. For anyone who is physically challenged and cannot stand, please put up your hand to let us know that you are part of that group. When groups other than your own stand, those who are seated should applaud, wave, cheer them on in a welcoming manner. Which group did I leave out? For those who do want to identify as being GLBT, I would like to give you the chance to stand and to proudly share that. Even if you choose not to identify here, you can still be completely proud. For those who identify as GLBT in this room or any other group and decided not to be out, let's applaud. Any broad category of private identities that anyone wished to name the group (i.e. single parents, divorced, adult children of alcoholics) were invited to stand and be applauded.

This is why I asked it: for all to learn about the many similarities and differences among participants.

This is what I observed and heard: Participants looking around as they each stood for the group(s) they identified with along with others applauding while seated anticipating their turn to jump up for their identified group. Group's choice was then offered to turn to the person seated next to them, and come up with one question they would like to know about each other that was phrased in a manner where the audience could answer with a total physical response of standing up and sitting down. If they agreed, they stood. If they disagreed they sat (up/down question). 
This is the impact: ice breaker; warmed up participants to comfortably interact with each other; reinforced the topic of the training's purpose. Participants learned something about each other.

Table 4-7

Diversity Leadership Skills.

\begin{tabular}{l|l}
\hline $\begin{array}{l}\text { Coding } \\
\text { Category/Label }\end{array}$ & Example from Data Collection/Criteria (Observation Protocol) \\
\hline Workshop \#3: & $\begin{array}{l}\text { Refers to understanding how stereotypes impact our actions and } \\
\text { attitudes and how we can change them. }\end{array}$ \\
$\begin{array}{l}\text { Stereotyping: } 1^{\text {st }} \\
\text { Thoughts }\end{array}$ & Code Values and Beliefs \\
\hline
\end{tabular}

Table 4-8

School Application.

\begin{tabular}{l|l}
\hline $\begin{array}{l}\text { Coding } \\
\text { Category/Label }\end{array}$ & Example from Data Collection/Criteria (Observation Protocol) \\
\hline Workshop \#3: & $\begin{array}{l}\text { Educators learn through these demonstrated exercises how to } \\
\text { effectively examine the stereotypes within the school that create } \\
\text { tension and conflict. } \\
\text { First Thoughts } \\
\text { Code Learning by Doing (Explicit knowledge into Tacit } \\
\text { knowledge); Espoused (Disposition) }\end{array}$ \\
\hline
\end{tabular}

Workshop \#3. Stereotyping: $1^{\text {st }}$ thoughts/First thoughts exercise.

This is what I asked (NCBI Trainer's Notes as referenced in Brown, 2009, p.13):

Asked participants to pair up and select a group to which neither belongs. Select a group to 
which someone else in the room belongs. Choose a term that may not be the safest to work with since this workshop is about taking risks. Prior to asking group to share their first thought with each other before the whole group, trainer had participants open their arms wide and turned towards their partner to say, "I love taking risks; I love making mistakes!"...I welcome you to take risks and make mistakes about me and my group(s).” Last question: What was it like to hear those first thoughts about your group?

This is why I asked it: (1) to get prejudicial ideas out into the open; (2) to get rid of them; and, (3) to help participants look at the various stereotypes they have learned and internalized about groups other than their own, forming a literal record. According to Brown (2009), "when our thoughts and experiences refute these records, they still exist and influence our behavior (p.13)." Reminded participants that it is only when they are willing to take risks that their internal records about each other begin to lose their power over them. Brown states, “...for some of us the real risk is letting people get close enough to make mistakes about us (p.14).

This is what I observed and heard: a participant (white female) was trying to censor her words out of respect to those who identified as African American when she was given the word "Camden"; whereas, others took the opportunity as another fun activity to speak and share exactly what they internalized about the terms: Jamaicans, Puerto Ricans, ghetto, people who lived in trailer parks, people on food stamps, etc.

This is the impact: 'ah-ha' moments for some in that fear to speak and share about one's own developed stereotypes about other groups. 
Table 4-9

Diversity Leadership Skills.

\begin{tabular}{l|l}
\hline $\begin{array}{l}\text { Coding } \\
\text { Category/Label }\end{array}$ & Example from Data Collection/Criteria (Observation Protocol) \\
\hline Workshop \#4: & $\begin{array}{l}\text { Refers to learning the negative impact that stereotypes have on our } \\
\text { own and different groups' self image and behavior. } \\
\text { Internalized } \\
\text { Oppression }\end{array}$ \\
\hline
\end{tabular}

Table 4-10

School Application.

\begin{tabular}{l|l}
\hline $\begin{array}{l}\text { Coding } \\
\text { Category/Label }\end{array}$ & Example from Data Collection/Criteria (Observation Protocol) \\
\hline Workshop \#4: & $\begin{array}{l}\text { Refers to learned conflict resolution skills for handling and } \\
\text { resolving tough interpersonal/intergroup conflicts. Code Exploring } \\
\text { ways to "contradict" and to interrupt the internalization of } \\
\text { negative stereotypes on all groups and reduce behaviors towards } \\
\text { them. }\end{array}$ \\
\hline \begin{tabular}{l} 
Process \\
\hline
\end{tabular}
\end{tabular}

Workshop \#4. Internalized oppression/Controversial issues process.

This is what I asked (NCBI Trainer's Notes as referenced in Brown, 2009, p.16): In pairs, pick a group to which you personally belong and point your finger at your partner and say, "What I can't stand or what bugs me the most about you...[for example] is..." Each will have a turn in doing this, picking a group to which you belong and do the same. At once the group returned from the pairs exercise, I first asked how many found this exercise harder than the First Thoughts workshop. Follow up question was asking them why it was harder and or why was it easier. Lastly, participants were asked to share in their 
responses to the group(s) picked and what were some of the things they could not stand about their respective groups to which they personally belong. Concluded exercise with asking participants to discuss what all of the things people cannot stand about their groups have in common.

This is why I asked it: (1) to understand how diversity work goes better when participants learn about the painful internalized feelings within each group; (2) to look at the less than positive feelings participants have about members of their own group. Action of pointing exercise was encouraged to help in the process of getting rid of the negative feelings. To understand how similar participants groups' struggles are. Participants began to engage in capacity building across group lines.

This is what I observed and heard: Participants initially expressed concern of their not being used to pointing fingers at people in particular now circulating among the pairs with each other for this exercise. Noted that some people found it very easy to openly say the things they could not stand about their own group. In addition, an African American male participant expressed how it might not be a good idea to say the things we cannot stand about our own group around 'other' people.

This is the impact: Participants observed and heard how the impact of internalized stereotyping affected others. They gained a better understanding and appreciation of the struggles of each group, reframing dispositions. 
Table 4-11

Diversity Leadership Skills.

\begin{tabular}{l|l}
\hline $\begin{array}{l}\text { Coding } \\
\text { Category/Label }\end{array}$ & Example from Data Collection/Criteria (Observation Protocol) \\
\hline Workshop \#5: & $\begin{array}{l}\text { Refers to encouraging the expression of pride in all groups and } \\
\text { understanding the difference between authentic pride and } \\
\text { chauvinism. Code Motivation \& Capacity Building }\end{array}$ \\
$\begin{array}{l}\text { Building Pride: "It's } \\
\text { Great To Be" }\end{array}$ & \\
\hline
\end{tabular}

Table 4-12

School Application.

\begin{tabular}{l|l}
\hline $\begin{array}{l}\text { Coding } \\
\text { Category/Label }\end{array}$ & Example from Data Collection/Criteria (Observation Protocol) \\
\hline Workshop \#5: & $\begin{array}{l}\text { Creating opportunities for students, staff, and all groups to express } \\
\text { pride and receive recognition. }\end{array}$ \\
$\begin{array}{l}\text { Building Capacity \& } \\
\text { Authentic Cross } \\
\text { Cultural } \\
\text { Relationships }\end{array}$ & Code Motivation and Engagement \\
\hline
\end{tabular}

Workshop \#5. Building pride: “It's Great To Be"/Building capacity \& authentic cross cultural relationships.

This is what I asked (NCBI Trainer's Notes as referenced in Brown, 2009, p.18):

Return to your previous partner from last workshop. Take to the same group to which they

just said, "What I can't stand about you..." and this time say, "What I am most proud

of..." Invited participants to share what they are most proud of and or love about their own groups. Next, asked what groups participants are a member of that they are not yet fully proud of being a member of that group. In addition, I asked, "What were groups that they 
could not easily say it was great to be a member of that group?" Asked participants to share, asking, "What is it great to be?" Researcher, as trainer demonstrated, leaped in the air, saying “It's great to be...!” I encouraged training participants to applaud enthusiastically.

This is why I asked it: (1) to not leave participants having said only the negative things about their own group; (2) to look at any of the places where participants are less than fully proud of their own groups; (3) to reach for a sense of complete pride in the participants; (4) to understand the difference between pride (love) and chauvinism (your group is better than the other to conceal feelings of inferiority); and, (5) to learn that authentic pride welcomes diversity.

This is what I observed and heard: Participant were allowed to call out a sampling of the groups that they are less than fully proud of (i.e. adult children of alcoholics, adult children of abuse, domestic violence, those who were raised with less than enough growing up, being from Camden, N.J., etc.). Researcher as trainer had participants engaged in pairs to choose a group they may felt or people mistakenly think ought to feel ashamed. Each shared in front of the room, rotating pairs. One person would leap into the air, saying, "It's great to be FROM CAMDEN!" The partners then switched modeling the same about their chosen group and then created fun categories...Laughter and applause.

This is the impact: Participants' affirmation and celebrated recognition of the groups to which they personally belong. 
Table 4-13

Diversity Leadership Skills.

\begin{tabular}{l|l}
\hline $\begin{array}{l}\text { Coding } \\
\text { Category/Label }\end{array}$ & Example from Data Collection/Criteria (Observation Protocol) \\
\hline Workshop \#6: & $\begin{array}{l}\text { Educators gain a new perspective on awareness and information } \\
\text { on how different groups experience mistreatment and learning } \\
\text { how to be better allies to them. } \\
\text { Becoming Allies }\end{array}$ \\
\hline
\end{tabular}

Table 4-14

School Application.

\begin{tabular}{l|l}
\hline $\begin{array}{l}\text { Coding } \\
\text { Category/Label }\end{array}$ & Example from Data Collection/Criteria (Observation Protocol) \\
\hline Workshop \#6: & $\begin{array}{l}\text { Identifying school groups where there is mistreatment and } \\
\text { misinformation and correcting it. } \\
\text { Caucus Groups }\end{array}$ \\
\hline
\end{tabular}

Workshop \#6. Becoming allies/Caucus groups.

This is what I asked (NCBI Trainer's Notes as referenced in Brown, 2009, 21): List of the caucus groups that participants would like to present to the workshop. In pairs, asked participants to think of one or two groups that they would want for their own caucus, a group to which they personally belong, either currently or from their past or a group that has experienced some form of discrimination. Think about a group you know that people have less than positive feelings about. Encouraged to choose a group that participants think 
the workshop needs to hear from. Asked about common themes, what touched you, what was new information, etc.

This is why I asked it: (1) to learn about the many different ways people have experienced discrimination and to begin to learn how to stop the prejudicial behavior; (2) to have participants think of one group to which they belong which has experienced some kind of discrimination; (3) to have participants meet in a caucus of several other people from their same group; (4) to give individual caucus reports on the represented groups; and, (5) to listen and inform on caucus groups reports.

This is what I observed and heard: Examples displayed on the flip charts around the room that read: Black/African Heritage; Latinos; Jews; White/European Heritage; Women; Men; Young People; GLBT; Welfare Recipients; Single Mothers, Camden Residents, etc. Each caucus gave an oral presentation to the whole workshop that answered the question displayed on the power point slide: "WHAT DO YOU NEVER AGAIN WANT PEOPLE TO SAY, THINK OR DO TOWARD YOU GROUP?”

This is the impact: renewed awareness in prejudice behaviors.

Table 4-15

Diversity Leadership Skills.

\begin{tabular}{l|l}
\hline $\begin{array}{l}\text { Coding } \\
\text { Category/Label }\end{array}$ & Example from Data Collection/Criteria (Observation Protocol) \\
\hline Workshop \#7: & $\begin{array}{l}\text { Educators exercise listening skills in learning how different types } \\
\text { of discrimination affect individuals and groups. }\end{array}$ \\
$\begin{array}{l}\text { Making } \\
\text { Commitments to }\end{array}$ & $\begin{array}{l}\text { Code Learn the power of the personal story and how it can effect } \\
\text { change. }\end{array}$ \\
\hline
\end{tabular}


Table 4-16

School Application.

\begin{tabular}{l|l}
\hline $\begin{array}{l}\text { Coding } \\
\text { Category/Label }\end{array}$ & Example from Data Collection/Criteria (Observation Protocol) \\
\hline Workshop \#7: & $\begin{array}{l}\text { Refers specifically to capacity building through group work and } \\
\text { determining the Speak Outs that need to be told and heard in order } \\
\text { to make the school environment safe and welcoming for all. } \\
\text { Code Engagement }\end{array}$ \\
\hline
\end{tabular}

Workshop \#7. Making commitments to change/Speak outs.

This is what I asked (NCBI Trainer's Notes as referenced in Brown, 2009, p. 26):

The workshop began with a warm up, asking participants who has a name that people never say correctly and to stand. Afterwards, I asked participant volunteers to come up front of the workshop to share a personal experience of discrimination. Instructions given were to share an incident where you have experienced mistreatment that is painful and one that you would be willing to share with the workshop group.

This is why I asked it: to learn that the sharing of stories, because they are so personal, change people from the heart in hearing actual personal experiences.

This is what I observed and heard: Participants were individually given an opportunity to say his/her name properly. The workshop participants were then instructed to repeat that individual's name. Next, participants shared individual stories of personal discrimination. The workshop tone became intimate and emotional, for example, there was crying on the part of the storyteller. An audience participant spontaneously came up to the front to hug the storyteller, and so forth. Physical displays of compassion shown. 
This is the impact: Participants were emotionally engaged.

Table 4-17

Diversity Leadership Skills.

\begin{tabular}{l|l}
\hline $\begin{array}{l}\text { Coding } \\
\text { Category/Label }\end{array}$ & Example from Data Collection/Criteria (Observation Protocol) \\
\hline Workshop \#8: & $\begin{array}{l}\text { Refers to reflective learning practicing in knowing the 3 Levels of } \\
\text { Leadership as an ally for preventing and interrupting prejudicial } \\
\text { jokes, comments, and slurs. Noticing and supporting both the } \\
\text { perpetrator and victim of prejudicial jokes, comments and slurs. } \\
\text { Code Learning by Doing (Explicit knowledge into Tacit } \\
\text { knowledge) }\end{array}$ \\
\hline
\end{tabular}

Table 4-18

School Application.

Coding Category/Label

Workshop \#8:

Role Playing and Skits
Example from Data Collection/Criteria (Observation Protocol)

Refers to demonstration and application in identifying the most common situations in school where individuals experience teasing and put-downs and taking action to interrupt the behavior and/or support the victims.

Code Understanding and Skills (Knowledge In-action)

\section{Workshop \#8. Shifting attitudes/Role playing and skits.}

This is what I asked (NCBI Trainer's Notes as referenced in Brown, 2009, p. 29):

For the final workshop session, I asked the participants to find a new partner and generate a specific list of the kinds of jokes, remarks, and slurs that they hear, and reflect on how 
would they like to be more effective in shifting the attitude of the person making the racial remark. Participants were asked individually to come before the workshop with their listed comment or joke.

This is why I asked it: (1) to demonstrate effective strategies through modeled role play and skits the use of new prejudice reduction workshop skills in order to shift the attitudes of inappropriate, mistaken behaviors; (2) to teach some ways of responding to racial remarks or comments that are most likely to change the other person's attitude; (3) to coach participants to air the feelings first and then to learn an effective response; (4) to understand that the tone of the intervention is important as well some tones increase defensiveness, others decrease it; and, (5) to demonstrate intervention through asking questions in order to decrease defensiveness.

This is what I observed and heard: participants were assigned as allies to be up front of the room with their partner who was learning the intervention skill modeled, and trying out the new applied skill in making an effective response in the role play. Each pair was given feedback from workshop participants on how they did in their skits, trying to intervene well in response to the comment or remark.

This is the impact: informed action (delivery of change through collective insight). 


\section{Capacity Building Generated}

Capacity building was generated from the action research project's professional development workshop activities (Brown, 1984; Epstein, 2004, Kotter, 1996). The support group meetings generated a spirit of camaraderie (Bolman \& Deal, 2003) through shared story experiences. As a result, the participants were motivated by each other's speak outs, cheering on one another and engaged to lead group work activities of the diversity workshop modules (Brown, 2009). At the end of the eight workshops, the participants developed a deeper, mutual commitment to the success of the program. Post training data results confirmed an increase in awareness and identity. Informed action provided insight into discussing possible next steps to take for implementation plans. As a proposed plan of action, next steps leaned towards advanced consulting done by the researcher; follow up, as well as coaching and mentoring (Stage 4) of Brown's model (2009).

The researcher as participant observer has found that utilizing the training skills model was a way to build capacity and connect people around the goals of living and breathing diversity as a school leader. Moreover, what I have come to understand through effective strategic planning and coalition building (Hambright \& Diamantes, 2004) is, while building capacity to create major change we must find the common elements of agreement among diverse parties in order to accomplish together what each party cannot

accomplish alone (Brown, 2009; Kotter, 1996; Putnam, Gunnings-Moton \& Sharp, 2009; Senge, 1990; 2000; Senge et al., 1994).

\section{Six Month Follow Up: Reflective Practice}

In February 2013, a brief follow up evaluation was conducted on the eight week diversity leadership skills module training for the participants in the study. The data 
collected was voluntarily completed and responses were stored confidentially and securely online by Survey Monkey. The researcher used unique identification codes to link respondents' answers to the information collected during the time of the eight week training as well as assured all respondents that this code system would not be used to identify them by name or obtain other information about the respondent.

Quantitatively, the responses to follow up questions 1 and 2 were collected from the purposeful sample of training participants who were asked to respond online as well as the option to select multiple choices from the survey's multiple answers provided to the following:

Survey Question \#1. Which module(s) skills have you found useful in your work since you came back from training?

Survey Question \#2. Which areas of skills application have you shared with colleagues?

Additionally, the responses to question 3 using a Likert type scale to measure likelihood with the following choices: 5=To a Great Extent, 4=To a Considerable Degree, 3=Somewhat, 2=Very Little, and 1=Not at All.

Survey Question \#3. Do you feel as though the training content helped you improve your ability to make decisions about the appropriate course of action involving prejudice behavior?

The learning group's six month follow up survey responses for Survey Question \#1, "Which module(s) skills have you found useful in your work since you came back from training?," are illustrated in Figure 4-11. 


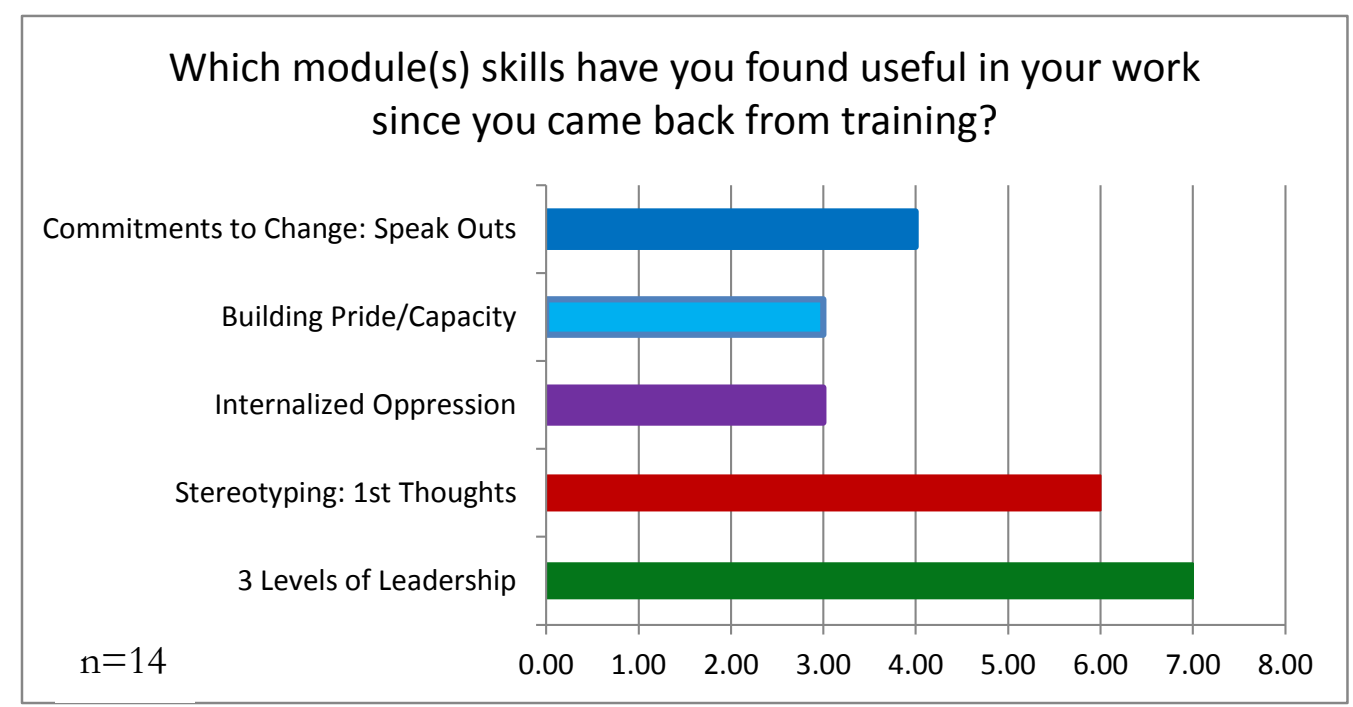

\section{Figure 4-11 Six month follow up Survey Question 1}

Of the available staff, 10 of the original sampled participants $(n=14)$ completed the follow up survey and given the option to select multiple choices from the survey's multiple answers provided. The 3 Levels of Leadership learning module ranked the highest as most useful, and the Stereotyping: $1^{\text {st }}$ Thoughts Exercise module activity ranked next as useful in caucusing work. All other responses included the Speak Outs activity, which ranked third for being useful from training in the commitment to change, while Internalized Oppression as well as Building Pride and Capacity Building exercises both rated last as useful module activities.

The responses to Survey Question \#2, "Which areas of skills application have you shared with colleagues?" are illustrated in Figure 4-12. 


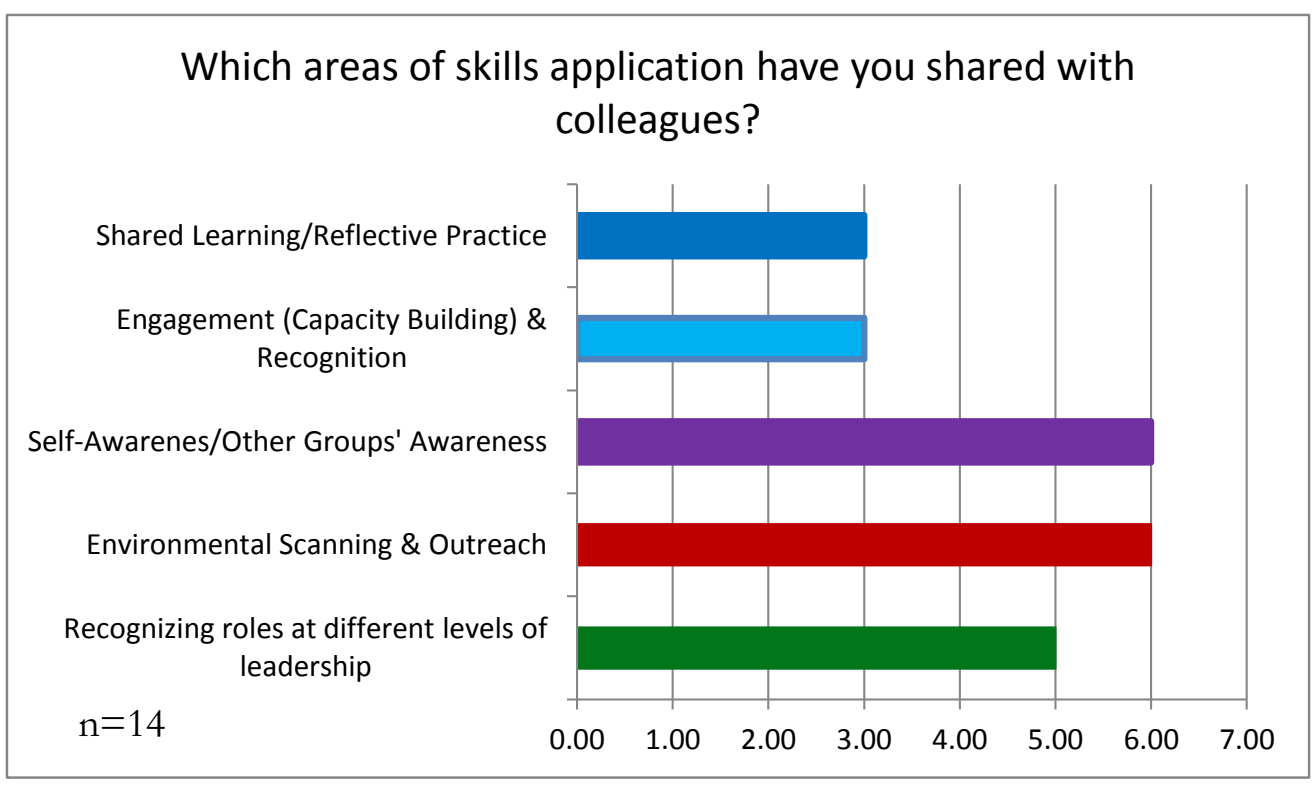

\section{Figure 4-12 Six month follow up Survey Question 2}

Of the available staff, 10 of the original sampled participants $(n=14)$ completed the six month follow up survey. Respondents were given the option to select multiple choices from the survey's multiple answers provided. The skills applications shared most with colleagues involved them helping to create an environment that values and welcomes diversity along with outreach to groups or individuals to ensure inclusion. Being self aware was important in making a conscientious effort through the re-examination of our own records about different groups and individuals that create tension and conflict.

Obtaining new information through ongoing professional development was understood in order to re-educate. Recognizing what roles we have within the school as well as taking different levels of leadership to enhance the school environment for ourselves and others ranked next in shared skills application. Another significant shared skills application collected from the responses was affording individuals the opportunity to 
speak out about their experiences while engaging them in capacity building as well as recognizing individual successes in generating an optimal learning environment that is welcoming for all.

Lastly, the responses to Survey Question \#3, "Do you feel as though the training content helped you improve your ability to make decisions about the appropriate course of action involving prejudice behavior?," are illustrated in Figure 4-13.

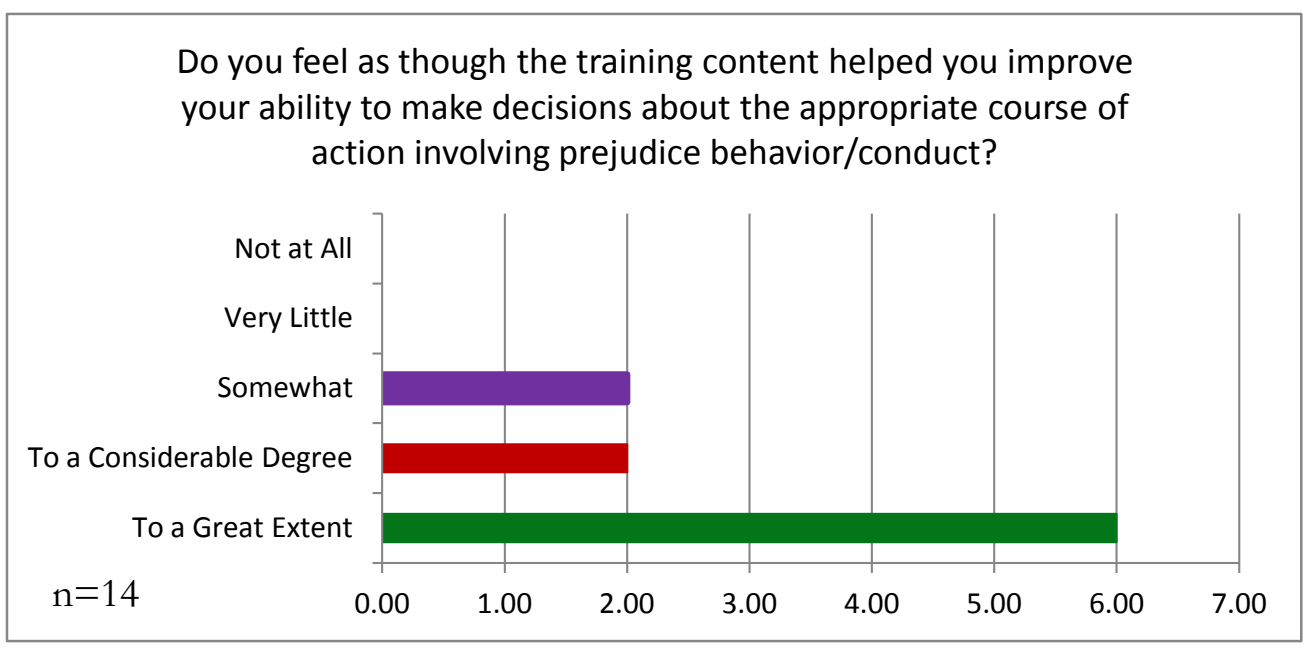

\section{Figure 4-13 Six month follow up Survey Question 3}

Of the available staff, 10 of the original sampled participants $(n=14)$ responded to the six month follow up survey. Most feelings after training found the training's diversity leadership skills content helped to improve their ability to make decisions about the appropriate course of action involving prejudice behavior, rating to a great extent $(60 \%)$. All other responses rated to a considerable degree (20\%) or responded that the training somewhat helped to improve skills (20\%). 


\section{Discussion on Analysis}

The assessed training imparted a better understanding of espoused thoughts and impact on dispositions, giving way to both formative and summative evaluated data. The documentation of coding schemes (Auerbach \& Silverstein, 2003; Creswell, 2007) from interview and observation protocols, reflective journaling and data analysis concluded with the researcher's formulation of personal theories (Compton \& Davis, 2010; Knowles \& Cole, 1996). In addition, the primary research question and its subsidiary questions for analysis were successfully addressed throughout this chapter. Based on the results that emerged from the pre and post assessment survey questions (Likert type), interview and observational protocol data (Creswell, 2007), reflective journaling as well as through the participant evaluated training feedback (Likert type), the data supported the implication that the eight week diversity leadership training and workshop module activities had a positive impact on the sample group. The researcher found reframing strategies helpful, especially engaging those participants who were considered the leaders of the organization.

\section{Conclusion}

The action research training module activities enabled participants to set new goals and used reflective practice strategies to exercise professional autonomy (Epstein, 2004), and strategies with focus on effecting long range organizational change (Bolman \& Deal, 2003; Evans, 1996; Fullen, 2001). Additionally, the significance and essential factors to the success of this collaborative group work were to provide opportunity for participants to identify and to heal the emotional records (Brown, 2009; Haberman, 1994; Purkey, 1992), which in the beginning of the study's activities hindered participants to share their stories and personal reflections. This process enhanced the delivery of change through collective 
insight, which entailed reflecting, rethinking, evaluation, and action plan for implementation.

Consequently, the participants who responded and shared their stories, whether in the workshops or voluntary submission online, affirmed that the professional develop training received was important to them personally and professionally. When given the option (on the participant learning reflections submission) to rate their overall training experience and to choose the level of importance to which the diversity leadership training and skills application helped them, most of the participants responded with "Essential" or "Very Important," communicating their overall satisfaction with the diversity leadership training.

In summary, a formal means of assessment in particular for this organization to adequately collect data and document findings in a more valid manner was long overdue; specifically so, linked to measuring the impact of diversity leadership training skills on prejudice reduction. The leadership training skills and the practiced application for respecting diversity were observably essential for generating a caring school environment as well as encouraged participants' social awareness and involvement. The qualitative data presented in this chapter strengthened the assumption that the participants deemed the diversity leadership training worthwhile and beneficial, particularly in priority area of focus on behavior and conduct. This was observably evident through the administered interview and observation protocols, the qualitative responses from shared stories and reflections in workshops as well the online volunteer participant learning reflections.

Additionally, the illustrated bar graph figures provided an effective visual report, imparting a better understanding of espoused thoughts and impact on dispositions at study 
conclusion. All of the collected and compiled triangulated qualitative data enhanced the quantitative results; thusly, facilitating validity in which all combined methods working together produced a complete research study. Likewise, my expectation of the participants was to acquire a better understanding of prejudice reduction strategies as demonstrated through observational protocols (Creswell, 2007) and to ascertain that understanding from shared reflective learning assignments (Compton \& Davis, 2010) along with the post assessment training surveys. Thus, credibility and validity was qualitatively significant with the use of multiple sources of collected data (Golafshani, 2003; Mills, 2003 \& 2011).

Consequently, this study was culturally sensitive in approach (Tillman, 2002) and was aimed to look at the observed impact of the professional development training on adult participants and disposition toward sustained change in practice as well as whether or not effective skills application result in evidence of change (Kotter, 1996) over time. In addition, the beliefs and attitudes expressed were not necessarily representative of the entire population. Due to this limitation, the conclusions drawn from this cyclic process presented was considered as indicators of successes and needs for improvement. Furthermore, opportunity for growth based on the concluding findings was suggestive as beneficial to the school improvement planning process. However, it is the belief of the researcher that these findings could be a valuable resource to guide future explorations into program school improvement and to gain an understanding of the institution's strengths with a continuum action plan.

Likewise, theories of the controversial issues process concerning prejudice reduction actions taken as presented and referenced by the researcher sought to relate informal and formal methods of diversity skills training. The significance of study and its 
implications was valuable to the organization's levels of leadership in considering self awareness, others awareness in being an ally, and the agency's awareness for taking action. By doing so, these actions that were taken helped with building capacity to create major change (Epstein, 2004; Gephart \& Marsick, 2003; Waltzer, 1987 as referenced in Freeman, 2010). Reed (1996) agrees with this method of building capacity (Brown, 2009; Epstein, 2004; Gephart \& Marsick, 2003; Waltzer, 1987 as referenced in Freeman, 2010), within the school environment in helping to develop awareness of one another's untapped potentials (Reed, 1996, p.81).

Furthermore, prejudice reduction practices and their application for respecting diversity are essential in creating a safe and welcoming professional learning community for all its members. Prejudice reduction is a positive process whereby individuals resolve issues in an informal or formal atmosphere, or where issues are resolved as part of the ongoing interaction between individuals. Folger, Poole, \& Stutman (2001) argue that "Social confrontation episodes involve conflict over conduct and rules of conduct" (p.99). Misinterpretations and obscure behaviors complicate the relationship between various groups within the learning communities. These misunderstandings are the result of diversity in ethnic backgrounds; others are caused by widely adapted generational disparities. Therefore, the action research process is important in analyzing salient factors or the areas of critical concern impacting prejudice and controversial issues in urban education. Once more, these factors to include levels of leadership, the significance of environmental scanning, stereotyping, internalized oppression, building pride and capacity, becoming allies, making commitments to change, and shifting attitudes (Brown, 2009; NCBI, 2010 \& 2011) are all factors to consider in social justice leadership. 
The authors (Carnall, 2008; Dawson, 2010; Stephen, 2010; Tidd, 2010) maintain that change agents may be either external or internal. Nevertheless, the success of any organizational change paradigm relies significantly on the quality and workability of the relationship between the change agent and the primary stakeholders within the organizational system (Burke, 2011). Moreover, building a caring school environment brings all stakeholders (Epstein, 2004; Freeman \& Vasconcelos, 2010; Gephart \& Marsick, 2003; Waltzer, 1987 as referenced in Freeman, 2010) together from all parts of the school community as a cohesive team. The study in part enhanced the quality of the education programming for optimal student learning as well as encouraged community awareness and involvement. The observed workshop module activities evidenced that the participants developed a collective insight and informed practice strategies necessary to enhance their personal and professional development. This dispositional change was evident when the participant changed their communication style from one that communicated lack of understanding and arrogance towards a parent, to one that communicated empathy and acceptance.

Equitably united, school leaders as well as stakeholders serve as allies within the school environment and its community. This effectively enhanced leadership framework can then be used to ameliorate prejudice, handle controversial issues, and negating situations surrounding diversity in educational leadership. It is the researcher's belief that the action research project itself would be an essential resource tool used to solidify a delivery of change, utilizing a selected group sampling to conduct the study. Hence, the "organization ha[d] desired outcomes reflected in its vision, mission and [core] values" (Aguilar, et. al., 2004, p. 40). Moreover, this vision or "a mental image of a possible and 
desirable future state of the organization" (Bennis \& Nanus, 2003, p.82) is symbolically paramount for the attainment of a group structure's desired purpose. Lastly, building emotionally intelligent organizations (Goleman, 2002) involved collective input into the vision.

Moreover, concluding findings presented implications towards a proposed change initiative for the school environment, focusing on prejudice behavior as the determined priority area, including the frequency and type of inappropriate behavior. This exploratory study gave some discovered insight with supported theoretical and conceptual strategic approaches that demonstrated understanding of the paradigm frameworks on organizational change, which concluded as evidenced by the study data, seeing change as an opportunity rather than an impediment. The researcher observed firsthand how the module exercises had an overwhelmingly empowering effect upon the majority the study's participants who took part in the change initiative on leadership for diversity in this study. I was able to compile and review significant output data for quantitative analysis purposes, and if required by the educational agency, for other reporting purposes if needed later.

Lastly, educational leaders who support an intellectual, emotional, and supportive learning environment generate "an atmosphere where people feel they are stretching and growing while enhancing their ability to perform" (Aguilar, Mantel, Maslowski, McDaniel \& Miller, 2004, p. 122). Goleman (2002) points out further that relationship management strengthen employees' capabilities and generates a sense of self worth through administrative feedback and guidance. Just as reflective practice gives administrators profound insight of their best practices in education and classroom management, Schön (1974) maintains that the benefits of reflective practice can help you to recognize your 
strengths and opportunities. The researcher believes that this reflective method and informed practice are beneficial to enhancing one's leadership skills as well as helping educational leaders to effectively address in application with situational decisions faced in the social justice issues process to address diminishing prejudice in the workplace, especially in educational environments, because of the influence educational environments have on learning, growth, and professional development. 


\section{Chapter 5}

\section{Conclusions and Implications}

At the beginning of this action research study, my objective was to measure the impact of diversity leadership training skills from the dispositions of a sample of the participants for the purpose of conducting professional development that would diminish reduction in the school and classroom. Specifically, the researcher wanted to assess the impact of Brown's (2009) modeled diversity the leadership training and the researcher's organized professional development module activities had, focusing on the areas of behavior and conduct. Additionally, I sought to understand how to improve the school environment in order to find out how prejudice behavior can be reduced. The action research study's inquiry and formative assessment processes were fulfilled in order to understand what factors promoted changes in reducing prejudice behaviors and or unconscious practices; whereby, instructional leaders could apply the leadership training skills in building a caring school environment and enhanced leadership framework at all levels of the organization: central administration, site administration, and instructional in the classroom where employees at various levels within the organization had equal status with different responsibilities and roles. Thus, the leadership framework created collectivity of purpose in prejudice reduction. Lastly, my goal to seek a better understanding on how capacity building was considered necessary for organizational change was achieved to learn how training's effective impact benefited all levels of leadership.

Similarly, the research data suggests that by adapting such a model as Brown's (2009) schools will be able to effectively conduct social justice work in the controversial issue process within the professional learning communities. This practiced model as 
referenced is suggestive as a possible learning framework resource for urban education school administrators. Nevertheless, the researcher believes there is a window of opportunity based on the receptiveness to what the school learning community can offer through such practiced prejudice reduction models as in the adaptive model used for this study and other multicultural curriculum programs.

The follow up responses from the sample of participants, who exercised the module activities and completed their professional development over the summer of 2012, were used to help determine the diversity leadership training's success. This chapter provides a summary of the overall study, including what was accomplished, the research limitations, recommendations for suggestive delivery of change, implications, and directions for future research study on informed practice and organizational change on professional development and prejudice reduction. Other pertinent information such as reflections on importance of study as a participant observer, as well as, what the study revealed, and why study mattered will be shared in the conclusions section of this chapter.

\section{Summary of the Findings}

Successes in planning, assessment, observation, and reflection provided study participants with evidence that enabled them to feel capable and competent, thus motivated them to continue to invest in their personal and professional development in registering for the November 2012 and April 2013 leadership conferences. The diversity leadership training was found to have an integral part in the development of higher order thinking that enabled understanding through the successful shared stories and learning experiences in the series of diversity workshops. The researcher found reframing strategies helpful, especially engaging those participants who were considered the pioneers of the organization. By 
providing participants with the module activities to include caucusing activities, the use of speak outs of personal story, role play exercises, and writing reflections, resulted in deep thinking and learning took place. This study provided needed details relevant to Kotter's (1996) theoretical approach of change framework, indicating that when information moves throughout the organization, rather than simply assisting in disseminating the information, each participant had an effect on the data, information, knowledge, and wisdom obtained through the professional development to reduce prejudice. Likewise, using Kotter's eight stage process (1996, p.21) showed some significant evidence of change for the educational organization's individuals that went through the workshops and participated in the study. In that, this approach was helpful to encourage shared learning initiatives through the professional development in alignment to organizational change as well as building capacity (Epstein, 2004; Fullen, 2001; Gephart \& Marsick, 2003; Muhammad, 2009).

As participant observer, the researcher found that diversity leadership training skills work best when we as instructional leaders learn about the painful internalized feelings within each group in our school environments. What was learned through the observed training modules facilitated is personal and professional development that advanced a greater appreciation and tolerance for the struggles of groups within the 14 participants. They were able to become more engaged and generated a higher awareness in overall leadership as educators. As participant observer, I noticeably found it helpful to know that during the course of the eight week professional development workshop activities, some people found it very easy to talk about the things they could not stand about their group. However, in hearing what others shared in group dialogue, some were taught that you never air negative feelings about your own group in public. For example, a black male, 
Board Member, expressed his concern saying "It is not a good idea to say the things we cannot stand about our own group within a mixed group, because it will reinforce the stereotypes.” As the participant observer leading the workshops, I explained stating that those concerns are understandable, but this researcher's experience from train-the-trainer conferences is that exactly the opposite happens. For some groups, it appeared difficult to say what they could not stand about members of their own group as part of a module activity, because they feared that others would hear these words and simply have their own prejudices reinforced.

Moreover, I found through my direct observations that when people get a chance to see the impact of internalizing stereotypes they gained an even greater appreciation of the struggles of each group which is essential as an educational leader. The observed lessons learned found that participants realized that everything heard about or have been told about another group is internalized, forming a literal 'record' inside us (Brown, 2009). As explained through the series workshops and module activities, Brown (2009) concludes that even when our thoughts and experiences refute these records, they still exist and influence our behavior. Therefore, we are all born innocent, a blank slate. No one chooses to have these prejudicial ideas. However, we all have them about other groups as evidenced and realized through the training modules. The training session exercises provided a chance to get the stereotypes and prejudicial ideas out into the open and get rid of them.

As a result of the eight week professional development training, participants came away with the understanding how similar our struggles really are with insight as to how we interact with each in our diverse learning communities. This study helped with the understanding that many of the things we cannot stand about our own groups are simply 
internalizations of what people from outside our groups have said about us, about others, and or about the community in which we live. Lastly, the observed realization discovered is that the things we hate about our own groups are the scars our groups carry from being mistreated. The building of relationships or building the capacity for learning and change (Brown, 2009; Gephart \& Marsick, 2003) is vital to understand the issues to effectively reduce prejudice behavior and to create, where it is feasible, a climate of cooperative cohesiveness. Thusly, the research suggests that we strengthen and enhance a leadership framework for promoting an effective learning environment for all within the educational organization.

Lastly, the researcher's intent was to present theoretical knowledge that demonstrated understanding of the conceptualized framework on organizational change with descriptive specific actions taken and a suggested design of delivery in an action plan for school improvement. Prejudice reduction is a positive process whereby individuals resolve issues in an informal or formal atmosphere, or where issues are resolved as part of the ongoing interaction between individuals. As participant observer, my objective was to point out significance, and the priority area of focus was to target success at reducing prejudice behavior and build capacity for improved conduct in the school environment. The action research process and its suggestive paradigm approach of activities for this priority area aim was to reflect change as an opportunity rather than an impediment in the school environment. 


\section{Implications}

\section{Theory and Research}

Among the implications of this study on theory are its contributions to the cyclical and spiral experiential learning framework (Knowles \& Cole, 1996) of action research, indicating the effects of collaboration and success on motivation (Brown, 2009; Kotter, 1996) as evidenced in this research study. Among the implications for research is the need to investigate the direct effects of time, reflection, and discussion on diversity in educational leadership training. Secondly, I recommend that this study be replicated $\mathrm{K}-16$ as well as in other educational endeavors. Lastly, there is the need to conduct a longitudinal study in this area to determine these elements long term impacts in considering new research questions.

\section{Informed Practice}

Organizational dynamics. Among the implications for informed practice are a greater understanding of the elements at work in catalyzing diversity leadership training, including the effects of success, as well as the environments and organizational dynamics required to encourage deep thinking and learning on issues of prejudice reduction and leadership development in all levels of various positions and statuses of employment, such as educational administrators, teachers, teacher assistants, and other school personnel. Additionally, peer assessment like in action research collaboration gives way for enhancing and stretching cognitive abilities to generate higher order leveled thinking and critical thinking skills. The conclusions drawn from this cyclic process were considered as indicators of successes and needs for improvement based upon findings to include suggestions for directions on future study. 
Metacognitive processes. According to Wiggins and McTighe (2007), successful learning requires metacognition; whereas, the individual learns how to reflect, self assess, and use feedback to self adjust. The authors agree that these metacognitive processes can (and should) be taught explicitly (Wiggins \& McTighe, 2007); thus, learning by doing (DuFour et al., 2006; Knowles \& Cole, 1996). Therefore, peer assessment is another means of promoting effective learning and instruction practices. Again, this process "encourages [participant] autonomy and higher order thinking skills" (Bostock, 2006, p. 1).

Teaching practice. As a result of this study, what I have learned is that the method of inquiry, or rather the goal of using questioning and listening strategies is to gather as much information as possible in order to inform practice reflectively as an educator. Specifically, the study's group work module activities demonstrated that Emotional Intelligence (EQ) and reflective practice show a parallel relationship in association with relationship management, social awareness, and self awareness (Goleman, 2002, p. 39). First of all, teamwork and collaboration as it relates to cooperation and teambuilding draw parallel to reflective practice by it being "a strengths-based, mutually respectful and collaborative approach to supervision. It presents an environment of intellectual inquiry... [and] emotional safety" (Aguilar, et. al., 2004). Secondly, social awareness as it relates to empathy shows a relationship to reflective practice by generating or providing an empathetic environment for subordinate organizational members. Listening takes part in this process and improves interpersonal relationships; whereby, demonstrating and expressing understanding as well as acknowledging subordinate organizational members. Lastly, self awareness as it relates to a sense of self confidence in capabilities apply to 
reflective practice in leadership by utilizing your "knowledge to enhance professional competence" (Aguilar, et. al., 2004).

\section{Policy}

This study proposes that school reform leaders should focus on maintaining alignment to the school mission. This requires not only a periodic review of the mission statement, and a possible update but a leadership exploration of everyone in the organization to be open to, prepared for, and willing to implement the new mission. In this study, the new mission required prejudice reduction. No matter what the requirements may be to implement a new mission, there will always be requirements that need to be addressed. In addition, school reform leaders should focus on being prepared for critical changes that one may face to help improve the overall school environment at all levels of leadership. Reflectively, what this researcher has learned from this study and recommends is that coalition builders attempt to understand the interests of all parties concerned about an issue and to create, where it is feasible, and a climate of cooperative cohesiveness. Lastly, it is recommended that policy decision makers use collaborative learning environments to support ongoing professional development for all levels of employment within the educational agency.

\section{Capacity Building}

Furthermore, while building capacity to create major change we must find the common elements of agreement among diverse parties in order to accomplish together what each party cannot accomplish alone (Brown, 2009; Kotter, 1996; Putnam, GunningsMoton \& Sharp, 2009; Senge, 1990; 2000; Senge et al., 1994). Capacity building according to Epstein (2004) involves stakeholders' systems thinking at all levels: at Board level, 
learning to solve problems and manage dilemmas; at the Superintendent level, being better political, managerial, school administrators; at school level, practicing principals who serve in the capacity of instructional leaders, and curriculum supervisors; and lastly, at classroom level, as professional educators and teaching experts in knowledge and skills (p.122).

\section{Limitations}

Focus group selected represented only a purposeful sample of the total participants in the program. Recruitment of subjects in this manner insured that all viewpoints were adequately represented in addition to sample selected included respondents at both ends of the spectrum, as well as, some in the middle who had the best knowledge and experience to include novice educators, a volunteer Board member, central office, and site administration. The resulting purposeful sample members selected to comprise focus group were densely interconnected; whereby, snowball or chain referral sampling techniques was deemed best appropriate for the focus group to minimize bias while maintaining privacy and confidentiality (Marshall, 2003).

Furthermore, the beliefs, dispositions, and attitudes expressed were not necessarily representative of the entire population. Due to this limitation, the conclusions drawn from this cyclic process should be considered as indicators of successes and needs for improvement based upon findings reported. Lastly, the data collected served to inform diversity leadership training effectiveness with respect to addressing the research questions. Statistical significance was not applied as a measure in considering sampling method used as well as results found from data obtained was reported in terms of their actual frequencies. 


\section{Recommendations for Informed Practice}

Prejudice reduction is a positive process whereby individuals resolve issues in an informal or formal atmosphere, or where issues are resolved as part of the ongoing interaction between individuals. A parallel conversion plan presented (See Appendix K) is indicative as a delivery of change in monitoring implementation for articulating the connections between new behaviors and organizational success. This plan is the use of both systems simultaneously to ensure the new system works correctly. As staff is trained and phases are successfully evaluated, old system will be shut down. Furthermore, this subsequent plan would provide a developed means to ensure leadership development and succession. The implementation process builds on the school improvement plan change initiatives. The resulting outcomes from the change initiatives impact on prejudice reduction will then produce supporting data and information as measurable evidence of success based on documentation, new operating procedures, and communication system.

\section{Directions for Future Research Study}

According to Paluck \& Green (2009), their review places special emphasis on assessing the methodological aspects of intervention specifically on research design and measurement. The authors contend that some intergroup contact and cooperation interventions appear promising; however, a more thorough an expanded empirical assessment of prejudice reduction strategies is needed to determine what works. Suggestive areas of further inquiry and discussion may perhaps look at ethical and pedagogical issues, in particularly, the impact of informed practice on children's training, and or the long range effects in measuring the impact of diversity leadership training skills on prejudice reduction, specifically as it pertains to informed practice and professional development. A 
longitudinal study would perhaps help to inform educational policy makers and practitioners who may undertake facilitating group research collaboration work, involving ethical and pedagogical issues. Likewise, conduct a more in depth assessment of diversity leadership training in regards to parental involvement. Consequently, this approach may generate inquiry for a new research question: What long range effects of prejudice reduction practices have been proven positive to the efficacy of a diversity leadership framework?

\section{Reflections on Importance of Study}

As this study progressed, the researcher began to realize how vital this educational organization had been to the stakeholders involved in this study as well as to the extended community. Additionally, this educational organization was a major professional landmark in the researcher's early childhood education career through past and professional experiences. The agency's initial influenced vision was an example of community commitment that was pursued and achieved for the targeted African American community. In addition, it was through this organization's initial employment and diversified work experiences for nearly 25 years of professional development, the researcher had acquired attainable practiced skills, experience, and knowledge in the area of public administration and educational leadership. This organization saw my potential growth and maturity as an individual of positive character and provided me with opportunity for professional development.

As participant observer, the researcher was connected to agency's mission reassessment. The study became a vested interest for me professionally as well as for stakeholders in considering systems thinking and school improvement. The outcome results 
were beneficial in the strategic planning process. As an early education practitioner and school administrator, the researcher discovered that the implications of organizational change suggest the understanding that changes can have complex ripple effects across the system impacting professional autonomy. What the change theorists (Bolman \& Deal, 2003; Evans, 1996; Fullen, 2001; Kotter, 1996) emphasize is primary on how individuals and organizations can deal with change successfully and develop appropriate strategies and structures for the effective management of change; thus, taking into account the decision making process and or shared governance (Epstein, 2004). Accordingly, the management of change must provide guidelines for institutional and structural change as well as for individual (Bolman \& Deal, 2003; Evans, 1996; Fullen, 2001).

\section{Conclusion}

Why does this study about the impact of diversity leadership skills training matter? It matters, because "when you change what you believe, you change what you do" (Johnson, 1998). Social learning theorists, according to Dr. Kay Lovelace Taylor (2004) suggests that "that our behaviors are a reflection of what we believe and our beliefs are based on our past experiences combined with our emotional and cognitive internal state" (Huitt, 2001 as referenced in Taylor, 2004, p. 11). Taylor maintains that as educators we understand our individual weaknesses and have the ability to "articulate in discussing the causes for [our] successes and failures" (p. 101). What the researcher has discovered in learning from these situational and leadership perspective group activities are "that any group, regardless of setting, must address itself to the successful completion of a task" (Tuckman, 1965, as referenced in Wren, 1995, p. 355-356). Goleman (2002) discusses about the role of the leader in developing emotionally intelligent and high functioning 
teams. With this perspective in mind, it is the researcher's findings that group task activities and "[r]oles become flexible and functional, and group energy is channeled into the task" (Wren, 1995, p. 359). Hence, the study's participants were able to successfully perform by resolving the organization's structural issues, specifically so in the educational organization's mission reassessment.

As learned from this particular study, diversity in educational leadership helps you to address challenges by utilizing various leadership strategies and modes of communication to effectively apply and implement a plan of action based on situational experiences encountered. An effective leader is more so directed by integrity through the trust of others, openness with sincerity or honesty towards others, and credibility in being the person of your word with owning up to your proposed works and deeds. With that thought in mind, influencing stakeholders within the school community is critical, generating a community partnership of allies to assist with developing strategies for enhancing the academic progresses of the children taught and served. This collaboration of human resources can be instrumental emphasizing the influential presence as the educational leader.

Likewise, school reform leaders should focus on maintaining alignment to the school mission and being prepared for critical changes that one may face to help improve the overall school environment at all levels of leadership. Reflectively, what this researcher has learned as an education practitioner and school administrator is that coalition builders attempt to understand the apprehensions of all parties concerned about an issue and to create, where coalition building is feasible, a climate of cooperative cohesiveness. Such is 
as collaborative learning environments to support ongoing professional development for all levels of stakeholders.

Furthermore, this research study's theoretical practices in leadership along with applied prejudice reduction communication skills provided participants with a better understanding on how to carry out situational decisions such as in the reduction of prejudicial behavior in the school environment. The acquired knowledge attained and shared learning experiences were modeled, focusing on the identified issues of leadership to enhance an individual's skills as a better leader. Studying leadership theoretical practices will help enhance empowerment abilities as a leader who in turn creates opportunities of shared governance (Epstein, 2004) among team players and stakeholders. Similarly, Polizzi (2007) communicates this ideology, referencing Mills (1959). He asserts that "you use your life experience in your intellectual work: continually examine and interpret it. In this sense, craftsmanship is the center of yourself and you are personally involved in every intellectual product upon which you work" (Mills, 1959, p.196 as quoted in Polizzi, 2007, p. 22). By doing so, this action would contribute to the success of the educational community in obtaining those potential allies while increasing the overall resource capacity to diminish prejudicial behaviors and policies within the school.

Inasmuch, educational leaders can facilitate and model how adult learners view and respond to the world by creating a welcoming learning environment that provides equity, and reflects, and embraces the diversity in which we live. A multicultural curriculum lays the foundation for a lifetime. In considering that this study's action research project was conducted in an early childhood education agency, best practices in early childhood education is appropriate to help students early on through exploring ways to "contradict" 
and to interrupt the internalization of negative stereotypes on all groups. Observably, the training module activities engaged participants where they began to become aware of their own internalized racism through some "event or series of events that force[d] the...person to acknowledge [its] personal impact” (Tatem, 1997, p. 55). The participants worked actively to learn about and affirm their own racial identity. Throughout the study, they were able to establish a positive personal identity for themselves as well as others' awareness.

In closing, this study matters because the magnitude of change lies in the eye of the beholder. Dr. King stated, "Our lives begin to end the day we become silent about things that matter" (King \& Washington, 1992). Inasmuch that same change may have different implications for different stakeholders in considering professional autonomy as well as multidirectional accountability and shared governance (Epstein, 2004). What this researcher identifies with as an educational practitioner and school administrator is the implication that leaders need to understand whether changes are first or second order for stakeholders to differentiate their leadership styles accordingly conducive to the decision making process in regards social justice and address diminishing prejudice in the educational environment that impacts on learning, growth, and professional development. The study in part enhanced the quality of the education programming for optimal student learning as well as encouraged community awareness and involvement. Lastly, the study added to the research literature in professional development training of prejudice reduction among school personnel. 


\section{References}

Aguilar, C.R., Mantel, M.J., Maslowski, L., McDaniel, D. Miller, C.J. (2004). The nonprofits' guide to the power of appreciative inquiry. Denver, CO: Community Development Institute.

Argyris, C., Putnam, R., \& McLain Smith, D. (1985). Action science: Concepts, methods, and skills for research and intervention. San Francisco: Jossey-Bass.

Argyris, C. \& Schön, D.D. (1974). Theory in practice: Increasing professional effectiveness. 2nd Ed., San Francisco: Jossey Bass.

Assessments (surveyed participants via Survey Monkey), interviews (Board Members \& Program Administration), observational protocol data, and electronically submitted participant learning reflections' data were administered and collected from agency personnel and administrators from representing center locations within the Black People's Unity Movement (BPUM) Child Development Centers, Inc. and its parenting agency BPUM Impact Corporation in Camden, New Jersey. June 2012 - March 2013.

Auerbach, C. \& Silverstein, L. B. (2003). Qualitative data: An introduction to coding and analysis. New York: NYU Press.

Banks, J.A. \& Banks, C.A.M. (Eds.). (1995). Handbook of research on multicultural education. New York: Macmillan.

Bennis, W. \& Nanus, B. (2003). Leaders: Strategies for Taking Charge. New York, NY: Harper Business Essentials.

Berlin, I. (1936). Let's Face the Music and Dance. Song written for the Hollywood musical comedy film Follow the Fleet. Directed by Mark Sandrich with script by Allan Scott and Dwight Taylor.

Bogdan, R., \& Bikklen, S. (2007). Qualitative research for education. An introduction to theories and methods (5th Ed.). Boston. Pearson Education, Inc.

Bolman, L.G. \& Deal, T.E. (2003). Reframing organizations: Artistry, choice, and leadership. San Francisco: Jossey-Bass.

BPUM Parent Handbook. (2003). Policies, rules, and regulations. Revised April 1, 2003.

Brown, C.R. (1984). The art of coalition building: A guide for community leaders. New York: The American Jewish Committee. 
Brown, C.R., Mazza, G.J. \& NCBI. (1998). Healing into action: A leadership guide for creating diverse communities. Washington, D.C.: National Coalition Building Institute.

Brown, C.R., Mazza, G.J. \& NCBI. (2005). Leading diverse communities: A how-to guide for moving from healing into action. San Francisco, CA: Jossey-Bass.

Brown, C. R. \& National Coalition Building Institute Professional Leadership Conferences (2009; 2010 \& 2011). Leading the NCBI Diversity \& Inclusion Workshop (Learning Track \#1); Skills for Effective Diversity \& Inclusion Leadership__"The Diversity Savvy Leader” (Learning Track \#2); Violence Prevention Workshop. National Coalition Building Institute on Leadership for Diversity Institute conference proceedings. Hunt Valley, MD. November $4-8$, 2009; November 3 - 7, 2010; November 2 - 6, 2011.

Brown, R. \& Carbonari, J. (1977). Evaluation of a multi-cultural project designed to reduce group isolation. Paper presented at the $61^{\text {st }}$ Annual Meeting of the American Educational Research Association. New York, NY. April 4 - 8, 1977.

Bolman, L.G. \& Deal, T.E. (2003). Reframing organizations: Artistry, choice, and leadership. San Francisco: Jossey-Bass.

Bostock, S. (2006). Student peer assessment. The Higher Education Academy.

Burke, W. W. (2011). Organizational change: Theory and practice. Thousand Oaks, CA: Sage.

Butt, K.L. \& Pahnos, M.L. (1995). Why we need a multicultural focus in our schools. JOPERD--The Journal of Physical Education, Recreation \& Dance, 66.

Calhoun, E.F. (1994). How to use action research in the self-renewing school. Alexandria, VA: Association for Supervision and Curriculum Development.

Carnall, C. (2008). Managing change in organizations. Upper Saddle River, NJ: Prentice Hall.

Carpenter, S., Zárate, M.A. \& Garza, A.A. (2007). Cultural pluralism and prejudice reduction. Cultural Diversity and Ethnic Minority Psychology, 13(2), 83-93.

Choi, E. (Ph.D.). (2010). A case study of an early childhood inclusive program: Teacher professional development and collaboration. A dissertation in Curriculum \& Instruction. (Unpublished doctoral dissertation). Pennsylvania State University.

Clark, D.R. (2004), Performance typology map. Retrieved from http://www.nwlink.com/ donclark/performance/performance_typology.html 
Compton, L. \& Davis, N. (2010). The impact of and key elements for a successful virtual early field experience. Contemporary Issues in Technology and Teacher Education, 10(3). Retrieved from http://www.citejournal.org/vol10/iss3/general/article1.cfm

Craig, D. V. (2008). Action research essentials. San Francisco, CA: Jossey-Bass.

Creswell, J. W. (2003). Research design: Qualitative, quantitative, and mixed method approaches (2nd ed.).Thousand Oaks, CA: Sage Publications.

Creswell, J. W. (2007). Qualitative inquiry and research design: Choosing among five approaches. Thousand Oaks, CA: Sage Publications.

Creswell, J. W., \& Plano-Clark, V.L., (2011). Designing and conducting mixed methods research. Sage Publications, Inc.

Creswell, J.W. \& Tashakkori, A. (2007). Editorial: Differing perspectives on mixed methods research. Journal of Mixed Methods Research, 1(40), 303-308.

Davis, Ricardo. (1988). The largest Camden County employers. New Jersey Directory. Philadelphia Business Journal, 7, $12 B$.

Dawson, P. M. B. (2010). Managing change, creativity and innovation. Thousand Oaks, CA: Sage.

Denzin, N.K. \& Lincoln, Y.S. (Eds.). (2005). The sage handbook of qualitative research (3rd ed.). Thousand Oaks, CA: Sage Publications.

DuFour \& DuFour. (2010). The Importance of Short-Term SMART Goals. Solution Tree Press.

DuFour, DuFour, Eaker, \& Many. (2006). Learning by doing: A handbook for professional learning communities at work. Solution Tree Press.

Epstein, N. (Ed.). (2004). Who's in charge here?: The tangled web of school governance and policy. Washington, D.C.: Brookings Institution Press.

Evans, R. (1996). The human side of change. San Francisco, CA: Jossey-Bass.

FirstClickSEO, LLC. (2007). Conversions. Retrieved from, http://www.firstclickseo.com/services/seo-process.html

Freeman, M. (Ed.). (2010). Critical social theory and evaluation practice. New Directions for Evaluation, 127. Jossey-Bass \& American Evaluation Association. 
Freeman, M. \& Vasconcelos, E.F.S. (2010). Critical social theory: Core tenets, inherent issues. In M. Freeman (Ed.), Critical social theory and evaluation practice. New Directions for Evaluation, 127, 7-19.

Folger, J.P., Poole, M.S. \& Stutman, R.K. (2001). Working through conflict: Strategies for relationships, groups, and organizations. $\left(4^{\text {th }} \mathrm{ed}\right.$.). New York: Longman.

Fullen, M. (2001). Leading in a culture of change. San Francisco, CA: Jossey-Bass.

Gay, L.R., Mills, G.E., \& Airasian, P. (2009). Educational research: Competencies for analysis and application ( $\left.9^{\text {th }} e d.\right)$. Upper Saddle River, NJ: Merrill/Prentice Hall.

Gephart, M.A. \& Marsick, V. J. (2003). Introduction to special issues on action research: Building the capacity for learning and change. Human Resource Planning, 26, 2.

Gimmestad, B.J. \& De Chiara, E. (1982). Dramatic plays: A vehicle for prejudice reduction in the elementary school. Journal of Educational Research, 76.

Goleman, D., Boyatzis, R. \& McKee, A. (2002). Primal leadership: Realizing the power of emotional intelligence. Boston: Harvard University Press.

Golafshani, N. (2003). Understanding reliability and validity in qualitative Research. The Qualitative Report, 8(4), 597-607. Retrieved from, http://www.nova.edu/ssss/QR8-4/golafshani.pdf

Haberman, M. (1994). Preparing teachers for the real world of urban schools. The Educational Forum, 58, 162-168.

Hambright, G. \& Diamantes, T. (2004). An analysis of prevailing K-12 educational strategic planning models. Education (Chula Vista, Calif.), 125(1), 97-103. Retrieved from Education Full Text database.

Henze, R. (2001). Leading for diversity: How school leaders can improve interethnic relations. Educational Practice Report 7. Center for Research on Education, Diversity and Excellence. Santa Cruz, CA.

Howard, G. (1985). Project REACH: Teacher guide and training manual. Arlington, WA: REACH Center.

Huff, J.K. (Ed.D.). (1998). The influence of media in the disclosure of racial attitude. (Published doctoral dissertation). University of South Dakota.

Huitt, W. (2001). Social cognition. Educational Psychology Interactive. Retrieved from, http://chiron.valdosta.edu/whuitt/col/soccog/soccog.html. 
Huntjens, P., Termeer, C, Eshuis, J., \& Van Buuren, M.W. (2011). Collaborative action research for the governance of climate adaptation - foundations, conditions and pitfalls. Key Deliverable 1A, Theme 7: Governance of adaptation. Dutch National Research Programme Knowledge for Climate, 2011.

Johnson, Spencer (M.D.). (1998). Who moved my cheese?: An a-mazing way to deal with change in your work and in your life. Foreword by Kenneth Blanchard. New York, NY: Putnam Adult.

Kemmis, S. \& McTaggart, R. (Eds.) (1988). The action research planner, ( ${ }^{\text {rd }}$ ed.). Victoria, Australia: Deakin University Press.

Kemmis, S. \& McTaggart, R. (2000). Participatory action research. In N.K. Denzin \& Y.S. Lincoln (Eds.) Handbook of qualitative research (2nd ed.) (pp. 567-605) Thousand Oaks: Sage.

King, Jr., M.L. \& Washington J. (Ed.) (1992). I have a dream: Writings and speeches that changed the world. Forward by Coretta Scott King. Harper One Publishing.

Klein, T.E. (1992). Teaching tolerance: Prejudice awareness and reduction in secondary schools. A thesis presented to the faculty of the Dominican College Department of Education in partial fulfillment of the requirements for the degree of Master of Science in Education: Curriculum and Instruction. (Unpublished doctoral dissertation). San Rafael, California.

Knowles, J. G., \& Cole, A. L. (1996). Developing practice through field experiences. In F. Murray (Ed.). The teacher educator's handbook. Building a knowledge base for the preparation of teachers. San Francisco, CA: Jossey-Bass.

Kotter, J. (1996). Leading change. Boston, MA. Harvard Business School Press

Kotter, J. P. \& Cohen, D. S. (2002). Creating ways to empower action to change the organization: Cases in point. Journal of Organizational Excellence.

Kupetz, B.N. (Ed.D.). 2008. Do you see what I see? Appreciating diversity in early childhood settings. Earlychildhood NEWS. The Professional Resource for Teachers and Parents. Retrieved from http://www.earlychildhoodnews.com/earlychildhood/article_view.aspx?ArticleID $=147$.

Lutkehaus, N. C. (2008). Margaret Mead: The making of an American icon. Princeton University Press.

Lynch, J. (1987). Prejudice reduction and the schools. New York: Nichols. 
Mandela, N. (2003). Lighting your way to a better future. Speech delivered by Mr. N.R. Mandela at launch of Mindset Network. Launch of Mindset Network. Planetarium, University of the Witwatersrand Johannesburg South Africa. Retrieved from http://db.nelsonmandela.org/speeches/pub_view.asp?pg=item\&ItemID=NMS909 \&txtstr=lighting\%20your

Marshall, C. \& Rossman, G. (1999). Designing qualitative research. ( ${ }^{\text {rd }}$ Ed.). Thousand Oaks, CA. Sage Publications.

Marshall, P.A. (2003). Human subjects' protections, institutional review boards, and cultural anthropological research. Anthropol Q, 76(2):269-85.

McKown, C. (2005). Applying ecological theory to advance the science and practice of school-based prejudice reduction interventions. Educational Psychologist, 40(3), 177-189.

McMillan (2004). Educational research: Fundamentals for the consumer. ( $4^{\text {th }}$ Ed.). Boston, MA: Pearson Education, Inc.

McTaggart, R. (Ed.). (1997). Participatory action research: International contexts and consequences. Albany, NY: State University of New York Press.

McKinsey \& Company (2001) Effective capacity building in nonprofit organizations. Venture Philanthropy Partners, p.33-36. Retrieved from, http://www.vppartners.org/learning/reports/capacity/assessment.pdf

Mills, G. E. (2003). Action Research: A guide for the teacher researcher (2 ${ }^{\text {nd }}$ Edition). Merrill/Prentice-Hall: Upper saddle River, NJ.

Mills, G. E. (2011). Action Research: A guide for the teacher researcher (4 ${ }^{\text {th }}$ Edition). Pearson Education, Inc.: Upper saddle River, NJ.

Mills, C.W., (1959). The Sociological Imagination. New York, Oxford University Press.

Moore, C.W. (1996). The mediation process: Practical strategies for resolving conflict. ( $2^{\text {nd }}$ ed.). San Francisco: Jossey-Bass.

Morgan, D. (1997). Focus groups as qualitative research. Thousand Oaks, CA: Sage Publications.

Muhammad, A. (Ph.D.). (2009). Transforming school culture: How to overcome staff division. Forward by Richard DuFour. Bloomington, IN: Solution Tree Press.

National Association for the Education of Young Children. NAEYC, Washington, D.C. 
National Coalition Building Institute International (2009). 2008 Assessment Report for the Academic Success Center: "Understanding Community through Service and Coalition Building. ” Service Project Class (ALS 199). Retrieved from http://ncbi.org/wp-content/uploads/2009/04/als199-undercomm-thru-servcoalition409-final.pdf

O'Brien, R. (2001). Um exame da abordagem metodológica da pesquisa ação [An overview of the methodological approach of action research]. In Roberto Richardson (Ed.), Teoria e Prática da Pesquisa Ação [Theory and Practice of Action Research]. João Pessoa, Brazil: Universidade Federal da Paraíba. (English version). Retrieved from, http://www.web.net/ robrien/papers/xx\%20ar\%20final2.html

Paluck, E. L. \& Green, D. P. (2009). Prejudice reduction: What works? A review and assessment of research and practice. Annual Review of Psychology, 60, 339-367. DOI: 10.1146/annurev.psych.60.110707.163607

Pang, V.O. \& Park, C.D. (2003). Examination of the self-regulation mechanism: Prejudice reduction in pre-service teachers. Action Teach Education, 25(3).

Pate, G.S. (1995). Prejudice reduction and the findings of research. College of Education: University of Arizona.

Pettigrew, T. F., \& Tropp, L. R. (2000). Does intergroup contact reduce prejudice? Recent meta-analytic findings. In S. Oskamp (Ed.), Reducing prejudice and discrimination (pp. 93-114). Mahwah, NJ: Erlbaum.

Pettigrew, T. F., \& Tropp, L. R. (2006). A meta-analytic test of intergroup contact theory. Journal of Personality and Social Psychology, 90, 751-783. DOI: 10.1037/00223514.90.5.751.

Polizzi, J.A. (Ph.D.). (2007). Transformational Learning: The preparation of new teachers through experiential professional development. A thesis in educational leadership. (Unpublished doctoral dissertation). Pennsylvania State University.

Putnam, J., Gunnings-Moton, S. \& Sharp, C. (2009). Leadership through professional learning communities. Boston: McGraw-Hill Learning Solutions.

Purkey, W. W. (1992a). An introduction to invitational theory. Journal of Invitational Theory and Practice, 1, 5-15. Retrieved from http://www.invitationaleducation.net/journal/v11p5.htm.

Purkey, W. W. (1992b). Conflict resolution: An invitational approach. Retrieved from http://www.invitationaleducation.net/journal/v12p111.htm. 
Reed, C. (1996). Overcoming prejudices: An invitational approach. The Urban Review, 28(1), 81-93.

Rokeach, M. \& Rothman G. (1965). The principle of belief congruence and the congruity principle as models of cognitive interaction. Psychological Review, 72, 128-142.

Rubin, H. J. \& Rubin, I. S. (2005). Qualitative interviewing: The art of hearing data. $\left(2^{\text {nd }}\right.$ Ed.). Thousand Oaks, CA: Sage.

Rubin, M., Hewstone, M. \& Voci, A. (2001), Stretching the boundaries: Strategic perceptions of intragroup variability. European Journal of Social Psychology, 31: $413-429$.

Sherman, D. (2003). Critical theory. In R.C. Solomon \& D. Sherman (Eds.), The Blackwell guide to continental philosophy (pp. 188-218). Malden, MA: Blackwell.

Short, G. (1993). Prejudice reduction in schools: The value of inter-racial contact. British Journal of Sociology of Education, 14(2), 159 - 168.

Senge, P.M. (1990). The fifth discipline: The art and practice of the learning organization. New York, NY: Doubleday.

Senge, P.M (2000). Schools that learn. New York, NY: Doubleday.

Senge, P.M., Kleiner, A., Roberts, C., Ross, R.B. \& Smith, B.J. (1994). The fifth discipline fieldbook: Strategies and tools for building a learning organization. New York, NY: Doubleday.

Singh, B. R. (1991). Teaching methods for reducing prejudice and enhancing academic achievement for all children. Educational Studies, 17(2), 157 - 171.

Spiotta, G.E. (Ed.D.). (2004). Diversity and prejudice reduction action taken as a result of diversity leadership training for member high schools students: A compilation. (Unpublished doctoral dissertation). Seton Hall University.

Starling, G. (2002). Managing the public sector. $\left(6^{\text {th }}\right.$ Ed.). Forth Worth, TX: Thomas Learning Systems. Harcourt College Publishers.

Stephen, T. (2010). Successfully managing change in organizations. New York, NY: Industrial Press.

Stringer, E. T. (2007). Action research. Thousand Oaks, CA: Sage Publications.

Tatum, B.D. (Ph.D.). (1997). "Why are all the black kids sitting together in the cafeteria?” New York: Basic Books. 
Taylor, K. L. (2004). Through their eyes: A Strategic Response to the national achievement gap. Philadelphia, PA: Research for Better Schools.

Tidd, J. (2010). Managing innovation: Integrating technology, market and organizational change. New York, NY: Wiley.

Tillman, L. (2002). Culturally sensitive research approaches: An African-American perspective. Educational Researcher, 31(9), 3-12.

Titus, D. (1998). Teaching tolerance and appreciation for diversity: Applying the research on prejudice reduction. Paper presented at the $53^{\text {rd }}$ Annual Meeting of the Association for Supervision and Curriculum Development. San Antonio, TX. March 21-24, 1998.

Waltzer, M. (1987). Interpretation and social criticism. Cambridge, MA: Harvard University Press.

Wardle, F. (Ph.D.). (1999). Tomorrow's children: Meeting the needs of multiracial and multiethnic children, at home, in early childhood programs, and at school. Denver, CO: Center for the Study of Biracial Children.

Wardle, F. (Ph.D.). (2003a). Diversity workshop. Littleton, Colorado: Child Care Partnership February 17, 2003.

Wardle, F. (Ph.D.). (2003b). Introduction to early childhood education: A multidimensional approach to child-centered care and learning. Boston, MA: Allyn \& Bacon.

Wardle, F. (Ph. D.). (2007). Diversity in early childhood programs. Earlychildhood NEWS. The Professional Resource for Teachers and Parents. Retrieved from http://www.earlychildhoodnews.com/earlychildhood/article_view.aspx?ArticleID $=548$.

Watts, H. (1985). When teachers are researchers, teaching improves. Journal of Staff Development, 6 (2), 118-127.

Wells, G. (1994). Introduction: Teacher research and educational change. In G. Wells et al., Changing schools from within: Creating communities of Inquiry. Toronto: OISE Press.

Wiggins, G. \& McTighe, J. (2007). Schooling by design: Mission, action, and achievement. Alexandria, VA: Association for Supervision and Curriculum Development (ASCD). 
Willard-Holt, C. (2000). Preparing teachers for urban settings: Changing teacher education by changing ourselves. The Qualitative Report, 4(3/4). Retrieved from http://www.nova.edu/ssss/QR/QR4-3/willard.html

Wolcott, H.F. (1988). Ethnographic research in education . In R.M. Jaeger (Ed.), Complementary methods for research in education (pp. 187-210). Washington, DC: American Education Research Association.

Woodard, S.L. (1966). Belief congruence theory and school integration. Urban Education, 2 (3).

Wren, J.T. (Ed.). (1995). The leader's companion: Insights on leadership through the ages. New York, NY: Free Press. 


\section{APPENDIX A:}

\section{APPENDICES}

Letter Requesting Permission/Permission Granted

APPENDIX B:

Consent Forms - Informed Consent \& Consent for Survey

APPENDIX C:

IRB Application's Protocol Approval Letter

APPENDIX D:

Participant Pre Assessment Survey Responses Summary

APPENDIX E:

Participant Post Assessment Survey Responses Summary

APPENDIX F:

Participant Learning Reflection Survey

APPENDIX G:

Coding Document (central office administration interview protocol)

APPENDIX H:

Coding Document (site administration interview protocol)

APPENDIX I:

Coding Document (observation protocol data)

APPENDIX J:

Participant's Certificate of Completion

\section{APPENDIX K:}

Parallel Conversion Plan for Implementation Process

\section{APPENDIX L:}

Participant Six Month Follow Up Survey 


\section{APPENDIX A}

\section{LETTER REQUESTING PERMISSION/PERMISSION GRANTED}




\section{RE: REQUEST TO COMPLETE DOCTORAL RESEARCH STUDY (Ed.D. Dissertation Project)}

From : Michael Foster $<$ Michael.Foster@Phila.gov $>$

\section{Subject : RE: REQUEST TO COMPLETE DOCTORAL RESEARCH STUDY (Ed.D. Dissertation Project)}

To : bgordon816@comcast.net

Cc : Berdine Gordon <gordon19@students.rowan.edu >, Cheryl Randolph ×cherylran7@aol.com>, rcollier@bpumcdc.com

You have my permission to perform this study.

From: bgordon816@comcast.net [mailto:bgordon816@comcast.net]

Sent: Tuesday, March 15, 2011 12:04 AM

To: Michael Foster

Cc: Berdine Gordon; Cheryl Randolph; rcollier@bpumcdc.com

Subject: REQUEST TO COMPLETE DOCTORAL RESEARCH STUDY (Ed.D. Dissertation Project)

\section{Michael W. Foster, Board Chairman \\ BPUM Child Development Centers Inc. \\ 622 Cooper Street}

Camden, NJ 08102

\section{TO THE CHAIRMAN, BOARD OF DIRECTORS:}

Over 20 years ago, while attending Rutgers University as an undergraduate student, I completed one of the first comprehensive research papers on the History. Purpose, and Organizational Leadership structure of BPUM-EDC. The foundations of that initial compilation of written research work became a contributing archived document for the agency. Consequently, the information from the original written research work has developed 20 years later into an expanded dissertation topic on Educational Leadership. It is with this foundational work that I shall use my skilled and reflective learning practices to transform this knowledge into a scholarly written dissertation of Doctoral study.

I am a graduate student of the College of Professional and Continuing Education, Department of Educational Leadership at Rowan University. I am currently working on my doctoral dissertation project. After much reflection, the research question I wish to answer is:

How does leadership training skills on prejudice reduction result in changed practices in schools?

The subsidiary or analytic questions are:

1. What are the problems facing prejudice reduction practices in urban education?

2. What long-range effects of prejudice reduction practices have been proven positive to the efficacy of a diversity leadership framework?

3. What are the effects of practices on organizational attitudes and behavior outcomes of education?

4. What factors promote changes in reduction practice in schools? What factors impede changes in practice in schools?

5. What have been past responses in practice to diversity as a result of leadership training skills for schools on prejudice reduction? 


\section{APPENDIX B}

CONSENT FORMS - INFORMED CONSENT \& CONSENT FOR SURVEY 


\section{Informed Consent Form}

\section{Participants over the age of 18}

I agree to participate in a study entitled "The Impact of Diversity Leadership Training Skills on Prejudice Reduction for School Personnel," which is being conducted by Berdine Gordon-Littréan, a graduate Ed.D. student of the Educational Leadership Department, Rowan University.

The purpose of this study is to evaluate the methods used by individuals and groups to solve social justice issues involving diversity in educational leadership. The data collected in this exploratory study will be compiled and analyzed in order to make a report in considering future research study on informed practice and organizational change.

I understand that I will be required to attempt to solve a logic problem, and I will be assigned to work either individually or as part of a group training. My participation in the study should not exceed one hour for each training module completed. There are a total of eight (8) modules, and professional development training hours will be issued for my voluntary time and cooperation in the study.

I understand that my responses will be anonymous and that all the data gathered will be confidential. I agree that any information obtained from this study may be used in any way thought best for publication or education provided that I am in no way identified and my name is not used.

I understand that there are no physical or psychological risks involved in this study, and that I am free to withdraw my participation at any time without penalty.

I understand that my participation does not imply employment with the State of New Jersey, Rowan University, the principal investigator, or any other project facilitator.

If I have any questions or problems concerning my participation in this study, I may contact Berdine Gordon-Littréan, Doctor of Educational Leadership (Ed.D.) Student, at (856) 343-2811. Doctoral Committee Chairperson is: Dr. Yvonne E. González Rodríguez, and she may be contacted at (856) 256-4500 x3807.

(Signature of Participant)

(Date)

(Signature of Investigator)

(Date)

If you have any questions about your rights as a research subject, you may contact the Associate Provost for Research at: Rowan University Institutional Review Board for the Protection of Human Subjects, Office of Research, 201 Mullica Hill Road, Glassboro, NJ 08028-1701 • Tel: 856-256-5150 


\section{Consent for Survey}

I am conducting a study on The Impact of Diversity Leadership Training Skills for Schools on Prejudice Reduction. If you wish to participate in this study, please complete each survey according to the instructions given as directed. The surveys can be accessed via Survey Monkey to ensure anonymity and confidentiality of responses. Your participation in this study is voluntary. You may choose not to respond to any question or to not participate in the study as a whole with no penalty to you. If you have any questions, my name and contact information along with Doctoral Committee Chairperson's name and contact information appear below:

Berdine Gordon-Littréan, Principal Investigator (PI)

Doctor of Educational Leadership (Ed.D.) Student

gordon19@students.rowan.edu

(856) 343-2811

13 North Carlton Street

Lindenwold, NJ 08021

Dr. Yvonne E. González Rodríguez, Professor

Doctoral Committee Chairperson

Rodriguez@rowan.edu

(856) 256-4500 x3807

Rowan University

Department of Teacher Education

201 Mullica Hill Road

Glassboro, New Jersey 08028 
APPENDIX C

IRB APPLICATION'S PROTOCOL APPROVAL LETTER 


\section{Rowan University}

Septcombar $2 \$, 20.1$

Rordiue Dorilen-Liltreat:

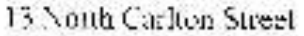

Lindenkeld, N.I 0\$O2I

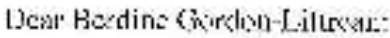

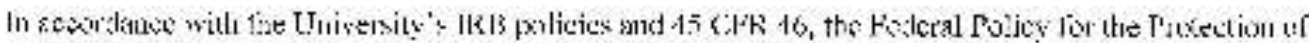

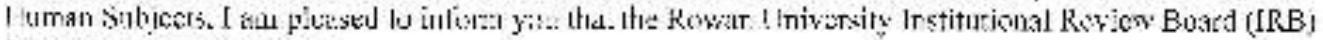
tas vimplod yeur projec:

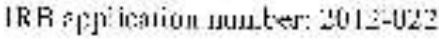

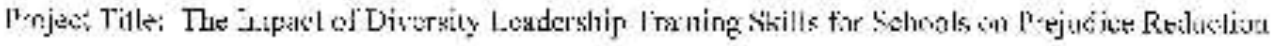

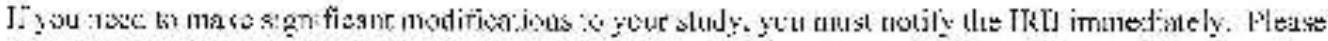

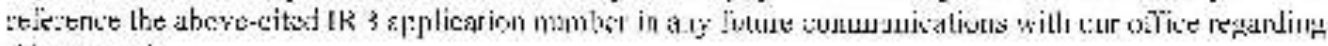
chis msint:its

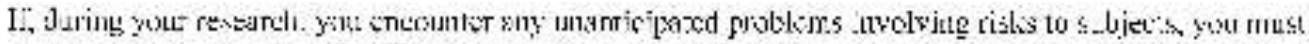

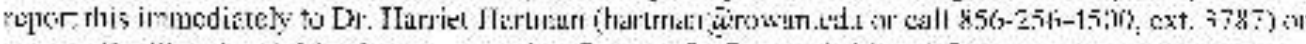

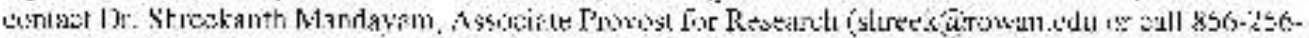
$5150 \%$

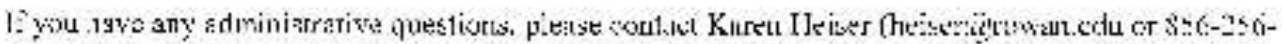
SLI0:

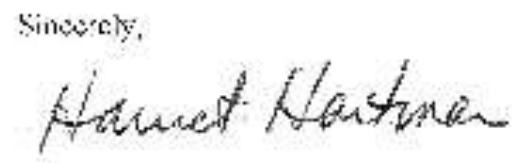

Hursier Jlerimen, H'l.LI.

Thar: Rostan Thivcosity IRF

c: Jame a Moce. Silser Ru. [kuzeulary Sc]uoj. Millville. XI 083$]$

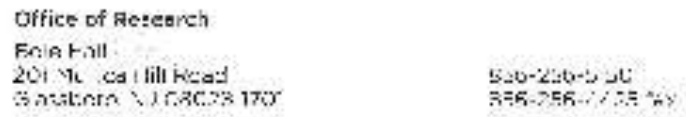


APPENDIX D

PARTICIPANT PRE ASSESSMENT SURVEY RESPONSES SUMMARY 


\section{Participant Pre Assessment Survey Responses Summary}

Survey instrument was adapted from National Coalition Building Institute (NCBI, 2009).

The Impact of Diversity Leadership Training Skills for Schools on Prejudice Reduction:

An exploratory study in Action Research

This appendix contains the actual Pre-training self assessment responses made by participants. The responses were taken from the survey created online, http://www.surveymonkey.com/. Research Question: How does diversity leadership training skills on prejudice reduction result in changed beliefs of discriminatory behavior in schools?

Total Collected Participant Survey Responses: n=14

1. I am at ease with people unlike myself.

5-Almost Always

4-Frequently

3-Sometimes

2-Rarely

1-Never
$35.7 \%$

$35.7 \%$

$21.4 \%$

$0.0 \%$

$7.1 \%$

2. I am able to identify the benefits of taking pride in the multiple components of who I am. (Examples: What is your Gender? Religion? Economic class? Ethnic heritage? Sexual orientation?)

5-Almost Always

4-Frequently

3-Sometimes

2-Rarely

1-Never
$64.3 \%$

$28.6 \%$

$7.1 \%$

$0.0 \%$

$0.0 \%$ 
3. I am uncomfortable when I hear a prejudicial slur or joke.

\begin{tabular}{|c|c|}
\hline 5-Almost Always & $21.4 \%$ \\
\hline 4-Frequently & $35.7 \%$ \\
\hline 3-Sometimes & $35.7 \%$ \\
\hline 2-Rarely & $0.0 \%$ \\
\hline 1-Never & $7.1 \%$ \\
\hline \multicolumn{2}{|c|}{ 4. I understand the impact of oppression and discrimination. } \\
\hline 5-Almost Always & $85.7 \%$ \\
\hline 4-Frequently & $14.3 \%$ \\
\hline 3-Sometimes & $0.0 \%$ \\
\hline 2-Rarely & $0.0 \%$ \\
\hline 1-Never & $0.0 \%$ \\
\hline \multicolumn{2}{|c|}{ 5. I am inclined to let people know that it bothers me when I hear prejudicial statement } \\
\hline 5-Almost Always & $35.7 \%$ \\
\hline 4-Frequently & $28.6 \%$ \\
\hline 3-Sometimes & $35.7 \%$ \\
\hline 2-Rarely & $0.0 \%$ \\
\hline 1-Never & $0.0 \%$ \\
\hline
\end{tabular}


6. I recognize my own prejudices.

\begin{tabular}{|c|c|}
\hline 5-Almost Always & $42.9 \%$ \\
\hline 4-Frequently & $42.9 \%$ \\
\hline 3-Sometimes & $14.3 \%$ \\
\hline 2-Rarely & $0.0 \%$ \\
\hline 1-Never & $0.0 \%$ \\
\hline \multicolumn{2}{|c|}{ 7. In most situations when discrimination is evident, I believe I can make a differenc } \\
\hline 5-Almost Always & $50.0 \%$ \\
\hline 4-Frequently & $28.6 \%$ \\
\hline 3-Sometimes & $21.4 \%$ \\
\hline 2-Rarely & $0.0 \%$ \\
\hline 1-Never & $0.0 \%$ \\
\hline \multicolumn{2}{|c|}{ 8. I feel comfortable letting others know who I am-- even if I am "different." } \\
\hline 5-Almost Always & $71.4 \%$ \\
\hline 4-Frequently & $0.0 \%$ \\
\hline 3-Sometimes & $21.4 \%$ \\
\hline 2-Rarely & $0.0 \%$ \\
\hline 1-Never & $7.1 \%$ \\
\hline
\end{tabular}


9. I trust people will fully accept me as ME!

\begin{tabular}{|lr|}
\hline 5-Almost Always & $21.4 \%$ \\
\hline 4-Frequently & $\mathbf{5 0 . 0 \%}$ \\
\hline 3-Sometimes & $14.3 \%$ \\
\hline 2-Rarely & $14.3 \%$ \\
\hline 1-Never & $0.0 \%$ \\
\hline
\end{tabular}

10. I am personally committed to work toward eliminating discrimination and mistreatment of oppressed groups.

\begin{tabular}{|lc|}
\hline 5-Almost Always & $\mathbf{7 8 . 6 \%}$ \\
\hline 4-Frequently & $14.3 \%$ \\
\hline 3-Sometimes & $7.1 \%$ \\
\hline 2-Rarely & $0.0 \%$ \\
\hline 1-Never & $0.0 \%$ \\
\hline
\end{tabular}




\section{APPENDIX E}

PARTICIPANT POST ASSESSMENT SURVEY RESPONSES SUMMARY 


\section{Participant Post Assessment Survey Responses Summary}

Survey instrument was adapted from National Coalition Building Institute (NCBI, 2009).

The Impact of Diversity Leadership Training Skills for Schools on Prejudice Reduction:

An exploratory study in Action Research

This appendix contains the actual Post-training self assessment responses made by participants. The responses were taken from the survey created online, http://www.surveymonkey.com/. Research Question: How does diversity leadership training skills on prejudice reduction result in changed beliefs of discriminatory behavior in schools?

Total Collected Participant Survey Responses: n=14

1. I am at ease with people unlike myself.

5-Almost Always

4-Frequently

3-Sometimes

2-Rarely

1-Never
$64.3 \%$

$14.3 \%$

$14.3 \%$

$7.1 \%$

$0.0 \%$

2. I am able to identify the benefits of taking pride in the multiple components of who I am. (Examples: What is your Gender? Religion? Economic class? Ethnic heritage? Sexual orientation?)

5-Almost Always

4-Frequently

3-Sometimes

2-Rarely

1-Never
$71.4 \%$

$28.6 \%$

$0.0 \%$

$0.0 \%$

$0.0 \%$ 
3. I am uncomfortable when I hear a prejudicial slur or joke.

\begin{tabular}{|c|c|}
\hline 5-Almost Always & $50.0 \%$ \\
\hline 4-Frequently & $14.3 \%$ \\
\hline 3-Sometimes & $21.4 \%$ \\
\hline 2-Rarely & $14.3 \%$ \\
\hline 1-Never & $0.0 \%$ \\
\hline \multicolumn{2}{|c|}{ 4. I understand the impact of oppression and discrimination. } \\
\hline 5-Almost Always & $85.7 \%$ \\
\hline 4-Frequently & $14.3 \%$ \\
\hline 3-Sometimes & $0.0 \%$ \\
\hline 2-Rarely & $0.0 \%$ \\
\hline 1-Never & $0.0 \%$ \\
\hline \multicolumn{2}{|c|}{ 5. I am inclined to let people know that it bothers me when I hear prejudicial statement } \\
\hline 5-Almost Always & $57.1 \%$ \\
\hline 4-Frequently & $14.3 \%$ \\
\hline 3-Sometimes & $28.6 \%$ \\
\hline 2-Rarely & $0.0 \%$ \\
\hline 1-Never & $0.0 \%$ \\
\hline
\end{tabular}


6. I recognize my own prejudices.

\begin{tabular}{|c|c|}
\hline 5-Almost Always & $64.3 \%$ \\
\hline 4-Frequently & $28.6 \%$ \\
\hline 3-Sometimes & $7.1 \%$ \\
\hline 2-Rarely & $0.0 \%$ \\
\hline 1-Never & $0.0 \%$ \\
\hline \multicolumn{2}{|c|}{ 7. In most situations when discrimination is evident, I believe I can make a differenc } \\
\hline 5-Almost Always & $57.1 \%$ \\
\hline 4-Frequently & $28.6 \%$ \\
\hline 3-Sometimes & $14.3 \%$ \\
\hline 2-Rarely & $0.0 \%$ \\
\hline 1-Never & $0.0 \%$ \\
\hline \multicolumn{2}{|c|}{ 8. I feel comfortable letting others know who I am-- even if I am "different." } \\
\hline 5-Almost Always & $64.3 \%$ \\
\hline 4-Frequently & $21.4 \%$ \\
\hline 3-Sometimes & $7.1 \%$ \\
\hline 2-Rarely & $7.1 \%$ \\
\hline 1-Never & $0.0 \%$ \\
\hline
\end{tabular}


9. I trust people will fully accept me as ME!

5-Almost Always

$42.9 \%$

4-Frequently

$35.7 \%$

3-Sometimes

$7.1 \%$

2-Rarely

$14.3 \%$

1-Never

$0.0 \%$

10. I am personally committed to work toward eliminating discrimination and mistreatment of oppressed groups.

5-Almost Always

$76.9 \%$

4-Frequently

$23.1 \%$

3-Sometimes

$0.0 \%$

2-Rarely

$0.0 \%$

1-Never

$0.0 \%$ 
APPENDIX F

PARTICIPANT LEARNING REFLECTION SURVEY 


\section{Participant Learning Reflection Survey \\ (What I learned is...The most useful part of this skills training experience was...)}

The Impact of Diversity Leadership Training Skills for Schools on Prejudice Reduction:

An exploratory study in Action Research

This appendix contains the actual shared learning reflections survey tool used voluntarily by participants. The sample reflections completed and obtained were taken from a secured data source created online, http://www.surveymonkey.com/.

INSTRUCTIONS: Please provide your feedback on what you have learned.

Survey instrument constructed by the researcher along with reflective adaptations from National Coalition Building Institute (NCBI, 2009).

*1. What new information did you learn?

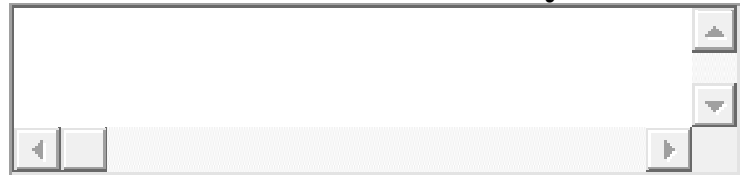

*2. What was the most useful part of this skills training experience?

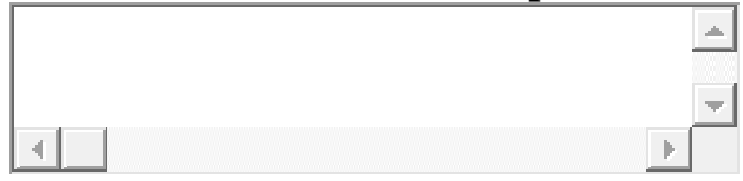

*3. Do you have any suggestions for improvement in the delivery of training information, specifically on informed practice?

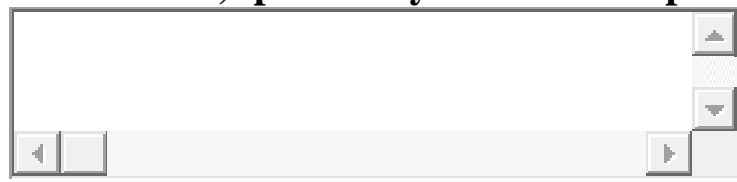

*4. What new insights did you learn about yourself?

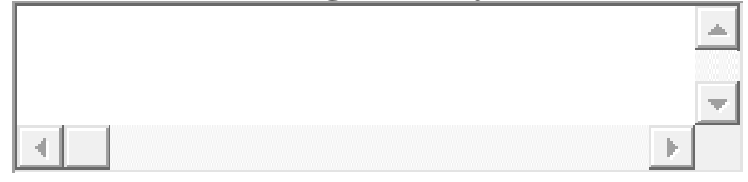

*5. What new insights did you learn about others?

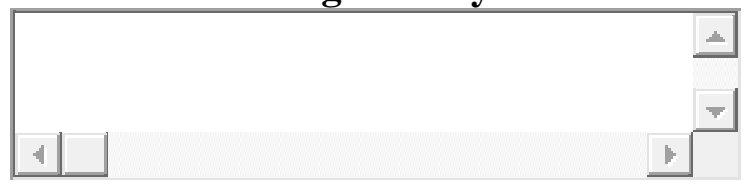


*6. What new skills did you learn?

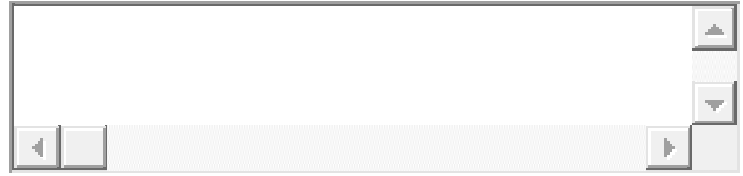

*7. What were some things provided from the trainer's resource information that were particularly helpful or meaningful to you?

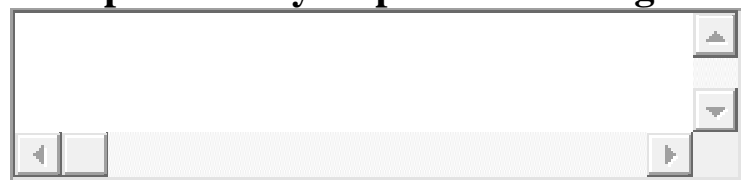

*8. Give one example of how you will use your newly learned Diversity skills in your life/workplace/etc.

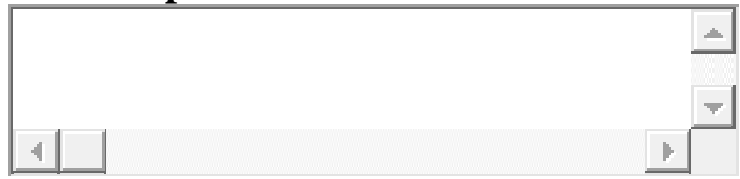

*9. Is there anything you will do differently now to create a welcoming and safe environment for all groups in your school program, community, and/or business workplace? Please explain your "Y or N" responses.

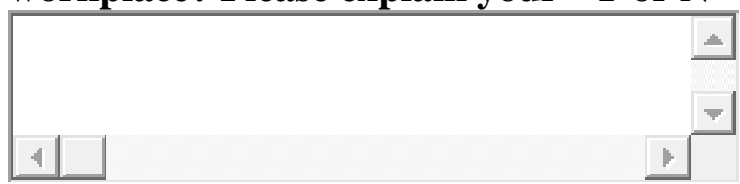

10. How do you rate overall significance and importance on the measured impact of this diversity leadership skills training?

5-Essential

4-Very Important

3-Somewhat Important

C 2-Neither Important or Not Important

C 1-Not Important

\section{Done}

Powered by SurveyMonkey

Check out our sample surveys and create your own now! 


\section{APPENDIX G \\ CODING DOCUMENT \\ CENTRAL OFFICE ADMINISTRATION INTERVIEW PROTOCOL INTERVIEW RESPONSES SUMMARY}




\section{Coding Document for Central Office Administration Interview Protocol Interview Responses Summary}

The Impact of Diversity Leadership Training Skills for Schools on Prejudice Reduction: An exploratory study in Action Research

This appendix contains the actual coding scheme to agency interview protocol transcripts from Board Member/Central Office Program Administration participants.

\begin{tabular}{|c|c|}
\hline \multicolumn{2}{|r|}{ Diversity } \\
\hline $\begin{array}{l}\text { Coding } \\
\text { Category/Label }\end{array}$ & Example from Data Collection/Criteria (Interview Protocol) \\
\hline $\begin{array}{r}\mathbf{Q}_{1} \\
\text { Vision }\end{array}$ & $\begin{array}{l}\text { B - Equity by law and enforced across all genders, races and } \\
\text { classes } \\
\mathrm{A}^{1}-\mathrm{A} \text { person is able to respond to anyone diverse background } \\
\text { using reflective listening skills. } \\
\mathrm{A}^{2}-\text { An assorted amount of people and ideas }\end{array}$ \\
\hline $\begin{array}{r}\mathbf{Q}_{2} \\
\text { Communication (Vision } \\
\text { for Student Learning) }\end{array}$ & $\begin{array}{l}\mathrm{B}-\text { Review } 10 \text { scientific facts about race and } 10 \text { problems with } \\
\text { race and a discussion of white privilege nationally and globally. } \\
\mathrm{A}^{1}-\text { As educators we must make a connection to every student } \\
\text { within our school community. } \\
\mathrm{A}^{2}-\text { Through visual mechanisms }\end{array}$ \\
\hline $\begin{array}{r}\mathbf{Q}_{3} \\
\text { Communication (Center } \\
\text { Locations' Vision) }\end{array}$ & $\begin{array}{l}\text { B - Based on the information shared and accepted by them to } \\
\text { participate in educational programming } \\
A^{1}-\text { Role Modeling } \\
A^{2}-\text { [The respondent was "Unsure" on how to answer. Proceed to } \\
\text { next question.] }\end{array}$ \\
\hline $\begin{array}{r}\mathbf{Q}_{4} \\
\text { Communication (Vision } \\
\text { Alignment to Agency } \\
\text { Mission) }\end{array}$ & $\begin{array}{l}\mathrm{B}-\text { Seen through daily exercises, role play and life experiences } \\
\text { reviewed with students observed in Board "walk-through" } \\
\mathrm{A}^{1} \text { - The vision is communicated by the actions of leadership. } \\
\mathrm{A}^{2}-\text { [The respondent was "Unsure" on how to answer. Proceed to } \\
\text { next question.] }\end{array}$ \\
\hline $\mathbf{Q}_{5}$ & B-Yes, to a proactive diversity program for which goals can be \\
\hline
\end{tabular}




\begin{tabular}{|c|c|}
\hline $\begin{array}{r}\text { Change in Instructional } \\
\text { Practice (Implemented } \\
\text { diversity/reform } \\
\text { initiative) }\end{array}$ & $\begin{array}{l}\text { measured and quantified } \\
\mathbf{A}^{1}-\text { I have always put all students first. } \\
\mathbf{A}^{2}-\text { N/A. }\end{array}$ \\
\hline $\begin{array}{r}\mathbf{Q}_{6} \\
\text { Supervisory Evaluation } \\
\text { Procedures/Assessment } \\
\text { on Change }\end{array}$ & $\begin{array}{l}\text { B -No, we have been using the same procedures for } 6 \text { years. } \\
A^{1}-N O \text {, evaluation procedures have not changed. } \\
A^{2} \text {-N/A. }\end{array}$ \\
\hline \begin{tabular}{r|}
$\mathbf{Q}_{7}$ \\
Center Locations \\
Feedback on Changes
\end{tabular} & $\begin{array}{l}\text { B -We have to seek to create an effective environment by making } \\
\text { sure all staff have accepted and implemented [this] approach to } \\
\text { diversity and have it reflected in the curriculum and conduct of } \\
\text { everyday interactions among staff, students, parents, and visitors. } \\
\mathrm{A}^{1}-\text { The mandates from the Department of Education has made } \\
\text { school districts more aware in regards to our BOE population and } \\
\text { regular preschool population. } \\
\mathrm{A}^{2} \text {-N/A. }\end{array}$ \\
\hline $\begin{array}{r}\text { Q8 } \\
\text { Mission Impact (Multi- } \\
\text { cultural Education) }\end{array}$ & $\begin{array}{l}\text { B -Provided a more educationally pleasing and supportive } \\
\text { learning environment. } \\
\mathrm{A}^{1} \text {-Legislation has made an influence on the delivery of } \\
\text { instructional curriculum in alignment to mission. } \\
\mathrm{A}^{2}-\mathrm{N} / \mathrm{A} \text {. }\end{array}$ \\
\hline \begin{tabular}{r|} 
Q9 \\
Professional \\
Development \& \\
Accountability
\end{tabular} & $\begin{array}{l}\text { B -Workshops and hands-on training are essential in } \\
\text { understanding the reality of the diverse world we lived in today. } \\
\text { The need for social skills, which emphasizes equality of results } \\
\text { when interacting with a diversity of people. } \\
\mathrm{A}^{1} \text { - Administrators, teachers, student, parents and non- } \\
\text { instructional need to be provided with the proper professional } \\
\text { development programs and be instructed by experts in the field. } \\
\mathrm{A}^{2} \text {-Allocating funds to attend conferences, and return to share } \\
\text { learned experiences in our in-services. }\end{array}$ \\
\hline $\begin{array}{r}Q_{10} \\
\text { Resources (Accomplish } \\
\text { PD Goals) }\end{array}$ & $\begin{array}{l}\text { B - Serious review (literature) of the scientific basis of race and } \\
\text { frank discussions in workshops; } A^{1}-\text { Funding support } \\
A^{2}-\text { Benefit from additional resources if available. }\end{array}$ \\
\hline
\end{tabular}




\author{
APPENDIX H \\ CODING DOCUMENT \\ SITE ADMINISTRATION INTERVIEW PROTOCOL \\ INTERVIEW RESPONSES SUMMARY
}




\section{Coding Document for Site Administration Interview Protocol Interview Responses Summary}

The Impact of Diversity Leadership Training Skills for Schools on Prejudice Reduction: An exploratory study in Action Research

This appendix contains the actual coding scheme to agency interview protocol transcripts from the Site Supervisor/Center Director participants.

\begin{tabular}{|c|c|}
\hline \multicolumn{2}{|r|}{ Diversity } \\
\hline $\begin{array}{l}\text { Coding } \\
\text { Category/Label }\end{array}$ & Example from Data Collection/Criteria (Interview Protocol) \\
\hline Vision & $\begin{array}{l}C^{1}-\text { All children are treated equally. } \\
C^{2} \text {-Everyone to be treated with respect and fairness } \\
C^{3} \text {-My vision is that educators and service providers treat } \\
\text { students/ families with respect and dignity. } \\
C^{4}-\text { To make sure that the accomplishments of all groups are } \\
\text { properly displayed }\end{array}$ \\
\hline $\begin{array}{r}\mathbf{Q}_{2} \\
\\
\text { Communication } \\
\text { (Directors/Supervisors' } \\
\text { Vision for Student } \\
\text { Learning) }\end{array}$ & $\begin{array}{l}C^{1}-\text { What is expected for one is expected for all. } \\
C^{2} \text {-Developmentally appropriate activities } \\
C^{3} \text {-By embracing and accepting students and families where they } \\
\text { are. Try to understand and learn about their experiences to meet } \\
\text { their needs. } \\
C^{4}-\text { The social interaction and their exposure to children of } \\
\text { different cultures }\end{array}$ \\
\hline $\begin{array}{r}\text { Communication } \\
\text { (Head/Lead/Group } \\
\text { Teachers' Vision) }\end{array}$ & $\begin{array}{l}C^{1}-\text { Professional Development and Role Modeling. } \\
C^{2} \text {-Implementing in the lesson plan } \\
C^{3} \text {-In her classroom mission statement and letter to families. The } \\
\text { way in which the classroom environment is set up. } \\
C^{4}-\text { Through field trips to museums throughout the year }\end{array}$ \\
\hline
\end{tabular}




\begin{tabular}{|c|c|}
\hline $\begin{array}{r}\mathbf{Q}_{4} \\
\text { Communication } \\
\text { (Teachers' Vision } \\
\text { Alignment to Center's } \\
\text { Mission) }\end{array}$ & $\begin{array}{l}\mathrm{C}^{1} \text {-Diversity is encouraged by the } \mathrm{BOE} \text {. } \\
\mathrm{C}^{2} \text {-Parent and employee handbook } \\
\mathrm{C}^{3}-\mathrm{By} \text { the program offered and the materials that are available } \\
\text { and distributed in their home language, etc. } \\
\mathrm{C}^{4} \text {-[The respondent was "Unsure" on how to answer. Proceed to next } \\
\text { question.] }\end{array}$ \\
\hline $\begin{array}{r}Q_{5} \\
\text { Change in Instructional } \\
\text { Practice (Implemented } \\
\text { diversity/reform } \\
\text { initiative) }\end{array}$ & $\begin{array}{l}\mathrm{C}^{1}-\mathrm{No} \\
\mathrm{C}^{2}-\mathrm{No} \\
\mathrm{C}^{3}-\mathrm{No} \\
\mathrm{C}^{4}-\mathrm{No}\end{array}$ \\
\hline $\begin{array}{r}\mathbf{Q}_{6} \\
\text { Supervisory Evaluation } \\
\text { Procedures/Assessment } \\
\text { on Change }\end{array}$ & $\begin{array}{l}C^{1}-\text { No. } \\
C^{2}-\text { No. } \\
C^{3}-\text { No. [However], ensuring that materials are available, } \\
\text { accessible and displayed for the children. } \\
C^{4}-\text { No. }\end{array}$ \\
\hline $\begin{array}{r}Q_{7} \\
\text { Center Locations } \\
\text { Feedback on Changes }\end{array}$ & $\begin{array}{l}C^{1}-[\text { The respondent was "Unsure" on how to answer. Proceed to } \\
\text { next question.] } \\
C^{2} \text {-Yes. Anticipate positive changes if implementing diversity } \\
\text { initiative. } \\
C^{3} \text {-The environment improvements have been embraced. } \\
C^{4} \text {-Reaction only to reorganization of agency changes. }\end{array}$ \\
\hline $\begin{array}{r}Q_{8} \\
\text { Mission Impact (Multi- } \\
\text { cultural Education) }\end{array}$ & $\begin{array}{l}C^{1} \text {-The multicultural initiative is important considering the } \\
\text { diverse neighborhoods we serve at present. } \\
C^{2} \text {-Creates an awareness } \\
C^{3} \text {-It has increased awareness about the lack of diversity } \\
\text { materials available and accessible to all children and families. } \\
C^{4}-A \text { program has not been implemented but will benefit } \\
\text { program if we are to move forward. }\end{array}$ \\
\hline Q9 & $\mathrm{C}^{1}-$ More access to current reading materials \\
\hline
\end{tabular}




\begin{tabular}{|r|l|}
\hline $\begin{array}{r}\text { Professional } \\
\text { Development (Support) } \\
\text { \& Accountability }\end{array}$ & $\begin{array}{l}\mathrm{C}^{2} \text {-Monthly meetings } \\
\text { as the Thomas Edison State College English Language Learners } \\
\text { Summer Institute. }\end{array}$ \\
& $\begin{array}{l}\mathrm{C}^{4} \text {-Attending seminars or hiring consultants to give presentations } \\
\text { should facilitate developmental activities. }\end{array}$ \\
\hline $\mathbf{Q}_{10}$ & $\mathrm{C}^{1}$-Financial Resources \\
$\begin{array}{r}\text { Resources (Accomplish } \\
\text { Center PD Goals) }\end{array}$ & $\mathrm{C}^{2}$-Handouts (media and resource materials) \\
& $\mathrm{C}^{3}$-Additional funding for professional development relief time. \\
& $\mathrm{C}^{4}$-Proper funding and a commitment from staff. \\
\hline
\end{tabular}


APPENDIX I

CODING DOCUMENT

PARTICIPANT OBSERVATIONAL PROTOCOL

DIVERSITY LEADERSHIP SKILLS AND SCHOOL APPLICATION 


\section{Coding Document for Participant Observational Protocol Diversity Leadership Skills and School Application}

The Impact of Diversity Leadership Training Skills for Schools on Prejudice Reduction:

An exploratory study in Action Research

This appendix contains the actual coding scheme to categorize educator comments about diversity leadership skills and school application from the eight week professional development training. The categories were based on examples collected from observation protocol data use with the Prejudice Reduction Workshop Model's eight week module activities. Some of the coding references are expressed in a word or short phrase while other coding references are expressed in one or more phrases.

\begin{tabular}{|l|l|}
\hline \multicolumn{2}{|c|}{ Diversity Leadership Skills } \\
\hline $\begin{array}{l}\text { Coding } \\
\text { Category/Label }\end{array}$ & Example from Data Collection/Criteria (Observation Protocol) \\
\hline $\begin{array}{l}\text { Workshop \#1: } \\
\text { Leadels of }\end{array}$ & $\begin{array}{l}\text { Refers to knowing and practicing: Code Awareness } \\
\text { Self Awareness - examine self } \\
\text { - Other Awareness - being an ally to others }\end{array}$ \\
\hline $\begin{array}{l}\text { Wnvironmental } \\
\text { Scanning }\end{array}$ & $\begin{array}{l}\text { Refers to respecting and welcoming diversity in all forms - "Every } \\
\text { Group Counts." }\end{array}$ \\
\hline $\begin{array}{l}\text { Code Asking: "Did we leave anybody out?"; Code Behavior and } \\
\text { Conduct }\end{array}$ \\
$\begin{array}{l}\text { Stereotyping: 1 } \\
\text { Thoughts }\end{array}$ & $\begin{array}{l}\text { Refers to understanding how stereotypes impact our actions and } \\
\text { attitudes and how we can change them. } \\
\text { Code Values and Beliefs }\end{array}$ \\
\hline $\begin{array}{l}\text { Workshop \#4: } \\
\text { Internalized } \\
\text { Oppression }\end{array}$ & $\begin{array}{l}\text { Refers to learning the negative impact that stereotypes have on our } \\
\text { own and different groups' self image and behavior. } \\
\text { Code Espoused (Disposition) }\end{array}$ \\
\hline
\end{tabular}




\section{Coding Document for Participant Observational Protocol Diversity Leadership Skills and School Application}

\begin{tabular}{|l|l|}
\hline \multicolumn{2}{|c|}{ Diversity Leadership Skills } \\
\hline Coding & Example from Data Collection/Criteria (Observation Protocol) \\
\hline Workshop \#5: & $\begin{array}{l}\text { Refers to encouraging the expression of pride in all groups and } \\
\text { understanding the difference between authentic pride and } \\
\text { chauvinism. Code Motivation \& Capacity Building } \\
\text { Great To Be" }\end{array}$ \\
\hline Workshop \#6: & $\begin{array}{l}\text { Educators gain a new perspective on awareness and information } \\
\text { on how different groups experience mistreatment and learning } \\
\text { how to be better allies to them. } \\
\text { Becoming Allies }\end{array}$ \\
\hline $\begin{array}{l}\text { Code Competencies (Theory-in-use) } \\
\text { Making } \\
\text { Commitments to } \\
\text { Change }\end{array}$ & $\begin{array}{l}\text { Educators exercise listening skills in learning how different types } \\
\text { of discrimination affect individuals and groups. } \\
\text { Code Learn the power of the personal story and how it can effect } \\
\text { change. }\end{array}$ \\
\hline $\begin{array}{l}\text { Workshop \#8: } \\
\text { Shifting Attitudes }\end{array}$ & $\begin{array}{l}\text { Refers to reflective learning practicing in knowing the 3 Levels of } \\
\text { Leadership as an ally for preventing and interrupting prejudicial } \\
\text { jokes, comments, and slurs. Noticing and supporting both the } \\
\text { perpetrator and victim of prejudicial jokes, comments and slurs. } \\
\text { Code Learning by Doing (Explicit knowledge into Tacit } \\
\text { knowledge) }\end{array}$ \\
\hline
\end{tabular}




\section{Coding Document for Participant Observational Protocol Diversity Leadership Skills and School Application}

\begin{tabular}{|c|c|}
\hline \multicolumn{2}{|r|}{ School Application } \\
\hline $\begin{array}{l}\text { Coding } \\
\text { Category/Label }\end{array}$ & Example from Data Collection/Criteria (Observation Protocol) \\
\hline $\begin{array}{l}\text { Workshop \#1: } \\
\text { School Community } \\
\text { as Peers (including } \\
\text { student, faculty, and } \\
\text { staff leaders) }\end{array}$ & $\begin{array}{l}\text { Refers to recognizing that in whatever roles we have within the } \\
\text { school, we can take different levels of leadership to enhance the } \\
\text { school environment for ourselves and others. } \\
\text { Code Organizational Culture and EQ (Emotional Intelligence) }\end{array}$ \\
\hline $\begin{array}{l}\text { Workshop \#2: } \\
\text { Ups/Downs Activity }\end{array}$ & $\begin{array}{l}\text { Educators learn how to notice which school groups get respect and } \\
\text { which groups get excluded and reaching out to them. } \\
\text { Code Abilities (Human Capacities) }\end{array}$ \\
\hline $\begin{array}{l}\text { Workshop \#3: } \\
\text { First Thoughts } \\
\text { Exercise }\end{array}$ & $\begin{array}{l}\text { Educators learn through these demonstrated exercises how to } \\
\text { effectively examine the stereotypes within the school that create } \\
\text { tension and conflict. } \\
\text { Code Learning by Doing (Explicit knowledge into Tacit } \\
\text { knowledge); Espoused (Disposition) }\end{array}$ \\
\hline $\begin{array}{l}\text { Workshop \#4: } \\
\text { Controversial Issues } \\
\text { Process }\end{array}$ & $\begin{array}{l}\text { Refers to learned conflict resolution skills for handling and } \\
\text { resolving tough interpersonal/intergroup conflicts. } \\
\text { Code Exploring ways to "contradict" and to interrupt the } \\
\text { internalization of negative stereotypes on all groups and reduce } \\
\text { behaviors towards them. }\end{array}$ \\
\hline
\end{tabular}




\section{Coding Document for Participant Observational Protocol Diversity Leadership Skills and School Application}

\begin{tabular}{|c|c|}
\hline \multicolumn{2}{|r|}{ School Application } \\
\hline $\begin{array}{l}\text { Coding } \\
\text { Category/Label }\end{array}$ & Example from Data Collection/Criteria (Observation Protocol) \\
\hline $\begin{array}{l}\text { Workshop \#5: } \\
\text { Building Capacity \& } \\
\text { Authentic Cross- } \\
\text { Cultural } \\
\text { Relationships }\end{array}$ & $\begin{array}{l}\text { Creating opportunities for students, staff, and all groups to express } \\
\text { pride and receive recognition. } \\
\text { Code Motivation and Engagement }\end{array}$ \\
\hline $\begin{array}{l}\text { Workshop \#6: } \\
\text { Caucus Groups }\end{array}$ & $\begin{array}{l}\text { Identifying school groups where there is mistreatment and } \\
\text { misinformation and correcting it. } \\
\text { Code Behavior and Conduct; Skills (Knowledge In-action) }\end{array}$ \\
\hline $\begin{array}{l}\text { Workshop \#7: } \\
\text { Speak Outs }\end{array}$ & $\begin{array}{l}\text { Refers specifically to capacity building through group work and } \\
\text { determining the Speak Outs that need to be told and heard in order } \\
\text { to make the school environment safe and welcoming for all. } \\
\text { Code Engagement }\end{array}$ \\
\hline $\begin{array}{l}\text { Workshop \#8: } \\
\text { Role Playing and } \\
\text { Skits }\end{array}$ & $\begin{array}{l}\text { Refers to demonstration and application in identifying the most } \\
\text { common situations in school where individuals experience teasing } \\
\text { and put-downs and taking action to interrupt the behavior and/or } \\
\text { support the victims. } \\
\text { Code Understanding and Skills (Knowledge In-action) }\end{array}$ \\
\hline
\end{tabular}


APPENDIX J

CERTIFICATE OF COMPLETION 
Certificate of Completion

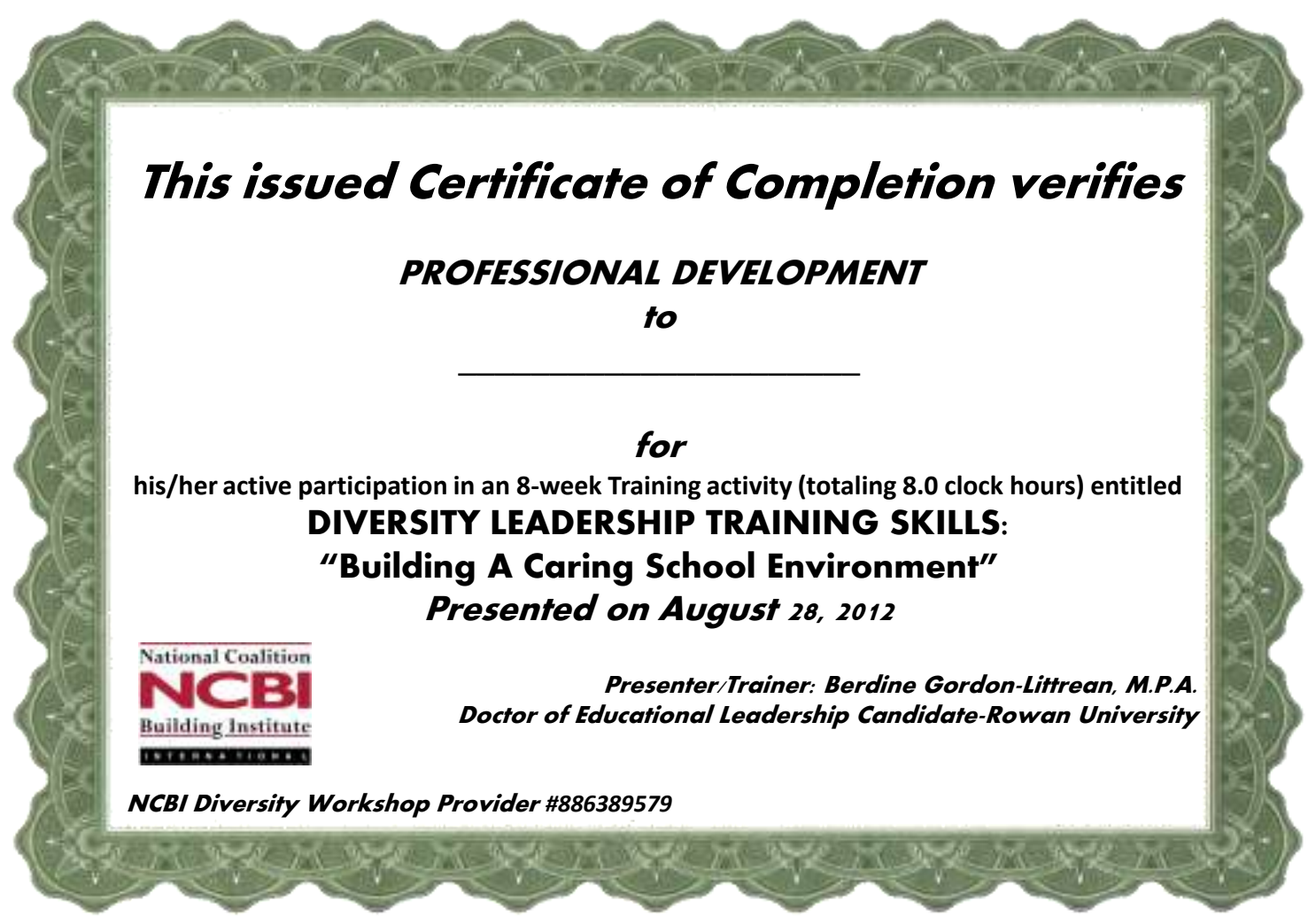




\section{APPENDIX K}

PARALLEL CONVERSION PLAN FOR IMPLEMENTATION PROCESS 


\section{Parallel Conversion Plan for Implementation Process}

This appendix contains a parallel conversion plan as suggestive (for consulting purposes) to adapt in monitoring implementation for articulating the connections between new behaviors and organizational success. This plan is the use of both systems simultaneously to ensure the new system works correctly. As staff is trained and phases are successfully evaluated old system will be shut down. Framework adapted from FirstClickSEO, LLC. (2007). Conversions. Retrieved from, http://www.firstclickseo.com/services/seoprocess.html

MONTH 1. Program Set up and Initial School Improvement Plan Optimization:

- Revise mission in alignment to improvement plan for content/clarity

- Implement initial change initiative optimization

- Integrate new School Improvement Plan processes into learning communities; operating simultaneously with legacy pedagogy.

\section{MONTHS 2-5. Focus on Program Performance of School Improvement Plan} (Asynchronous/Synchronous)

- Examination of Logical and Physical Data Flow patterns

- Review of the prejudice reduction modules usage patterns

- Review of key phrase logical and physical data flow by output

Success factors: teaching and learning practices; pass gains according to previous evaluations of mission, professional development inservices and input of the administrative instructional team leads.

Outcomes: assessment data (annual program audit) and use results to make plans for improving sub-group student performance; improvements in continuum of intervention services and prevention-oriented strategies.

Summative assessments: Annual Reviews

Limitations: based on assessed improvement areas for immediate action plan measures (to ensure that there are no detrimental effects or barriers of the mission process through the evaluation of the administrative instructional leads)

口 Synchronous (formative assessment)

- Development of reference point for conversion tracking of behavior reduction practices

MONTHS 6-9. Focus on Data Flows/Users based on newly aligned entity structures

- Focus on keyword mission in alignment to School Improvement Plan's priority area focus goals, objectives, and performance target 
indicators. Begin development on new key phrase rich Training and Assessment content

MONTHS 10-12. Focus on Conversions: Increase conversion percentages of entity usage for new change initiative practices for overall system evaluation, planning and analysis processes development in the next phase conversion with Training and Assessment system. 
APPENDIX L

PARTICIPANT SIX MONTH FOLLOW UP SURVEY 


\section{Participant Six Month Follow Up Survey Diversity Leadership Training Skills Application}

Survey instrument was designed and constructed by the researcher.

The Impact of Diversity Leadership Training Skills on Prejudice Reduction for School Personnel:

An exploratory study in Action Research

This appendix contains the actual six month post training self assessment survey used by participants. Three survey questions were administered: Two of the questions, participants were given the option to select multiple choices from the survey's multiple answers. The third question was a Likert type scale to measure likelihood (5=To a Great Extent, $4=$ To a Considerable Degree, $3=$ Somewhat, $2=$ Very Little, and 1=Not at All). The individual responses were taken from the survey created online, http://www.surveymonkey.com/.

Total Collected Participant Survey Responses: $\mathrm{n}=10$

\section{Diversity Leadership Training Skills Application}

Name of Presenter/Trainer: Berdine Gordon-Littréan, M.P.A. (Ed.D. Candidate)

The researcher is conducting a brief follow-up 6 month evaluation of the 8-week

Diversity Leadership Skills module training you attended last summer from July 2012 to August 2012. Your participation is voluntary and responses are completely confidential. The researcher will use unique identification codes to link your answers to information collected during the time of the 8-week training. This code system will not be used to identify you by name or obtain other information about you.

\footnotetext{
*1. Which module(s) skills have you found useful in your work since you came back from training:

Three Levels of Leadership

$\ulcorner$ Environmental Scanning: Ups/Downs Activity

$\ulcorner$ Stereotyping: 1st Thoughts Exercise

$\ulcorner$ Internalized Oppression

$\ulcorner$ Building Pride/Capacity Building

$\ulcorner$ Becoming Allies: Caucus Groups Activity

$\ulcorner$ Making Commitments to Change: Speak Outs

$\ulcorner$ Shifting Attitudes: Role Playing and Skits
} 


\section{*2. Which areas of skills application have you shared with colleagues?}

Recognizing that in whatever roles we have within the school, we can take different levels of leadership to enhance the school environment for ourselves and others.

Help to create an environment that values and welcomes diversity; reach out to groups or individuals (Asking: "Did we leave anybody out?").

Be willing to be aware of and re-examine our records about different groups and individuals that create tension and conflict and then get new information.

Examine our own I.O. attitudes and behaviors in our school community. Make changes where needed by exploring ways to "contradict" and to interrupt the internalization of negative stereotypes on all groups.

Creating opportunities for individuals (students, staff, and all groups) to express pride, build capacity, and receive recognition.

$\ulcorner$ Seek out and learn how other groups experience being in the school community and how you can be an ally to them; identifying where there is misinformation, a social injustice or mistreatment and correcting it.

Remember the painful effect of the stories of discrimination and increase your commitment to fighting the many "isms" that exist in the community; give individuals the opportunity to speak out about their experiences; determining the Speaks Outs that need to be told and heard in order to make the school environment safe and welcoming for all.

Identifying the most common situations in school where individuals experience prejudicial conduct and interrupt the behavior and/or support those affected.

$\ulcorner$ Other (please specify)

*3. Do you feel as though the training content helped you improve your ability to make decisions about the appropriate course of action involving prejudice behavior/conduct?
To a Great Extent
To a Considerable Degree
S Somewhat
$\checkmark$ Very Little
Not at All

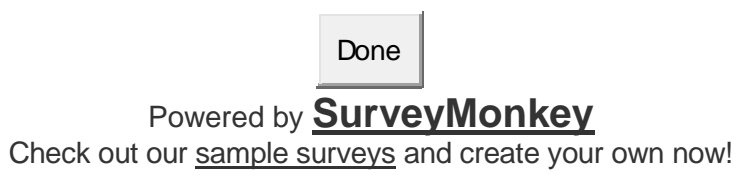




\section{Respondent \#1}

Q1: Which module(s) skills have you found useful in your work since you came back from training:

- Three Levels of Leadership

- Stereotyping: $1^{\text {st }}$ Thoughts Exercise

- Internalized Oppression

- Building Pride/Capacity Building

- Shifting Attitudes: Role Playing and Skits

Q2: Which areas of skills application have you shared with colleagues?

- Recognizing that in whatever roles we have within the school, we can take different levels of leadership to enhance the school environment for ourselves and others.

- Be willing to be aware of and re-examine our records about different groups and individuals that create tension and conflict and then get new information.

- Examine our own I.O. attitudes and behaviors in our school community. Make changes where needed by exploring ways to "contradict" and to interrupt the internalization of negative stereotypes on all groups.

- Creating opportunities for individuals (students, staff, and all groups) to express pride, build capacity, and receive recognition.

- Identifying the most common situations in school where individuals experience prejudicial conduct and interrupt the behavior and/or support those affected.

Q3: Do you feel as though the training content helped you improve your ability to make decisions about the appropriate course of action involving prejudice behavior/conduct? Response: To a Considerable Degree

\section{Respondent \#2}

Q1: Which module(s) skills have you found useful in your work since you came back from training:

- Three Levels of Leadership

- Stereotyping: $1^{\text {st }}$ Thoughts Exercise

- Internalized Oppression

Q2: Which areas of skills application have you shared with colleagues?

- Recognizing that in whatever roles we have within the school, we can take different levels of leadership to enhance the school environment for ourselves and others.

- Be willing to be aware of and re-examine our records about different groups and individuals that create tension and conflict and then get new information.

- Examine our own I.O. attitudes and behaviors in our school community. Make changes where needed by exploring ways to "contradict" and to interrupt the internalization of negative stereotypes on all groups.

- Other (please specify): Communication Skills

Q3: Do you feel as though the training content helped you improve your ability to make decisions about the appropriate course of action involving prejudice behavior/conduct? 
Response: To a Great Extent

\section{Respondent \#3}

Q1: Which module(s) skills have you found useful in your work since you came back from training:

- Stereotyping: 1st Thoughts Exercise

Q2: Which areas of skills application have you shared with colleagues?

- Help to create an environment that values and welcomes diversity; reach out to groups or individuals (Asking: "Did we leave anybody out?").

Q3: Do you feel as though the training content helped you improve your ability to make decisions about the appropriate course of action involving prejudice behavior/conduct? Response: To a Great Extent

\section{Respondent \#4}

Q1: Which module(s) skills have you found useful in your work since you came back from training:

- Three Levels of Leadership

- Stereotyping: $1^{\text {st }}$ Thoughts Exercise

- Making Commitments to Change: Speak Outs

Q2: Which areas of skills application have you shared with colleagues?

- Help to create an environment that values and welcomes diversity; reach out to groups or individuals (Asking: "Did we leave anybody out?").

- Be willing to be aware of and re-examine our records about different groups and individuals that create tension and conflict and then get new information.

- Identifying the most common situations in school where individuals experience prejudicial conduct and interrupt the behavior and/or support those affected.

Q3: Do you feel as though the training content helped you improve your ability to make decisions about the appropriate course of action involving prejudice behavior/conduct? Response: To a Great Extent

\section{Respondent \#5}

Q1: Which module(s) skills have you found useful in your work since you came back from training:

- Three Levels of Leadership

Q2: Which areas of skills application have you shared with colleagues?

- Be willing to be aware of and re-examine our records about different groups and individuals that create tension and conflict and then get new information.

Q3: Do you feel as though the training content helped you improve your ability to make decisions about the appropriate course of action involving prejudice behavior/conduct?

Response: To a Great Extent 


\section{Respondent \#6}

Q1: Which module(s) skills have you found useful in your work since you came back from training:

- [The respondent selected all eight modules' skills.]

Q2: Which areas of skills application have you shared with colleagues?

- [The respondent selected all eight module skills application.]

- Other (please specify): I shared with colleagues, open communication and engagement, staff relations and strategic planning.

Q3: Do you feel as though the training content helped you improve your ability to make decisions about the appropriate course of action involving prejudice behavior/conduct? Response: To a Great Extent

\section{Respondent \#7}

Q1: Which module(s) skills have you found useful in your work since you came back from training:

- Three Levels of Leadership

- Making Commitments to Change: Speak Outs

Q2: Which areas of skills application have you shared with colleagues?

- Recognizing that in whatever roles we have within the school, we can take different levels of leadership to enhance the school environment for ourselves and others.

- Creating opportunities for individuals (students, staff, and all groups) to express pride, build capacity, and receive recognition.

- Seek out and learn how other groups experience being in the school community and how you can be an ally to them; identifying where there is misinformation, a social injustice or mistreatment and correcting it.

Q3: Do you feel as though the training content helped you improve your ability to make decisions about the appropriate course of action involving prejudice behavior/conduct? Response: To a Great Extent

\section{Respondent \#8}

Q1: Which module(s) skills have you found useful in your work since you came back from training:

- Stereotyping: $1^{\text {st }}$ Thoughts Exercise

Q2: Which areas of skills application have you shared with colleagues?

- Recognizing that in whatever roles we have within the school, we can take different levels of leadership to enhance the school environment for ourselves and others.

- Help to create an environment that values and welcomes diversity; reach out to groups or individuals (Asking: "Did we leave anybody out?").

- Remember the painful effect of the stories of discrimination and increase your commitment to fighting the many "isms" that exist in the community; give individuals the opportunity to speak out about their experiences; determining the 
Speaks Outs that need to be told and heard in order to make the school environment safe and welcoming for all.

Q3: Do you feel as though the training content helped you improve your ability to make decisions about the appropriate course of action involving prejudice behavior/conduct?

Response: Somewhat

\section{Respondent \#9}

Q1: Which module(s) skills have you found useful in your work since you came back from training:

- Three Levels of Leadership

Q2: Which areas of skills application have you shared with colleagues?

- Help to create an environment that values and welcomes diversity; reach out to groups or individuals (Asking: "Did we leave anybody out?").

Q3: Do you feel as though the training content helped you improve your ability to make decisions about the appropriate course of action involving prejudice behavior/conduct? Response: To a Considerable Degree

\section{Respondent \#10}

Q1: Which module(s) skills have you found useful in your work since you came back from training:

- Environmental Scanning: Ups/Downs Activity

- Building Pride/Capacity Building

- Making Commitments to Change: Speak Outs Q2: Which areas of skills application have you shared with colleagues?

- Help to create an environment that values and welcomes diversity; reach out to groups or individuals (Asking: "Did we leave anybody out?").

- Be willing to be aware of and re-examine our records about different groups and individuals that create tension and conflict and then get new information.

- Remember the painful effect of the stories of discrimination and increase your commitment to fighting the many "isms" that exist in the community; give individuals the opportunity to speak out about their experiences; determining the Speaks Outs that need to be told and heard in order to make the school environment safe and welcoming for all.

Q3: Do you feel as though the training content helped you improve your ability to make decisions about the appropriate course of action involving prejudice behavior/conduct?

Response: Somewhat 